\title{
CASH2 - A MULTIPLE CATEGORIZATION FILE MANAGEMENT TOOL FOR UNIX/LINUX FILE SYSTEMS
}

\author{
By \\ Qunxiao Wang \\ A thesis submitted to \\ the Faculty of Graduate Studies and Research \\ in partial fulfillment of \\ the requirements for the degree of \\ Master of Science \\ Information Systems Science
}

Ottawa-Carleton Institute of Mathematics and Statistics

\author{
Carleton University \\ Ottawa, Ontario
}

April, 2006

(C) Copyright

2006, Qunxiao Wang 


$\begin{array}{ll}\begin{array}{l}\text { Library and } \\ \text { Archives Canada }\end{array} & \begin{array}{l}\text { Bibliothèque et } \\ \text { Archives Canada }\end{array} \\ \begin{array}{l}\text { Published Heritage } \\ \text { Branch }\end{array} & \begin{array}{l}\text { Direction du } \\ \text { Patrimoine de l'édition }\end{array} \\ \begin{array}{l}\text { 395 Wellington Street } \\ \text { Ottawa ON K1A ON4 }\end{array} & \begin{array}{l}\text { 395, rue Wellington } \\ \text { Ottawa ON K1A ON4 } \\ \text { Canada }\end{array}\end{array}$

Your file Votre référence ISBN: 978-0-494-16505-8 Our file Notre référence ISBN: 978-0-494-16505-8

NOTICE:

The author has granted a nonexclusive license allowing Library and Archives Canada to reproduce, publish, archive, preserve, conserve, communicate to the public by telecommunication or on the Internet, loan, distribute and sell theses worldwide, for commercial or noncommercial purposes, in microform, paper, electronic and/or any other formats.

The author retains copyright ownership and moral rights in this thesis. Neither the thesis nor substantial extracts from it may be printed or otherwise reproduced without the author's permission.
AVIS:

L'auteur a accordé une licence non exclusive permettant à la Bibliothèque et Archives Canada de reproduire, publier, archiver, sauvegarder, conserver, transmettre au public par télécommunication ou par l'Internet, prêter, distribuer et vendre des thèses partout dans le monde, à des fins commerciales ou autres, sur support microforme, papier, électronique et/ou autres formats.

L'auteur conserve la propriété du droit d'auteur et des droits moraux qui protège cette thèse. $\mathrm{Ni}$ la thèse ni des extraits substantiels de celle-ci ne doivent être imprimés ou autrement reproduits sans son autorisation.
In compliance with the Canadian

Privacy Act some supporting forms may have been removed from this thesis.

While these forms may be included in the document page count, their removal does not represent any loss of content from the thesis.
Conformément à la loi canadienne sur la protection de la vie privée, quelques formulaires secondaires ont été enlevés de cette thèse.

Bien que ces formulaires aient inclus dans la pagination, il n'y aura aucun contenu manquant.

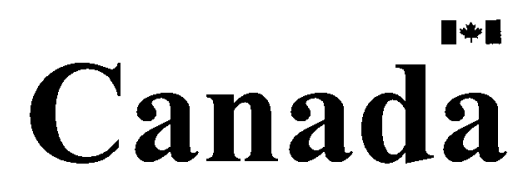




\section{Contents}

List of Figures

Abstract

Acknowledgments

1 INTRODUCTION

1.1 Motivation .......................... 4

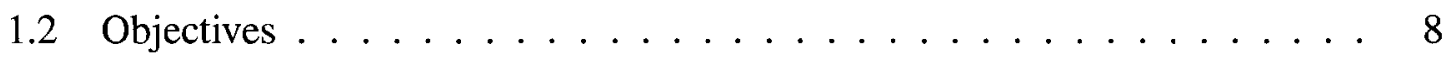

1.3 Outline of Thesis ..................... 10

2 BACKGROUND $\quad 11$

2.1 File Systems . . . . . . . . . . . . . . . . . 11

2.1.1 Files and Directories ................. 11

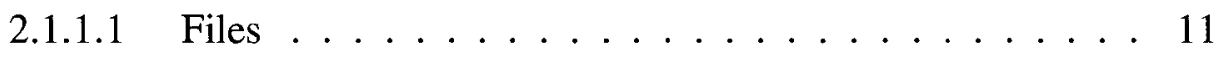

2.1.1.2 Directories ................ 13

2.1.1.3 Existing Problems ................ 14

2.1.2 Links in File System Operations . . . . . . . . . . . . . 16

2.1.2.1 Symbolic Links . . . . . . . . . . . . . . 16

2.1.2.2 Hard Links . . . . . . . . . . . . . . 17

2.1.2.3 Existing Problems .............. 17

iii 
2.2 A Study of Multiple Hierarchical Category . . . . . . . . . . . . . . . 19

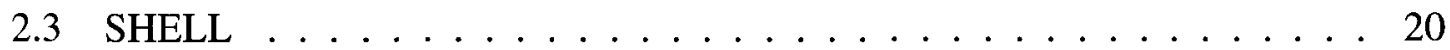

3 RELATED WORKS 22

3.1 Semantic File System . . . . . . . . . . . . . . . . . . . 22

3.1.1 MIT Semantic File System . . . . . . . . . . . . . . 22

3.1.2 Hierarchy And Content File System . . . . . . . . . . . . 26

3.2 Be File System . . . . . . . . . . . . . . . . . . 28

3.3 Desktop Search Tools . . . . . . . . . . . . . . . 30

3.4 Category-based Applications . . . . . . . . . . . . . . 31

3.4.1 Categories in Microsoft Outlook . . . . . . . . . . . . . . 31

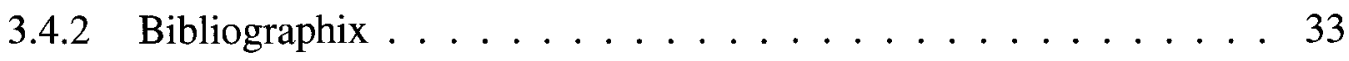

3.4.3 Automatic Categorization Tools . . . . . . . . . . . . . 33

4 BASIC CONCEPTS $\quad 37$

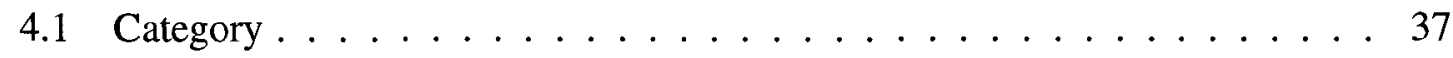

4.2 Multiple Categorization Structure . . . . . . . . . . . . 38

4.3 Hierarchical Categorization Structure . . . . . . . . . . . 39

4.4 Multiple Hierarchical Categorization Structure . . . . . . . . . . . . 41

4.5 Formalization of Multiple Hierarchical Categorization Structure of File Systems ............................ 46

4.6 Featured Properties . . . . . . . . . . . . . . . . 48

5 DESIGN OF THE CASH2 SYSTEM

5.1 CASH Design and Issues . . . . . . . . . . . . . . . 50

5.1 .1 Database Schema . . . . . . . . . . . . . 50

5.1 .2 Major Issues . . . . . . . . . . . . . . . . . 52

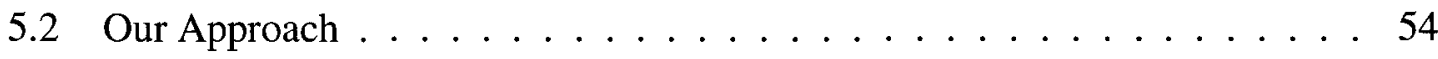


5.2 .1 System Architecture $\ldots \ldots \ldots \ldots 5$

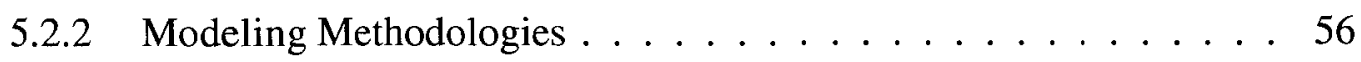

5.2 .3 A Study Of Database Engines . . . . . . . . . . . . . 57

5.2.3.1 Candidate Databases . . . . . . . . . . . . . 57

5.2.3.2 A Brief Introduction Of Berkeley DB . . . . . . . . . 61

5.2 .4 Database Design . . . . . . . . . . . . . . . . . . 64

5.2.4.1 Conceptual Database Design _ . . . . . . . . . 65

5.2.4.2 Logical Database Design . . . . . . . . . . 68

6 IMPLEMENTATION AND SYSTEM MEASUREMENT 72

6.1 Operations . . . . . . . . . . . . . . . . 72

$6.1 .1 \quad$ Basic Operations . . . . . . . . . . . . . . 72

6.1.1.1 Directed Cycle Detecting . . . . . . . . . . . 73

6.1.1.2 Path Computing . . . . . . . . . . . . . . . 74

6.1 .2 Category and File Operations . . . . . . . . . . . . 79

6.1.2.1 Category-based Search Operations . . . . . . . . . . 81

6.1.2.1.1 Command: 1scat. . . . . . . . . . 81

6.1.2.1.2 Command: cdcat . . . . . . . . . . 95

6.1.2.2 Add Operations . . . . . . . . . . . . . . . . 97

6.1.2.2.1 Command: mkcat . . . . . . . . . . . 97

6.1.2.2.2 Command: classify . . . . . . . . 100

6.1.2.2.3 Command: Incat . . . . . . . . . . 105

6.1.2.3 Remove Operation . . . . . . . . . . . . . . . 106

6.1.2.3.1 Command: rmcat . . . . . . . . . . 106

6.1.2.3.2 Command: decat. . . . . . . . . 116

6.1 .3 Other Operations . . . . . . . . . . . . . . . 120

6.1.3.1 Command: category and directory . . . . . . 120

V 


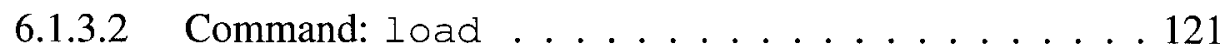

6.1.3.3 Command: pwdcat................ 121

6.2 Berkeley DB Implementations . . . . . . . . . . . . . . . 121

6.2.1 Using C Structures with Pointers for Database Record . . . . . . 121

6.2.2 Primary and Secondary Databases . . . . . . . . . . . 122

6.3 Solutions to Existing Problems in Directory Hierarchy . . . . . . . . . . . 124

6.4 Search-based Performance Comparison . . . . . . . . . . . . . 129

6.4.1 Experiment A: Directory/Category and File Search . . . . . . . 129

6.4.2 Experiment B: Directory/Category Path Search . . . . . . . . . 130

6.4.3 Experiment C: File Search . . . . . . . . . . . . 131

6.4.4 Experiment D: Category-based Search by lscat $-F \ldots 132$

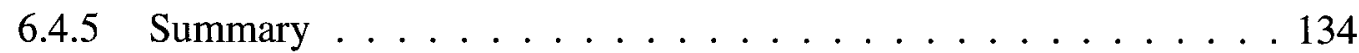

7 CONCLUSION REMARKS AND FUTURE WORK 135

8 APPENDIX: BRIEF DESCRIPTIONS OF USER COMMANDS 138

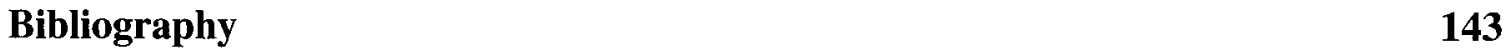




\section{List of Figures}

2.1 Inode and File Data . . . . . . . . . . . . . . . . . . 12

2.2 Example of Directory Entries . . . . . . . . . . . . . . . . 14

2.3 Examples of Directory Hierarchy . . . . . . . . . . . . . . . . . 14

2.4 Example of Links . . . . . . . . . . . . . . . . . . . 16

2.5 Examples of Directory Hierarchy $\ldots \ldots \ldots \ldots \ldots \ldots$

3.1 Sample Transducer Input and Output . . . . . . . . . . . . . . . 23

3.2 Multiple Categories in Microsoft Outlook . . . . . . . . . . . . 32

3.3 Multiple Categories in Bibliographix's Handling Project Interface . . . . . 34

4.1 Example of Multiple Categorization Structure . . . . . . . . . . . . . 39

4.2 Example of Multiple Hierarchical Categorization Structure . . . . . . . 43

4.3 Example of Category Names . . . . . . . . . . . . . . . . . . 45

5.1 Database Schema In CASH . . . . . . . . . . . . . . . . . . . . 51

5.2 Sample Category table and Category-Hierarchy table . . . . . . . 5 52

5.3 Graph Representations of Categories and Category-Hierarchy In CASH . . 53

5.4 System Architecture . . . . . . . . . . . . . . . . 55

5.5 Berkeley DB Subsystems . . . . . . . . . . . . . . . 63

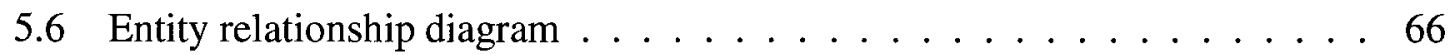

5.7 Database Schema . . . . . . . . . . . . . . . . . . . 69

vii 
5.8 Example of Tables $\ldots \ldots \ldots \ldots \ldots \ldots \ldots \ldots$

6.1 Algorithm of Directed Cycle Detecting . . . . . . . . . . . . . 73

6.2 Example: Directed Cycle . . . . . . . . . . . . . . . . . . . . . 74

6.3 Algorithm of Top-To-Bottom Path Computing . . . . . . . . . . 75

6.4 Example: Path Computing . . . . . . . . . . . . . . . . . 77

6.5 Algorithm of Bottom-To-Top Path Computing . . . . . . . . . . 78

6.6 Working Category and Aliases $\ldots \ldots \ldots \ldots$

6.7 Example: Sub-DAG Rooted at "Alcoholic" . . . . . . . . . . . . . . 85

6.8 Example: 1 scat $-i \ldots \ldots \ldots \ldots \ldots \ldots$

6.9 Example: cdcat . . . . . . . . . . . . . . . . 95

6.10 Add Category Operation . . . . . . . . . . . . . . . . . 98

6.11 Add Category Classification Operation . . . . . . . . . . . . . . 101

6.12 Add File Operation . . . . . . . . . . . . . . . . . . . . . . 103

6.13 Add File Classification Operation . . . . . . . . . . . . 105

6.14 Example: Incat . . . . . . . . . . . . . . . . . . . . . . 107

6.15 Remove File Operation . . . . . . . . . . . . . . . . . . . . . 109

6.16 Remove Category Operation . . . . . . . . . . . . . . 112

6.17 Example of rmcat $-k \ldots \ldots \ldots \ldots \ldots \ldots$

6.18 Remove File Classification Operation ～. . . . . . . . . . . . . . 117

6.19 Remove Category Classification Operation . . . . . . . . . . . . . . . 119

6.20 C Structures of Records in CASH2 Tables . . . . . . . . . . . . . 122

6.21 Example of Solution to Files with Lost Relationships . . . . . . . . . 126

6.22 Experiment A: Directory/Category and File Search . . . . . . . . . 130

6.23 Experiment B: Directory/Category Path Search . . . . . . . . . . 131

6.24 Experiment C: File Search . . . . . . . . . . . . . . . . . 132

6.25 Experiment D: Category-based Search by Iscat - F . . . . . . 133

viii 


\section{Abstract}

For the past several decades, the amount of information stored on personal computers has increased dramatically. How to handle this information efficiently in a file system becomes more and more challenging, and as a result, the focus of research has continued to shift towards better solutions for file organization and searching tools in personal computers. The most commonly used file organization structure in the file systems is the tree-based directory structure. It allows many-to-one classification of files into strictly hierarchical structures. However, files are more likely to have several different classifications, but this structure can only support a single classification. To overcome the drawbacks of this structure, CASH [13] was designed and implemented on top of the TC shell. It provides a category structure which allows a file to be classified to more than one category, and a category could also have multiple parent categories. However, due to modeling errors, CASH has some major issues left to be resolved with regards to its system design and implementation. It also has other drawbacks, such as lacking of formalized concepts, insufficient related work survey and back-end database engine studies, etc. In this thesis, a new system called CASH2 is designed and implemented as a file management tool for UNIX/Linux file systems. It is a significant extension of CASH. In CASH2, the data model has been re-designed and the command application has been re-implemented to correct the errors in the previous work. Moreover, the drawbacks of CASH have been overcome by formalizing the concepts of the multiple hierarchical categorization structure, surveying more of the related works, doing more study on database engines and choosing an engine that best 
fits the system requirements. In addition, more features has been added into CASH2, and a search-based performance comparison has been done between CASH2 and a UNIX file system. 


\section{Acknowledgments}

I could not have completed this thesis without the help of many people. I wish to thank:

Dr. Mengchi Liu, my supervisor, for showing me the right way to approach and deal with problems,

Dr. Yiqiang Zhao, for his kind guidance,

Dr. Shirley E. Mills, the chair of examining board, Dr. Brett Stevens and Dr. Samuel A. Ajila, members of the examining committee, for evaluating and discussing my work with me,

Ms. Valerie Daley, our Graduate Studies Administrator, for all her help,

Our lab-members, Lan Yang, Kelvin Gao and Heather Chen, for helping me with problems I encountered; Zhihong Li, for sharing her knowledge with me,

And most of all, my husband Dennis, my parents, and my sister Qunjie, for their love and support. 


\section{Chapter 1}

\section{INTRODUCTION}

\subsection{Motivation}

With the development of modern computer technology, storage capacities in personal computers has increased dramatically. Nowadays, storing multi-gigabytes of information in a personal computer is very common. Users generally do not worry about deleting old files to make room for storing new files. John R. Doucerur et al. [1] collected and analyzed about 10.5 TB of data from personal computers and found out that both the number of files and the file lifetime has largely increased during the past 20 years. When searching information within a small scale file system, users usually find it easier to find what they want. However, the more information users can store in a personal computer, with the longer times they will keep their files, the more challenges they will face in remembering what information they saved and where to find it. The focus of research nowadays is shifting towards better solutions for file organization and searching tools in a personal computer [2]. The most commonly used file organization structure in file systems is the directory hierarchy. It provides a mechanism to keep related files together in the directory-based tree structure. However, problems have resulted from this kind of organizational file structure. 
1. Tree structures only support single classifications and they lack the flexibility required for organizing a very large number of files: every directory can only have, at most, one parent directory, and every file can only be placed under one specific directory. As it is the nature of file organization, files are more likely to have several different classifications, but a directory hierarchy can only have a single classification for them.

2. To maintain file relationships users must place related files in the same sub-tree to avoid lost relationships. A simple example is the installation of a software package; typically executable, configuration, library and include files are stored in different directories, and by doing so, the relationship that those files actually belong to the same software package is lost. Users wishing to find, modify or delete these files may have problems as files are scattered and disorganized.

3. A directory hierarchy is a static organization scheme. When retrieving information, this static organization becomes a restriction. Users must remember the ordered sequence of directories they have used to organize the file when attempting to retrieve it by location, even though the subjects that users are interested in during retrieval might be different from those during organization [12].

4. People tend to forget the location of older files, and depend on searching tools to find them. In a directory hierarchy, users are bounded to traverse the whole directory tree structure. The result may not be accurate and there will be too much redundant search time, that spent only on walking the tree from root to the target for every search job.

In order to solve problem 1 we mentioned above, file systems have provided symbolic links and hard links as extended features to support organizing files and directories in more than one directory, however, some link related issues also aroused.

1. Hard links cannot cross file system boundaries, instead users have to use symbolic 
links.

2. Symbolic links are hard to maintain due to their weak connections with files. It is easy to have symbolic links "dangling" in the file system if the real files are deleted. This causes errors when users attempt to access them.

3. UNIX/Linux system provides one command $\mathrm{rm}$ for both physical file deletion and link deletions. The system will delete the file without warning if the link is the last pointer to it, even through users meant to delete a link and keep the file.

4. In some cases a search cannot be done by one tree traversal. Additional logical operations need to be applied to all the tree traversal results as well. There is no convenient way for users to work this out. Users have to learn to write a shell script, looping through each tree traversals while calculating the result.

5. Information about all the links of a given directory or file would help user to recategorize files and directories and also find correlated directories and files of interest within them. But due to the fact that the directory hierarchy can only provide topdown searches, and not the other way around, there is no convenient way to achieve this task. Users have to again write a shell script to get the result.

Now, there are indexing and search tools that help users find their files in the directory hierarchy. Tools such as find/grep and Glimpse [11] provide attributes-based searches for files based on full text analysis. Others, such as Google Desktop and Yahoo Desktop provide choices for users to gather attribute information for files with the desired file format (e.g., word document, pdf document, etc.) The advantage of these tools is that they speed up searches by applying indexes on file attributes. The disadvantage is that their search functions are conditional on the data format they support, which leaves large number of files without being indexed and out of the reach from these tools. In addition, users cannot classify files themselves according to their own taste. Also, due to the nature of the full 
text indexing, attributes with little semantic meanings are indexed along with those with semantic meanings, which leads to large storage usage, e.g., Google desktop requires 500 megabytes of disk space for indexing [30]. Moreover, all these tools do not provide a mechanism to recognize relationships between individual file attributes. Another important fact about full-text searching is that, it can serve as a complement for directory (or category, described in details in Chapter 4) hierarchy, but the search alone may not solve most of the retrieval problems due to the fact that it is of little use if users are not able to recall the precise details of the target file they are looking for [12].

Lansdale suggested that retrieving information involves two distinct psychological processes: recall-directed search, followed by recognition-based scanning [9]. Recall-directed search means that by memorization, users get as close as possible to files they are looking for, such as remembering file names, or their locations in a directory hierarchy. Recognitionbased scanning means that when specific recall has failed, then the memory might identify an area of the possible locations of files, as a result, recognition-based scanning within that area is required. Human-assigned directories and categories serve as guides in the process of recognition-based scanning [12].

Several groups have also developed new file systems that offer indexing-based search for the files, such as the Semantic File System [23], the Hierarchy And Content file system (HAC) [25], the Be File System (BeFS) [24], and so forth. By assigning multiple attributes to a single file, the information available to the search tools in these systems is largely increased. Unfortunately, similar to the indexing and the search tools we mentioned above, some of these systems "make no limitations on the methods for gathering attributes" [2], and some of them depend on full text content analysis to provide attribute information, making them not much different from existing indexing and searching tools.

A large number of automatic categorization tools, such Verity's Intelligent Classifier [31], Inxight's Categorizer [33], and Mohomine's Mohn Classifier [34], have been developed in the past few years. Most of these tools focus on automatic tagging on documents with 
metadata, automatic assigning documents into categories, and helping in revealing the taxonomy of a set of documents, etc. One common characteristic among all the automatic categorization tools is that, more or less, they all require sample documents to get their engine trained before they start the automation work. Following training, reviewing and tuning is needed at regular intervals to maintain (or increase) the accuracy rate. No automatic categorization tools are $100 \%$ automatic [37]. In reality, they are great aids in making the human-assigned categorization and indexing task more feasible, as human decision are still the dominant part for the categorization work.

As an attempt to solve the problems in the existing directory hierarchy, CASH [13] was designed and implemented on top of the TC shell. It provides a category structure which allows a file to be classified to more than one category, and a category could also have multiple parent categories. However, due to modeling errors, CASH has some major issues left to be resolved with regards to its system design and implementation. It also has other drawbacks, such as lacking of formalized concepts, insufficient related work survey and back-end database engine studies, and so forth. We will address in more detail the problems in CASH in Chapter 5.

\subsection{Objectives}

The primary goal of our work presented in this thesis is to design and develop CASH2 a file management tool for UNIX/Linux systems based on a multiple hierarchical categorization structure, which overcomes the drawbacks of the existing directory hierarchy and provides more flexible ways for users to organize, manage, maintain and search for files in the file system. In CASH2, we improve the work based on CASH, and make significant progresses on CASH. Below we enumerate a list of our works, which will serve as the objectives of this thesis as well.

1. The previous work on CASH has insufficient study on related industrial and research 
work. In $\mathrm{CASH} 2$, we should study the existing solutions and perform a survey of related work in order to improve our research.

2. Some basic concepts in CASH were not defined accurately, e.g., "category", "multiple hierarchical categorization structure", and so forth. We will correct them in $\mathrm{CASH} 2$.

3. The previous work on CASH explained its features to provide better operations than links to organize files in UNIX/Linux file systems. There are more issues in the directory hierarchy, and CASH is able to provide better features as solutions. However, the previous work did not explain clearly what these features are. In CASH2, we will do more work around this aspect.

4. CASH proposed a database model to realize its multiple hierarchical categorization structure. However, it did not explain the modeling methodology and it was poorly modeled and designed, In CASH2, we will do more work around this aspect.

5. MySQL has been chosen as the back end storage engine to realize the database model in CASH. However, the previous work on CASH has no study on comparing different database engines and choosing one that fits the applications of CASH most. In this thesis, we will study different database engines and make our choice according to the features of $\mathrm{CASH} 2$.

6. Due to the design errors, CASH did not provide correct operations and algorithms to realize its features. In CASH2, we will improve the work in this respect.

7. In CASH, the command design has omitted some nice features of category management on files, e.g., files classified to a child category should have been found when users issue a search command for files classified to its parent category, and there is no command to show all the paths of a given category (as to solve the problem 5 of directory hierarchy on page 18). We should enhance our work in this aspect. 
8. The previous work on CASH did not provide evaluations to measure itself. In $\mathrm{CASH} 2$, we will add more in this aspect.

\subsection{Outline of Thesis}

The thesis is organized in four parts. The first part introduces background knowledge concerned in this thesis. The second part describes the related work and existing solutions. The third part describes the modeling and design of the prototype system. The fourth part describes the implementation of experimental system. The thesis ends with a summary and conclusion, and poses future research questions. The content of the chapters, is described in more detail as follows:

- Chapter 2 introduces important background knowledge for understanding this thesis.

- Chapter 3 gives a survey of related work and existing solutions.

- Chapter 4 describes the basic concepts in CASH2.

- Chapter 5 presents the design of CASH2.

- Chapter 6 discusses implementation details of CASH2. It explains operation rules by showing an running example throughout this chapter. It also presents search-based performance comparisons between $\mathrm{CASH} 2$ and UNIX/Linux directory hierarchy.

- Chapter 6 summarizes and concludes with future research options.

- Appendix presents descriptions of user commands. 


\section{Chapter 2}

\section{BACKGROUND}

\subsection{File Systems}

The main purpose of computers is to create, store, modify, save and retrieve data. A file system provides support for these objectives. Technically speaking, a file system [3] is the set of system software that contains methods and data structures that an operating system employs to track files. At the highest level, closest to the user, the file system provides a way to organize, store, retrieve, and manage information on a storage media such as a raw disk or a partition. At a lower level, the file system manages permanent storage and is considered part of the operating system.

\subsubsection{Files and Directories}

There are two fundamental concepts in a file system: files and directories.

\subsubsection{Files}

A file $[4,24]$ is a piece of information that is stored in a computer system and it can be identified and referenced later on by the name given to it. The piece of information can be 
a bit of text, (e.g., a note, a script, a letter, etc.), a graphic image, a video stream, a database, or any collection of bytes users wish to store permanently. The file system also allows users to name the file in a meaningful way. The size of a file can range from a few bytes to the entire capacity of a partition or disk. The file system should be able to contain a very large number of files.

The system information for a file is called metadata [5, 24]. Each file is associated with a metadata structure, stored with file attributes, such as physical address on a disk, user and group ownership, access mode (read, write, execute permissions), last access or last modification time, creation time, size, type and so forth. The UNIX/Linux file systems maintain a data structure called index node (inode) $[3,4,5,24]$ to store the metadata of a file. Inodes build up the connection to the actual data in the file. Figure 2.1 is a simple example that shows how to map a file data using inode. The basic idea is that when users request data in a file, the file system will map it to a physical disk location using the system information stored in inode, request the related block(s) from the disk, and then pass the data back to the users.

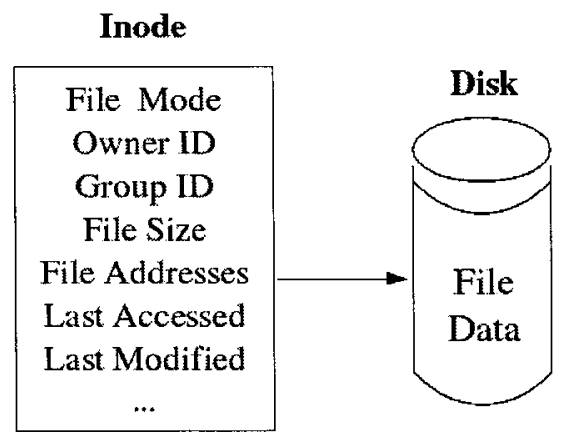

Figure 2.1: Inode and File Data 


\subsubsection{Directories}

Above we explained a file as a unit of storage for users data. A file system must provide means to name and organize multiple files. Therefore, it introduces a concept of a directory (also known as folder) [3, 5, 24]. In UNIX/Linux file systems, a directory is a file, which has an inode information associated with it. The directory file has special write protection privileges so that only the file system can write into it, while read access is available to user programs [3]. Therefore, users can use the physical disk location stored in the directory inode to access the directory file data, which contains a list of directory entry. A directory entry consists of a file name and a pointer to associated inode (i.e., index number). Figure 2.2 gives a conceptual picture of directory entries. There are three entries under the current directory: a file name "red" with its index number "293", "yellow" with " 587 ", and "white" with "876" (in this example, these names are real file names, and not in the directory manner.) The main purpose of a directory is to map files with their names in the directory to the associated files by means of index numbers. The file name is the key on which the directory searches when looking for a file. In Figure 2.2, when users attempt to open a particular file with the name "red", the file system must search the directory entries to find the requested name. If the name "red" exists, the file system uses the associated index number " 293 " to locate the metadata about the file (i.e., the inode of the file), then it loads that information and allow access to the file data (as shown in Figure 2.1). If the name "red" does not exist, the file system will return an error such as "No such file or directory".

File systems provide different techniques to organize directory entries. The simplest approach, is to store each entry sequentially, and a linear scan has to be used for a file name lookup. However, when directories contain a very large number of entries, such an organization becomes inefficient. In those cases, a B-tree, or hashed structure is preferred $[3,5,24]$.

A single directory is not sufficient for structuring collections of files. The universally 


\section{Directory Entries}

\begin{tabular}{|c|c|}
\hline \multicolumn{2}{|c|}{ File Name } \\
\hline red & 293 \\
\hline yellow & 587 \\
\hline white & 876 \\
\hline
\end{tabular}

Figure 2.2: Example of Directory Entries

adopted approach is the hierarchical, or, a tree-structure approach [3]. The simplest implementation is to allow an entry in a directory to contain a name that refers to another directory. A directory inside another directory is known as a subdirectory [3]. Figure 2.3 gives several examples showing how a directory hierarchy may look like. In case (a), a single file "foo" is at the top level. Case (b), (c) and (d) shows different combinations of directories and files in the hierarchical structure.

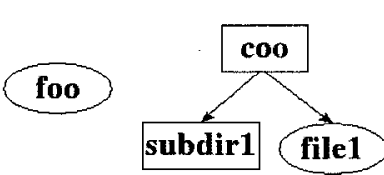

(a) (b)

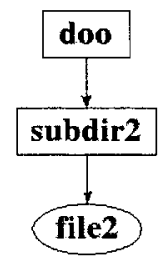

(c)

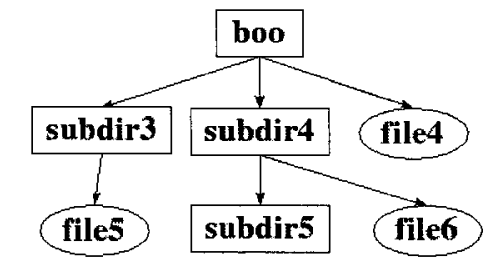

(d)

$\square$ : Directory $\longrightarrow$ : File

Figure 2.3: Examples of Directory Hierarchy

\subsubsection{Existing Problems}

Files and directories are two basic abstractions of the data structure of the file system [24]. A directory hierarchy is helpful to organize files by keeping related files together. However, 
the nature of tree structure does not provide flexibility required for organizing a very large number of files. The following outlines some of the drawbacks:

1. Every directory other than the root directory can only have at most one parent directory, and every file can only be placed under one directory. However, As it is the nature of file organization, files are more likely to have several different classifications, but the directory hierarchy can only have a single classification for them.

2. Files have to be placed under the same directory, otherwise, their relationship is lost. For example, when installing software packages into a UNIX/Linux machine, the conventional rule is to store executable, configuration, library and include files into different directories. Since these files are not kept under the same sub-tree anymore, the relationship that they actually belong to the same software package is lost.

3. Using the directory hierarchy, users are bounded to a static organization schema. While retrieving information, the static organization becomes a restriction. In particular, users must remember the ordered sequence of directories they used to organize the file when attempting to retrieve it by location, even though "the topics of interest during retrieval might be different from those during organization" [12]. For example, users are interested in food recipes, so they organized some Asian food recipes under directory path "/ Food/ Asian/". Later on they changed their topic of interest to "Asian-related subjects", as a result, they may try to retrieve those saved recipes by a path like "/ Asian". Obviously, they will have problems finding them since the retrieving directory path is not the same as the path under which those files were originally organized.

4. If users forget the file location, e.g., as we just discussed in the previous example, if users forget where they stored their food recipes, they then have to traverse the whole directory hierarchy to find it. The result may not be accurate, and there is too 
much redundant search time, spent only on walking the tree from root to the target for every search job.

\subsubsection{Links in File System Operations}

In order to overcome the limitation of the directory hierarchy we addressed above in problem 1 , the file system has provided an extended feature, known as links in UNIX/Linux (short cuts in Windows and alias in Mac OS).

Basically, there are two types of links in a file system: symbolic links and hard links.

\subsubsection{Symbolic Links}

A symbolic link $[3,10,24]$ is a named entity in the file system that simply refers to another file (or directory). In other words, a symbolic link is a named entity in a directory with the associated index number referring to a file. The data of the file contains only a piece of string - the name of another file (or directory). For example, in Figure 2.4, a directory contains a symbolic link named paper, and this symbolic link refers to a file called publication in the same directory. Whenever a program opens the file paper, the file system will transparently open the target of the link, which is the publication file.

\section{Directory Entries}

\begin{tabular}{|c|c|c|}
\hline Inode Table & \multicolumn{3}{c}{ Index Number } \\
\hline 293 & publication & Original File \\
\hline $2 . .$. & myPublication & Hard Link \\
\hline 293 & paper & Symbolic Link \\
\hline 579 &
\end{tabular}

Figure 2.4: Example of Links 


\subsubsection{Hard Links}

A hard link $[10,24]$ is also a named entity in the file system. It does not have an inode of its own. Instead, it contains the index number of another file as shown in Figure 2.4. A hard link has a very strong connection with the file, even under the circumstance that the original file is moved or renamed, the hard link still remains the same. A hard link is preferred when a connection to a file must not be broken.

\subsubsection{Existing Problems}

The File system provides symbolic links and hard links as extended features to support organizing files and directories in more than one directory. However, some issues still remain as follows:

1. Hard links cannot cross file system boundaries, which limits its usage. Users have to use symbolic links instead if they wish to link files in different file systems.

2. Because a symbolic link is a data file that contains the name of the file it is linked to, the connection between them is weak. Under the circumstance that the linked file is moved, renamed or deleted, the symbolic link would be left "dangling" and does not work properly anymore. In other words, symbolic links are poorly managed unless users manually maintain them.

3. Command $\mathrm{rm}$ in UNIX/Linux file systems is a removing utility for both physical files and links. When users issue a rm command to remove a link, they have to make sure that the given link is not the last pointer to a file. Otherwise, the system will delete the file, even through the users meant to delete a link and keep the file.

4. If a search requires more than one tree traversal, and additional logical operations to be conducted on all the tree traversal results, the file system does not provide direct and convenient utilities to fulfill this request. For example, as shown in Figure 2.5, 
the file "f1" is located under "/ a/ b/ e", and has two hard links under "/ a/ c/ g", and "/ a/ d/ j". A request such as " find files that under directory e, $g$ and $j$ at the same time" requires three tree traversals to search for location "/ a/ b/ e", "/ a/ c/ g", and “/ a/ d/ j", and a logical AND operation on all three tree traversal results. To conduct this search, users must write a scripting program, looping through each tree traversals while calculating the result.

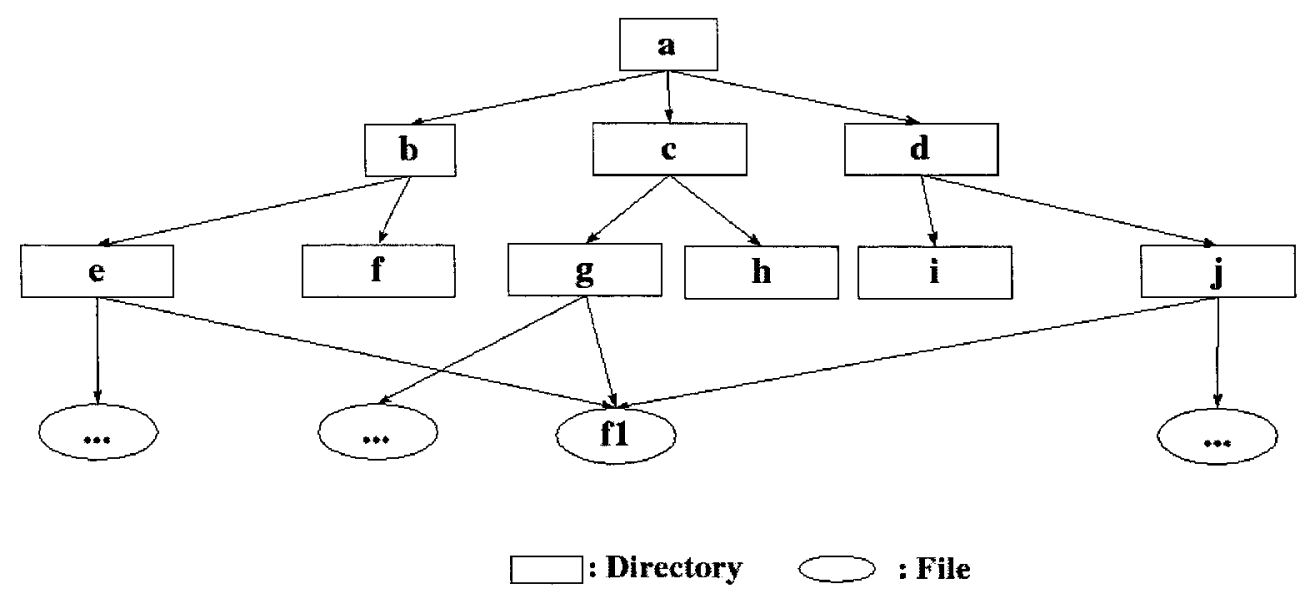

Figure 2.5: Examples of Directory Hierarchy

5. All of the link information about a file would help users to re-categorize files (or directories), or find correlated directories and files of interest within them. For example, in Figure 2.5, if users were aware that the real location of the file " $\mathrm{fl}$ " is under "/ a/ b/e", and at the same time were provided with all its links information, " a/ c/ g", and "/ a/ d/ j", it is easier for them to decide if they wish to update the link information, or to further discover files under directories that showed up in the link path, such as "c" and " $\mathrm{d}$ ". However, the conventional implementation of directory hierarchy only provides the unidirectional search (i.e., root to leaves search), not the bidirectional search (i.e., both root to leaves and leaves to root searches). Therefore, there is no convenient way to locate and update all link information of a file when 
a user attempts to re-categorize directories (or files). Users again, have to write a scripting program, and apply different tree traversals to get the result.

\subsection{A Study of Multiple Hierarchical Category}

MIT AI Laboratory carried out a user study in 2003 to examine the usefulness of humanassigned multiple categorization as opposed to directory storage containment [12]. Their objective was to "examine multiple categorization as a useful technique, and an alternative organization and navigation mechanism for commonly-used forms of information". In particular, they explored the user interface consequences of multiple categorization support. They adopted a category-based organization and navigation schema that allows information to be placed simultaneously in a semantic category hierarchy. At the same time, users were provided with folder hierarchy for comparison. The participants in this study were twenty one graduate students in MIT computer science department. The test environment was a modified Internet Explorer window with an organization/navigation panel, a content pane for browsing a web page, and an instruction pane prompting users with options of either classifying a page or answering a question. The experiment was divided into two sessions: organization session and navigation session. In the organization session, participants were asked to organize two corpora of information using two different techniques we mentioned above. A week later in the navigation session, participants were asked to answer two sets of questions by using two different navigation techniques corresponding to the organization structures. The result of the organization session showed that users took $19 \%$ less time organizing their corpus using multiple categorization compared to folders. The result from answers of questions showed that among all the participants, $38 \%$ felt that multiple categorization more closely matched the way they think about the information, $52 \%$ felt both structures captured how they modeled information. Participants have also created twice as many categories as folders, showing their great interest towards human-assigned categories. 
The result of the navigation session showed that multiple categorization method had a $17 \%$ improvement on average retrieving time comparing to folder method. Participants also felt that when the information became correlated and overlapped, multiple classification was very helpful, and classifying documents into a hierarchy reduced the scanning needed to locate relevant categories.

Their findings are a great support to our research of providing a multiple hierarchical categorization file management tool.

\subsection{SHELL}

The shell is a special program that essentially provides a kind of interface between users and the innards of the UNIX/Linux operating system (a program called the kernel). When the system boots up, the kernel is loaded into memory to manage the system until the system shuts down. It creates and controls processes, and manages memory, file systems, and so forth. The shell program resides on the disk, and the kernel loads it along with other programs such as shell scripts, etc., into memory, executes them, and cleans up the system when they terminate. The shell is a utility program that allows users to interact with the kernel by interpreting commands that are typed either at the command line or in a script file [22].

\section{TC Shell}

There are several prominent and supported shells on most UNIX/Linux systems: the $C$ shell (Berkeley shell), the Bourne shell (AT\&T shell), the Korn shell (a superset of the Bourne shell), and bash shell (an enhanced Bourne shell). There are slight variations between different versions of the shells, e.g., some differences in command syntax. However, in most cases, all these shells behave almost the same way when running interactively. The $\mathrm{C}$ shell (csh) and TC shell (tcsh) provide a number of convenient features for interactive use that 
are not available with the Bourne shell, such as filename completion, command aliasing, history substitution, job control, and so forth. When used as a programming language, these shells are almost identical. However, the TC shell has many more interactive features than its predecessor, the $\mathrm{C}$ shell. TC shell is included in most UNIX/Linux distributions, Windows, and more. There are a number of new features in the TC shell, such as shortcuts, command-line editing, programmable completions (filenames, commands, and variables), spelling correction, and so forth [22]. 


\section{Chapter 3}

\section{RELATED WORKS}

In objective (1) (on page 8) of this thesis, we point out that the previous work of CASH has insufficient study on related industrial and research work. We now discuss this in more detail around related works in semantic file systems, indexing and search tools, and category-based search applications.

\subsection{Semantic File System}

Due to the limitation of the traditional file systems, several research groups have developed semantic file systems to offer index-based searches for files. Here, we describe a couple of them.

\subsubsection{MIT Semantic File System}

The Semantic File System was developed by an MIT research group (MIT SFS) [23]. Firstly, it introduces the concept of associative access which means that the programs, documents, and other relevant objects in the system can be located according to their attributes, such as its author, size, type, name, and so forth. Secondly, this system introduces 
the transducer program to extract attributes with their values from the files in the file systems. Figure 3.1 is taken from [23] to illustrate an example of transducer input and output. There are different transducers for different types of files in the file system, and MIT SFS has some default transducers that can process most of files in the file system. Optionally, users may also choose to write their own transducers for their personal files. The output of transducers are passed to an indexer database in a form of (attribute, value) pairs. The indexer database does a fast attribute lookup when a query is issued. The transducers are able to utilize the information about the semantics of the updated or newly created objects in the file system to extract the attributes for indexing.

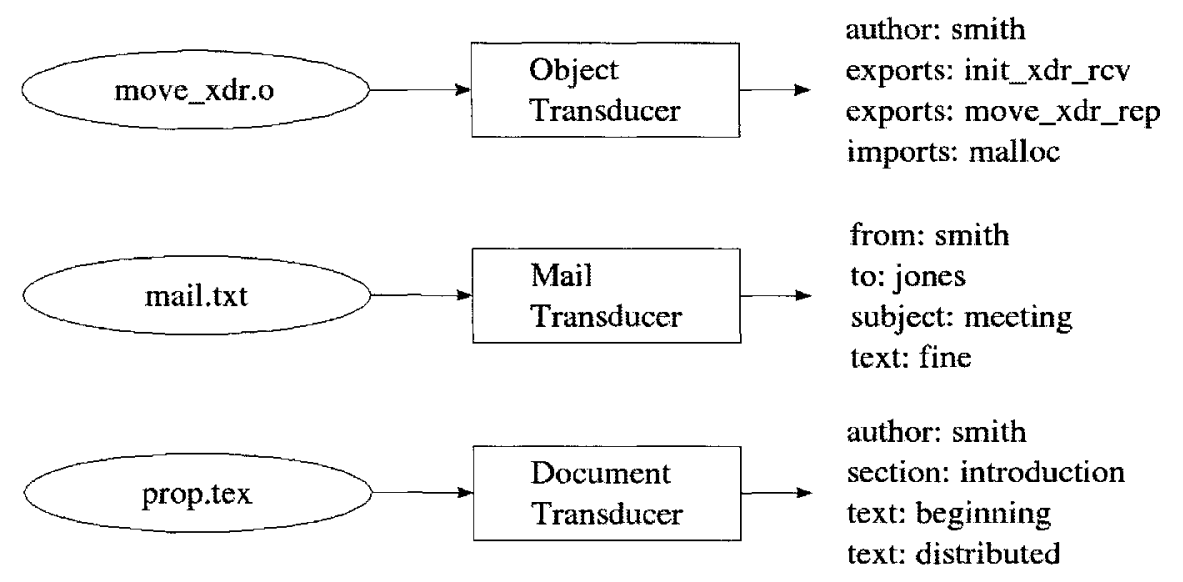

Figure 3.1: Sample Transducer Input and Output

The query in MIT SFS is defined as a virtual directory. The virtual directory names are used as conditions for queries. The content of a virtual directory is a set of symbolic links to files in the physical file system that satisfies the query that the virtual directory represents. The difference between a virtual directory and a physical directory is that users do not need to create a virtual directory to access the contents of it. A virtual directory is computed on demand. MIT SFS maintains a cache to map virtual-directory names to the 
results of queries, and keep the cache updated by periodically re-indexing the files and reevaluating the queries of the virtual directories in its cache. In MIT SFS, users can execute command $\mathrm{cd}$ to get into a virtual directory, and examine the result of the query by issuing the ls command "within" the query, or, they can simply execute ls followed by a virtual directory to get the result as well. The following examples show how the system works.

$\therefore \mathrm{cd} / \mathrm{sfs} / \mathrm{author:}$

Command cd followed by the virtual directory author is meant to locate all authors that have files in the sfs system.
$\therefore$ is $-\mathrm{F}$
\% Leed Joned Smith@

Command is $-F$ is meant to display the result returned by the previous query. The following line is the result of the query, which shows that in the system, we have three authors, "Lee", "Jone" and "Smith".

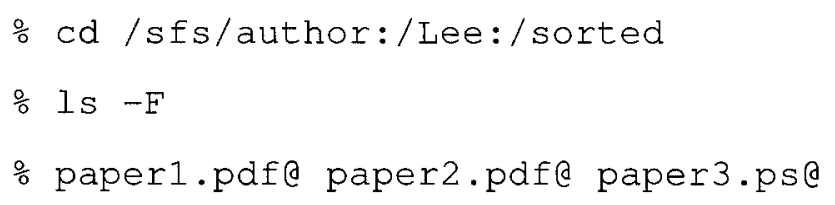

Command $\mathrm{cd}$ followed by the virtual directory author:/Lee:/sorted is meant to locate all the files that export the procedure sorted, and then further restrict this set of files to those with their author named "Lee".

Command ls $-F$ is meant to display the result of a set of symbolic links to files that satisfies the previous query.

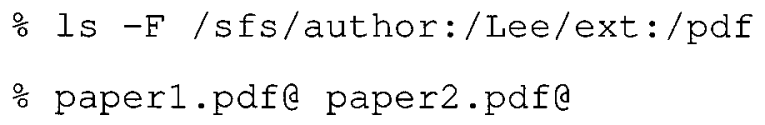


Command Is $-E$ followed by the virtual directories / sfs/author: / Lee/ext:/pdf is meant to display the query result of all the files with "Lee" as the author and having the file extension "pdf". The following line show the result set of symbolic links to files that satisfy the conditions.

To sum up, MIT SFS provides an associative attribute-based access to the content of an information storage system with the help of file type specific transducers. It also integrated this access into the file system with virtual directories. It is fast enough to be usable and provides an enhanced search ability, allowing people to locate files they would otherwise have had difficulty finding. It is an effective solution to the problems of content-based file access. Furthermore, such a file system can effectively be layered on top of a traditional hierarchical UNIX/Linux file system by applying overloading of symbols and virtual directories.

Although MIT SFS has some novel features, it also has some drawbacks. First, MIT SFS relies on a flat structure (i.e., multiple virtual directories). Although a file can belong to several virtual directories, there is no virtual directory hierarchy exists in the system. As a result, uses are not able to utilize the relationship between different virtual directories to refine query result. Second, MIT SFS only allows uses to use virtual directories to organize results of queries, but use the underlying file system to organize files. Third, virtual directories are extension-based, which means the content of queries, which is a list of semantic links to the physical files returned from the queries, determines the meaning of virtual directories. However, query results depend on what files that the underlying transducers have indexed. If the system does not have the specific transducer for the specific file, then the files will not be indexed and the query result will not be complete. Additionally, the result may not be accurate as transducers can make mistakes thereby classifying files wrongly. Fourth, the virtual directories are in the form of (attribute: value) pairs or the conjunctions. The system assumes that queries are logical AND combinations of (attribute, value) pairs which means that the queries cannot have logical operations other than AND on all the sets 
of results. Fifth, MIT SFS does not allow users to manually tune the query result by adding or removing files to or from the query result of a virtual directory, therefore, users do not have the freedom of modifying the result according to their own wishes.

\subsubsection{Hierarchy And Content File System}

The Hierarchy And Content file system (HAC) [25] is also a semantic file system. It integrates content-based access mechanisms with hierarchical file systems. It has some similar design concepts compared to MIT SFS, and improved over MIT SFS in many ways.

Firstly, instead of using file type specific transducers, HAC uses Glimpse [11] to provide content based access to files in the underlying file systems. Glimpse is a search tool that provides indexing and query schema for file systems, it extends agrep, a fast approximate pattern-matching tool, to the entire file systems. Glimpse provides full-text retrieval, including logical queries (AND, OR), and approximate matching [11].

Secondly, HAC maps queries into directories. Such directories are called semantic directories. When users create a new semantic directory, they issue a command smkdir, and specify both its path name and its query. Then, HAC creates a new directory, and associates it with the query. In the meantime, HAC contacts the underlying index database to evaluate the query. In the new directory, HAC automatically creates new symbolic links to all files that satisfy the query, this set of pointers is called the query-result of the directory. In the meantime, these symbolic links can co-exist with other information in the semantic directory, including other symbolic links or other regular files. The symbolic links can also point to files in other semantic directories in the file system, or to remote file systems. Semantic directories provide the abstraction and utility of virtual directories, but in HAC they are also regular hierarchical directories. Users can add files to them, modify them and apply other applications on them.

Thirdly, users can also create subdirectories within semantic directories, so that they 
can organize their files in a hierarchy and access them by specifying path names, and at the same time, retrieve files by asking queries that describe the conditions. This enables query-refinement and customization. When a user creates a semantic sub-directory within a semantic directory, the file system ensures that the query-result of the sub-directory is a subset of the query-result of its parent. As a result, users can create a hierarchy of semantic directories to refine their queries. Users can also modify the query-result by editing the set of pointers in a semantic directory, without modifying its query or the files in the file system. In this way, users can customize the results of queries according to their own personal tastes.

To sum up, HAC is a semantic file system that enables both name-based and contentbased access to files. Users can create their own name spaces based on queries, on explicit path names, or on any combination of the two. HAC also enables query-refinement, so that it makes it faster, easier, and more convenient for users to find their file of interest. Additionally, comparing to MIT SFS, the queries in HAC can have logical operations AND and $\mathrm{OR}$, on all the sets of results, and it allows users to manually tune the query result by adding or removing files to or from the query result of a virtual directory, therefore, users have the freedom of modifying the result according to their own wishes.

Although HAC have some novel features, some of the drawbacks still exist. Firstly, the semantic directory in HAC is still extension-based, which means it is dependent upon the list of symbolic links returned from its associated query. Hence, the content of the query-result determines the meaning of the semantic directory. When users create such a directory, the system returns information based on what Glimpse has indexed, It causes a bottle neck that limits the completeness and accuracy of the query result. Secondly, the full-text indexing functionality enabled by Glimpse search engine indexes information both with and without semantic meanings, this leads to a large storage redundancy. Thirdly, while mapping virtual directories to files, HAC indexes a full path of virtual directories, which also causes redundancy and inefficiency in the data storage. 


\subsection{Be File System}

The Be File System (BeFS) [24] is a native file system that supports database concepts in the file system. There are three features that contribute into the database functionalities in BeFS, Attributes, Indexing, and Queries.

\section{Attributes}

In BeFS, an attribute is a name and a data value associated with it. An example is as follows:

keywords = paper, publication

The data associated with an attribute name is free-form and can be anything. In the design of inode structure in BeFS, a field named attributes is added. This field is an inode address, and the inode it points to is a directory that contains attributes about this file. By doing so, attributes are attached to files and information about the contents of the file is stored. BeFS also provides straight forward attribute API for users to perform different operations on attributes, such as write, read, remove, etc.

\section{Indexing}

BeFS maintains indexes of attribute values. Users can create indexes if they wish to run queries about a particular attributes. For example, the mail service creates indexes named From, To and Subject to the fields of an email message. Then, for each arriving message (in BeFS, messages are stored in individual files), the mail service adds attributes to the file for the From, To and Subject fields of the message. Then, BeFS indexes the value for each of the attributes. Assume a piece of email arrives with From fields filled with abc@yahoo.com, the mail service in BeFS adds an attribute whose name is From and whose value is abceyahoo.com to the file of this message. BeFS then checks that the attribute name From is indexed, and so it adds the value of that attribute, i.e. abc@yahoo and the inode address of 
the file to the From index. BeFS also enables automatic B-tree indexing of three file attributes: file name, file size and the last modification time. These three indexes apply to all the files in the system. so that it is easier to issue query like "size greater than 1 GB" without going through all the files in the system to decide which one matches. BeFS uses B+trees to maintain theses indexes, and allows an arbitrary number of indexes exists in the system, so that users are free to create any index according to their wishes.

\section{Queries}

The purpose of indexing in file systems is to bring convenience for users to issue queries that use the indexes to efficiently obtain the result. Indexing speeds up searches significantly comparing to walking directory trees and examining each file in an ordinary file system. In BeFS, a query in BeFS is a string that contains an expression about file attributes. The query language is built up with expressions joined with logical AND or logical OR connectives. The following example shows how the queries work.

$$
\text { name }==\star \text {.java \&\& size }>12000
$$

This query line is meant to find all the java files in the system with the file size bigger than 12000 byte.

MAIL:status $==$ Old \&\& MAIL: send_to $!=$ mailinglist@yahoo.com

This query line is meant to display old emails that has been sent to email addresses that are not in the mailing list.

$$
\text { type }==\text { pdf }|| \text { type }==\text { ps }
$$

This query line is meant to find all the pdf files and ps files in the system.

Once the query engine determines that a file matches a query, the inode information 
of the file is returned. The process of converting the query result into readable information for users requires the file system to convert an inode into a file name. In normal file systems, this would not be possible, however, BeFS makes it happen by designing its inode structure differently. Besides the ordinary fields that an inode structure normally includes as we mentioned in section 2.1.1.1, BeFS adds two more fields: parent and small_data. The field parent is a reference back to the directory that this file is located in. The field small_data is used to gain efficient access to a reasonable number of small attributes. In this field, BeFS stores the name of the file this inode refers to, together with the parent field, BeFS can reconstruct the full path name of a file given just the inode.

To sum up, BeFS supports file attributes (name/value pairs) indexing, which enables query functionalities to locate files in a fast and convenient way. However, the attribute indexing method forms a multiple categorization structure (i.e., one level flat categorization structure). This type of structure has two major drawbacks, if categories tend to be finegrained, then the number of categories may grow very large, or, if the categories are too coarse-grained, the number of files classified to a certain category can grow very large as well. In both cases, users will have difficulties locate their target files. Moreover, BeFS does not provide a hierarchical structure which maps the relationship between all of these attributes to refine file retrieval.

\subsection{Desktop Search Tools}

Desktop search tools, such as Google Desktop [27], Yahoo Desktop [28], and MSN Desktop [29], have come into existence in the last several years. They provide users with easy access to information on their computers and from the web by enabling full-text search over email, files on disk, music, photos, chats, web pages, and so forth. For example, Google Desktop uses different indexers for different types of files. Once data is indexed, 
a user can search through his local files in the familiar Google interface. The current version of Google Desktop can index emails from Gmail, Outlook, Outlook Express, and Thunderbird; Microsoft Office, text, PDF and HTML documents; AOL and MSN Instant Messenger chats; and the web-pages users view in Internet Explorer and Mozilla Firefox. Google Desktop search is done using a web server installed on the local machine.

All of these tools help speed up searches by applying indexes on file. However, users are bounded to the index built up automatically by these tools, they do not have the freedom to classifying information according to their own taste. Moreover, due to the nature of full-text indexing applied by these tools, attributes with little semantic meanings are indexed along with those with real semantic meanings, therefore the index files become bigger files and occupy a large space, e.g., Google desktop requires 500 megabytes of disk space [30]. Additionally, there are some web security issues involved in Google Desktop Search. Moreover, all of these tools do not provide a mechanism to recognize relationships between individual file attributes.

\subsection{Category-based Applications}

\subsubsection{Categories in Microsoft Outlook}

Microsoft Outlook is a solution for managing and organizing e-mail messages, schedules, tasks, notes, contacts, and other information. On top of the traditional directory (folder) management, Outlook provides nice features that uses categories to keep track of different types of items that are related but stored in different folders. For example, users can keep track of all the meetings, contacts, and messages for a project, by creating a category named by that project and then further assign items to it. Categories also give users a way to keep track of items without putting them in separate folders. For example, users can keep business and personal tasks in the same task list and use the Business and Personal categories 
to view the tasks separately. Figure 3.2 also shows an example where contacts in Outlook can be assigned to different categories at the same time, such as "Hot Contact", "Personal", and "International", etc. Outlook supplies a list of categories, called "the Master Category List". Users can use this list as it is or add their own categories to it. The master list is stored as part of the Windows Registry. Users can import and export the master category lists between different computers.

\section{A contact exists in multiple categories}

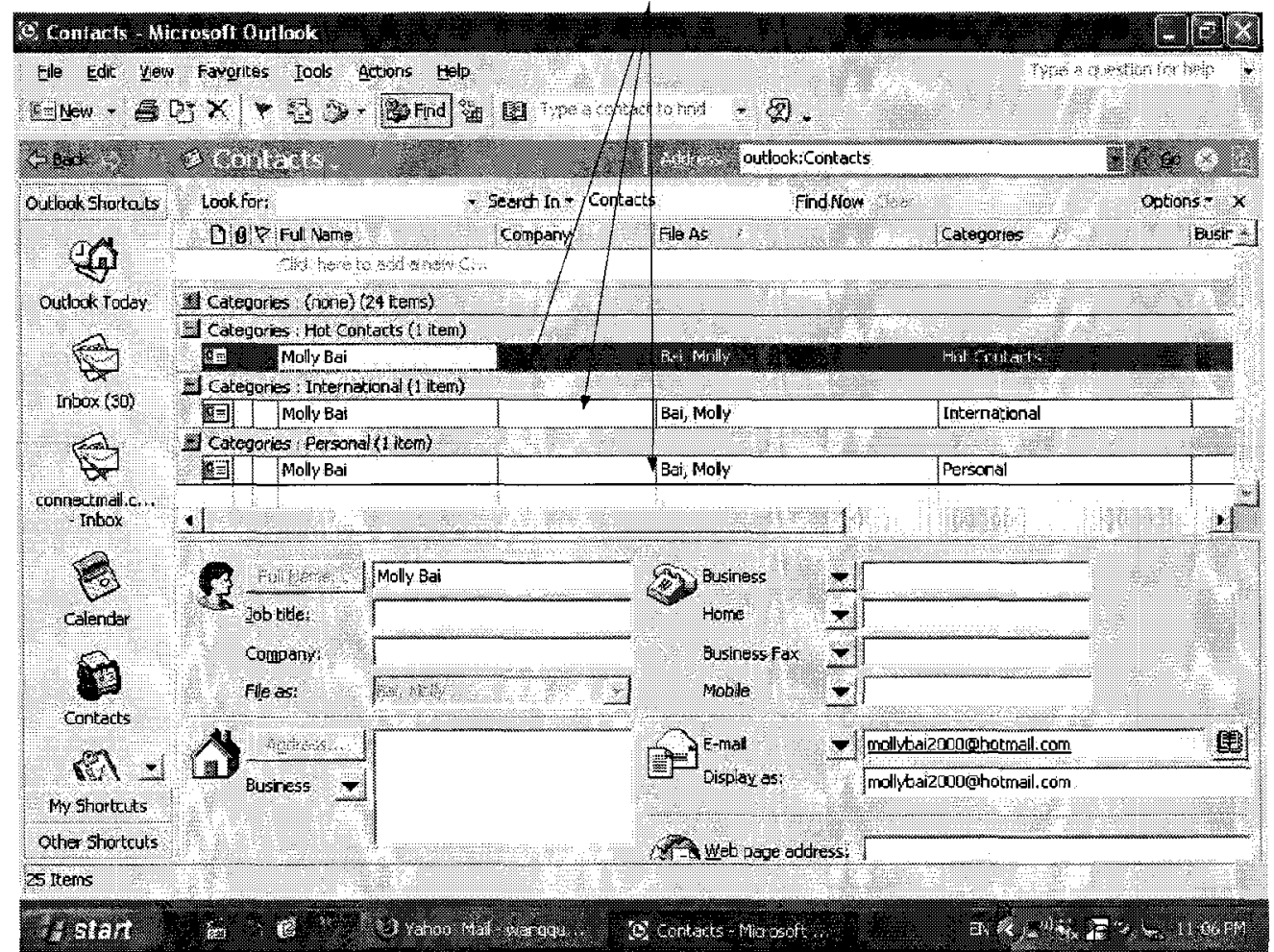

Figure 3.2: Multiple Categories in Microsoft Outlook

Microsoft Outlook provides the category management tool as a very nice feature to help organize its items in a more flexible and convenient way. However, it still has some drawbacks. The categorization structure forms a multiple categorization structure (i.e., one 
level flat categorization structure), which has two major disadvantages as we mentioned in the previous section. Meanwhile, the category management tool does not provide any means to recognize the relationship between each individual category.

\subsubsection{Bibliographix}

Bibliographix [39] is a software package developed for organizing references. It is very useful for assisting research projects. It facilitates both manually and automatically generating bibliographies, and organize them in a multiple categorization structure. In Bibliographix's References Management Module, reference records are created into a database for later retrieval. In its "Handling Projects" interface, users can generate different reference lists for each research project by adding (or deleting) bibliographic reference records to different research projects. As shown in Figure 3.3, there is only one "The Evolution of Cooperation" record in the database, however, it has been categorized to project "evolution" and "test" at the same time. The interface is also very straight forward for adding/removing project and adding/removing reference record.

Bibliographix provides nice categorization features to assist research projects. However, the drawbacks of the category management in Bibliographix are still the disadvantages gained from a multiple categorization structure (i.e., one level flat categorization structure), and the inability at recognizing the relationship between each individual category.

\subsubsection{Automatic Categorization Tools}

Document and record management are very important for large corporations and organizations. It is worthless if people cannot easily find information when they need it. The problem is that this often needs a large number of employees to first save important documents, and then classify them in a correct way. In reality, this is time-consuming and expensive. The need for categorization is evident. In order to handle a massive volume of 
The reference "The Evolution of Cooperation" has been categorized to project "evolution" and "test" at the same time

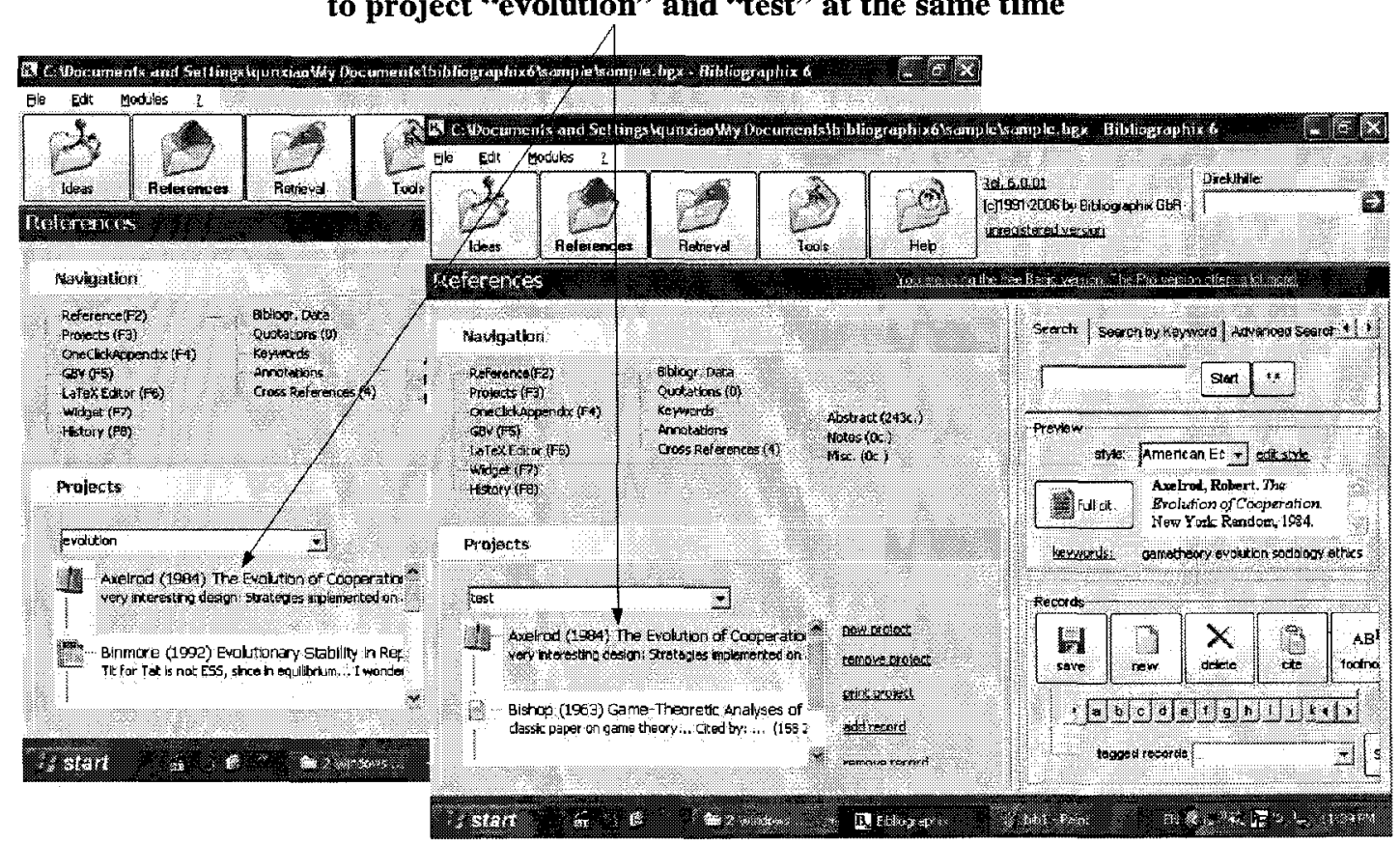

Figure 3.3: Multiple Categories in Bibliographix’s Handling Project Interface 
information in the industry, automatic categorization tools have come into place.

Automatic categorization is a software technology that assigns documents into related categories based on a variety of approaches [38]. These approaches includes:

- Rules-based categorization. Based upon some knowledge about the information, some rules can be built to assist in categorizing, and they are basically sets of IFTHEN rules set up by human editors, or subject matter experts. Examples of this approach are Verity's Intelligent Classifier [31] and Inktomi's Content Classification Engine module (CCE) [32].

- Catalog by example categorization. It uses training sets provided by humans to recognize if a document belongs to a certain category. The category is defined as patterns within a group of words. Examples are Inxight's Categorizer [33] and Mohomine's Moho Classifier [34].

- Statistical clustering categorization. It uses neural networks or co-occurrence of terms, finding clusters of files that are more closely related to each other and then assign those files to a category. Examples of this approach are Autonomy [35], Semio [36] and Mohomine [34].

Automatic categorization tools become popular because machines categorize faster than humans, and in evaluating the cost, auto-categorization seems to be cheaper than human categorizers. However, humans bring their knowledge of contexts into their task, they can do base decisions on context outside the information in the document, and also understand the context of the document. Although, humans are not as consistent as machines, they do a much better job than a machine in assigning documents to the right general category. Even if humans make mistakes, these mistakes tend to be understandable by other humans. Whereas, automatic categorizers can make mistakes that no one understands. Also if the quality of categorization results are poor, the cheap solution can be significantly more expensive eventually. Additionally, none of the vendors of these automatic categorization 
tools have claimed that they can be $100 \%$ automatic while doing the categorization work. In reality, these tools are significant aids in helping the human-assigned categorization tools to be more feasible. Based on their recommendations for categorization or metadata indexes assignment, users can make the final decision on using them or modifying them. However, most of these systems are fully developed and only suitable for large industrial corporations, who are able to afford the expensive fees for licensing and administrating. 


\section{Chapter 4}

\section{BASIC CONCEPTS}

\subsection{Category}

In the real world, we hear about the word "category" all of the time. But, what is a category? According to Wiktionary [40], a category is a group, often named or numbered, to which items are classified based on similarity or defined criteria. Within the domain of computer file systems, a category is a named group, to which files are assigned based on common characteristics or defined criteria. For example, we have saved several beverage product description files in the file system, such as "LiptonHerbalTea", "Pepsi", "PerrierMineralWater", "CoorsLightBeer", and "Merlot", etc. A name "Beverage" can describe the common property of these products, therefore, it can be used as a category name to classify them. A category illuminates a relationship between the subjects and objects of knowledge. A category name is a user defined name, which can be any noun or noun phrase. It should be meaningful to users and describes the commonality of objects that is classified to it. In the file system, a category is used to help users organize their files and serves as a guide during the search. We can also categorize files using some of the common properties of files as shown in the following examples: 
- The type of files, such as "pdf", "txt", "dvi", etc.

- The keywords in a document, such as: "graph theory", "file management", "unstructured data", etc.

- The URL of the source of a web document: "www.yahoo.com", "www.microsoft.com", "www.carleton.ca", etc.

- The subject of a document on what it is about, such as "Food", "Recipe", “TV Show", etc.

\subsection{Multiple Categorization Structure}

The multiple categorization structure is a flat categorization structure. In this structure, there is only one level of categories. These categories are independent from each other, and their knowledge domains are either disjointed or overlapping.

To give an example, as shown in Figure 4.1, "NonAlcoholicBeverage, "CarbonatedBeverage", and "AlcoholicBeverage" are three categories that are used to classify different beverage product. They form a one level flat categorization structure, and there is no relationship between any pair of them. Their knowledge domains are either disjointed or overlapping, e.g., "PerrierMineralWater" is classified to both category "NonAlcoholicBeverage" and "CarbonatedBeverage", since the knowledge domains of these two categories are overlapping each other. Meanwhile, no product is classified to "NonAlcoholicBeverage" and "AlcoholicBeverage" at the same time, as the knowledge domains for two categories are mutually exclusive from each other.

Although it might seem obvious that category "NonAlcoholicBeverage", "AlcoholicBeverage", and "CarbonatedBeverage" are related categories as their knowledge domains are all related to beverage, a flat categorization structure would not recognize their relationships and make the connection. As a result, the structure has two major drawbacks: 


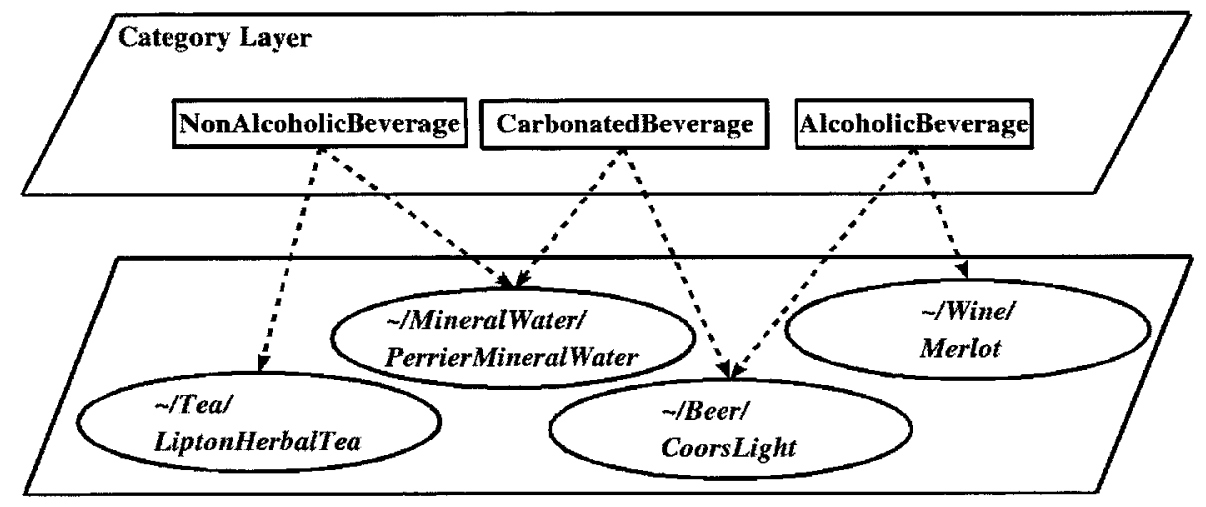

Figure 4.1: Example of Multiple Categorization Structure

1. If the categories tend to be fine-grained, the number of categories in the file systems may grow very large, and cause difficulties for searching possible categories, as a result, the category structure will go beyond what users can efficiently handle.

2. On the other hand, if the categories tend to be coarse-grained, the knowledge domain of each category may become very broad, as a result, the number of files classified to a certain category can grow very large as well. Users will still have difficulties to go through a sizable number of files and find their targets.

\subsection{Hierarchical Categorization Structure}

A hierarchical categorization structure is the widely adopted tree-structure for information organization. Comparing to a flat category structure, the hierarchical categorization structure recognizes the relationship between categories by allowing a category to be classified to another category, therefore, the knowledge domain from the upper level to the lower level becomes from general to refined, and the path information provided by this hierarchy acts as a guide helping users to find their targets.

The properties of the hierarchical categorization structure is as follows: 
- Any category in this structure can have zero or multiple sub-categories as needed.

- Every category other than the root category can only have at most one parent category.

- Objects can only be classified under one category

The directory hierarchy in the file systems is an example of this structure. In section 2.1.1.2, we have introduced its concept and examples.

Although the hierarchical categorization structure provides a very natural and helpful way for information organization, the nature of a tree structure has its own limitations. The following outlines some of the drawbacks:

1. By the nature of information organization, an object is more likely to have several different classifications, but the hierarchical categorization structure can only have one single classification for them.

2. The structure keeps the relationship between related objects by classifying them into a single category. However, if these objects are classified to different categories, their relationships then become lost. Later on, users may have difficulties finding them as they all scatter in different categories and become unorganized.

3. Using the hierarchical categorization structure, users are bounded to a static organization schema. While retrieving information, the static organization becomes a restriction. In particular, users must remember the ordered sequence of categories they used to organize the information when attempting to retrieve it by location, even though "the topics of interest during retrieval might be different from those during organization" [12].

4. If users forget the exact location that a target object is placed in the structure, they have to use brute force to search the whole category hierarchy, and check each category one by one to find it. 


\subsection{Multiple Hierarchical Categorization Structure}

In a multiple hierarchical categorization structure, the categories are also further grouped into various levels of categories. Furthermore, any category (except the root) can have one or multiple parent categories, which can be from the same or different levels. The multiple hierarchical categorization structure has a root category, which does not have any parent categories. Any category in this structure can have zero or multiple sub-categories as needed, which also can belong to different levels. Information objects can be classified to multiple categories with different levels as well, and users can access categories or objects through different paths in the hierarchy.

The featured properties of the multiple hierarchical categorization structure is as follows:

- A category or object can be classified to multiple categories.

- Every category (except root) may have multiple paths from the root.

- The same category may have different names, and the same object may have different names as well.

- Categories under a certain parent category may share the same name, and objects under a specific category may share the same name as well.

In order to demonstrate the concept clearly and completely, we use both Figure 4.2 and Figure 4.3. Figure $4.2^{1}$ is a general and easily understandable case for demonstrating the concept. Figure 4.3 is used to describe some special cases for the category names. In Figure 4.2, categories are grouped into five levels of categories. The lower the level is, the more granular or fine grained the categories are, and the higher the level is, the more coarse-grained the categories are. This forms a rooted categories hierarchy structure. As

\footnotetext{
${ }^{1}$ This example originates from [15]
} 
shown in Figure 4.2, from the root category " $/$ ", users can get the top level category, i.e., the category "Beverage". We can see that the category "Beverage" has ten objects classified to it, and it has been further grouped into "NonAlcoholic", "Alcoholic" and "Carbonated" as these categories fit three different aspects of the category beverage. Going further down into the hierarchy, the "Carbonated" category has six objects classified to it, and it has been further grouped into "Cola", "Beer", and "Champagne" as these categories are three different types of carbonated beverage. At last, we enter the "Champagne" category, and at there we find one object classified to it. By walking down into the lower level categories, the domains of knowledge are refined, irrelevant information are filtered, and users have been directed further closer to their target.

In addition, a category or object may have more than one name with regards to its different parent categories, and as a result, a category or object may have multiple categorization and multiple paths from the root category. We use Figure 4.3 to describe some special cases for the category names. In Figure 4.3, we have a target category which encloses the information of $3 \mathrm{D}$ game databases. We may classify this category to both "Databases" and "GraphicsSoftware". Under category "Databases", we name a category as "3DGame". In this way, we set up a category path "/ Database/ 3DGame" for our target category. Meanwhile, under "GraphicsSoftware", we name a category as "3D" to set the knowledge domain to 3D graphic software. Then, under "3D", we can name our target category with another name "GameDatabases", therefore, another path "/ GraphicsSoftware/ 3D/ GameDatabases" is set up for our target category. In this way, our target category has two names with regard to two different parents, and two corresponding path information as well. Moreover, categories may have the same name within our structure. For example, in Figure 4.3, we have the same category name "3D" under "Graphics" and "Animation", however, they represent different knowledge domains. "3D" under "Graphic" classifies all the information of 3D graphic software, whereas "3D" under "Animation" classifies all the information of $3 \mathrm{D}$ graphic animation software. The semantic meaning between them are 


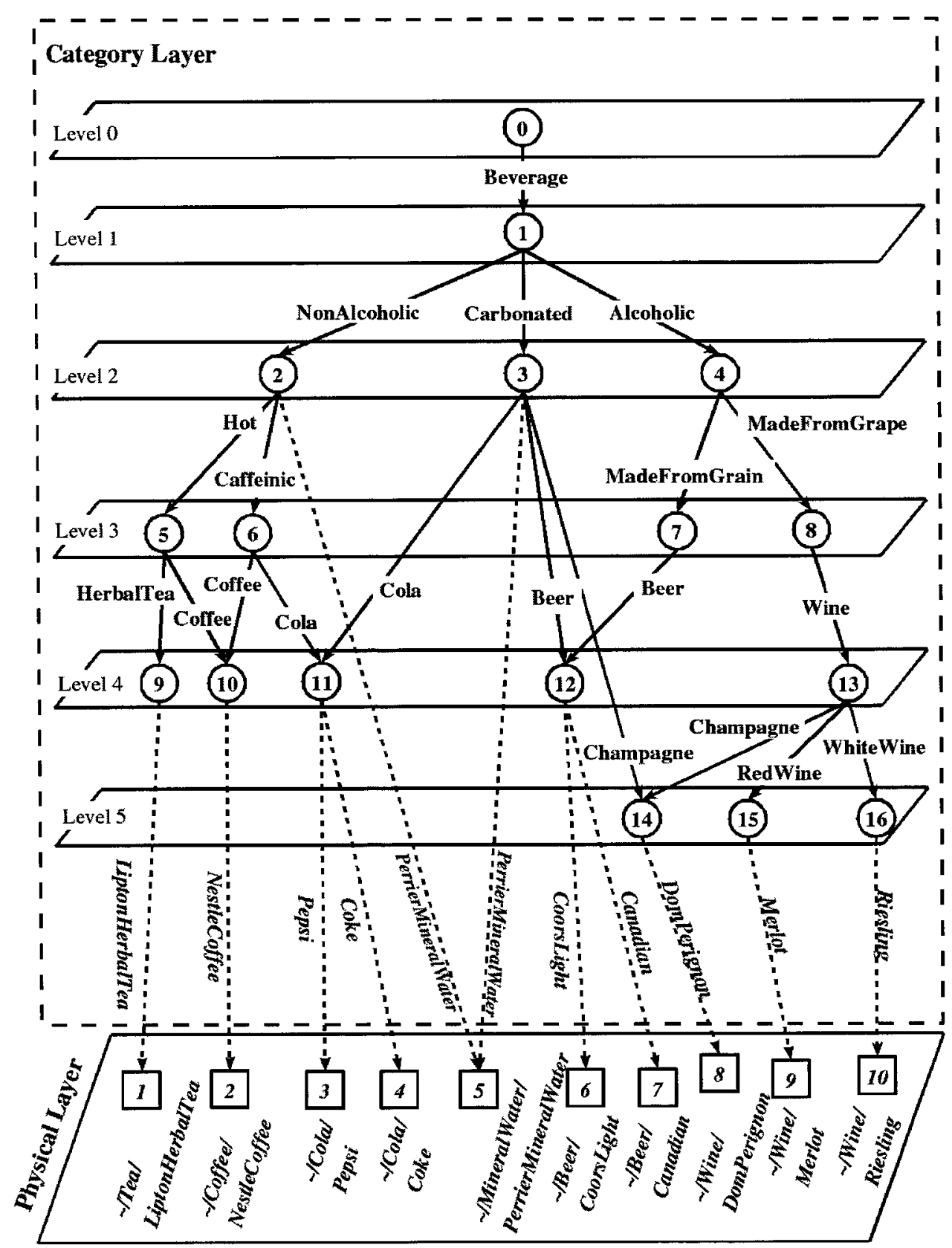

Figure 4.2: Example of Multiple Hierarchical Categorization Structure 
different because they are related to different parent categories and reside in different locations within our structure. Therefore, the name can not uniquely identify a category within our structure, it functions as the mapping of a category in its parent category.

In some hierarchical categorization structure, such as the directory hierarchy in file systems, the same name under a certain directory is not allowed. However, in our multiple hierarchical categorization structure, it is made possible. Different categories or objects under a certain category may share the same name. As shown in Figure 4.3, under the category "Programming", we named two different categories with the same name "Java". However, their knowledge domains are different, one refers to "MySQL java database programming", while the other refers to "Berkeley DB java database programming".

The relationship between categories can be through inheritance or composition. For example, we have category "Wine" being further grouped as "Champagne", "RedWine", and "WhiteWine", which are all wines (inheritance). We also can have "Company" being further grouped as "Sales", "Administration", and "Operations", etc., which compose a company (composition). Moreover, in our structure, there also exists another type of relationship which is neither inheritance nor composition.For example, in Figure 4.2, “/ Beverage/ Carbonated/ Cola" is related to non-alcoholic and caffeinated beverage, therefore we should create an alias (or a link) between these two knowledge domains, so that whenever a user retrieves information under "/ Beverage/ Carbonated/ Cola", they should also be able to get the alias information, i.e., "/Beverage/ NonAlcoholic/Caffeinated/Cola", and gather further information about the beverage. 


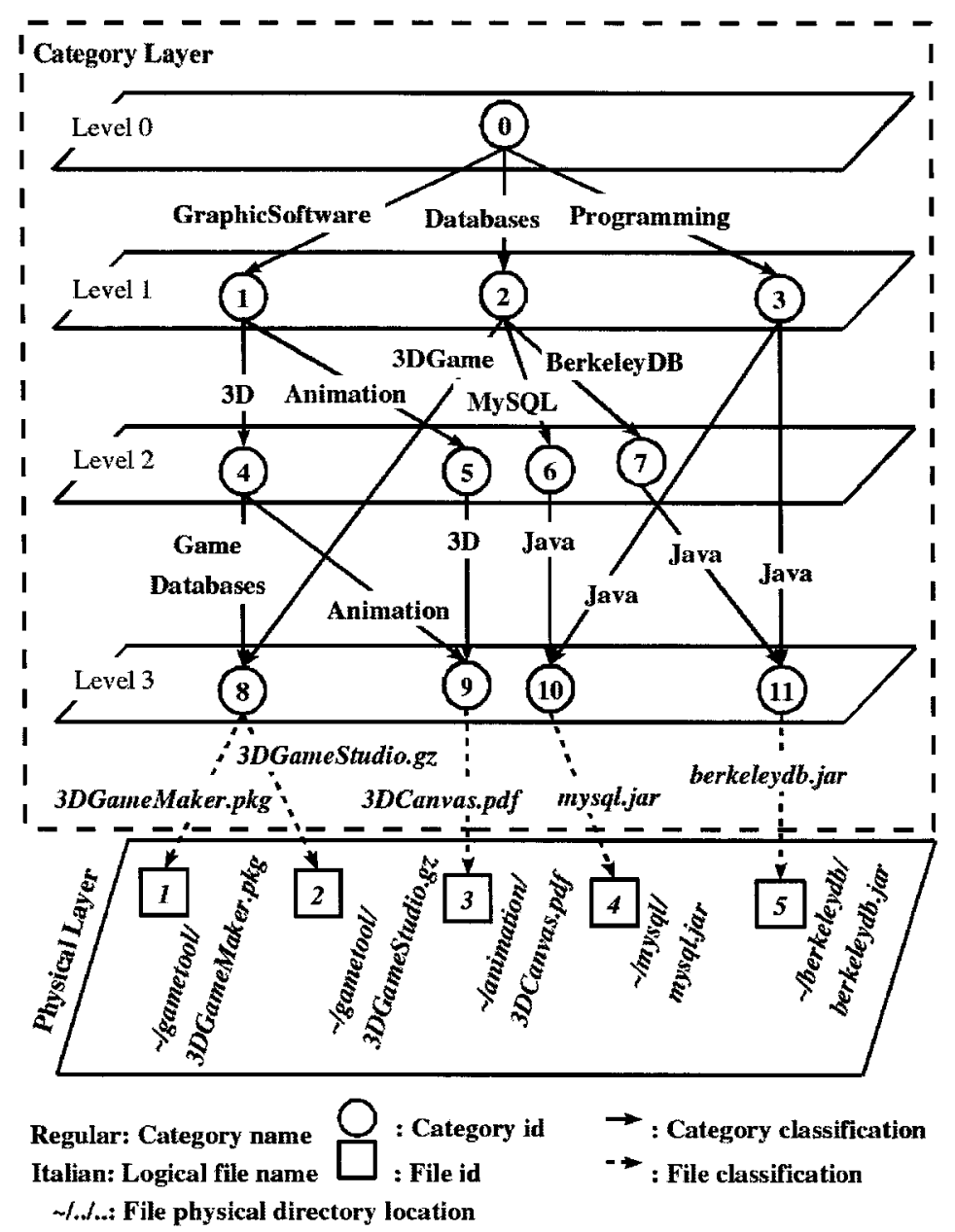

Figure 4.3: Example of Category Names 


\subsection{Formalization of Multiple Hierarchical Categorization Structure of File Systems}

As stated in objective (2) (on page 9) of this thesis, some of the basic concepts in CASH were defined inaccurately, e.g., category and category structure. Some concepts have not been defined, e.g., category name and logical file name. In the following, we improve the previous work by formalizing the multiple hierarchical categorization structure of file systems.

Category In the multiple hierarchical categorization structure, a category is an object which can have a group or set of files or categories. It can be illustrated as a category node in a directed graph. As shown in Figure 4.2, node $\mathbf{0}$ and $\mathbf{1 6}$ are all categories. The category is the fundamental structure concept in the multiple hierarchical categorization structure, and has several properties, i.e., unique category id, category mode (the access and execution permissions), creation time, time of last access, time of last modification, individual owner id and group id that is associated with it.

Category Name and Parent/Child Category A category name is a category classification of the category in the parent category. It is only meaningful and relevant with regard to its parent category. A category name is represented as a named directed edge in the directed graph. The head node of this edge represents the parent category, and the end node represent the child category. In Figure 4.2, "NonAlcoholic" is the name of the category $\mathbf{2}$ with regard to the category $\mathbf{1}$, therefore, the category $\mathbf{1}$ is the parent category, and the category $\mathbf{2}$ is the child category.

Logical File Name A logical file name is a file classification of the physical file in the specified category. It is only meaningful and relevant with regard to its specified category. 
We use the word "logical" to differentiate it from the real file name in the physical file system. In our structure, the logical file name can be any name that users choose to classify the physical file. The logical file name is represented as a named directed edge in the directed graph. The head node of this edge represents the specified category. The end node represents the file. In Figure 4.2, "Riesling" is the name of the file $\mathbf{1 0}$ with regard to its specified category 16.

Multiple Hierarchical Categorization Structure A multiple hierarchical categorization structure is a rooted directed-acyclic-graph (DAG)

$$
\mathbf{G}=(\mathbf{C} \cup \mathbf{F}, \mathbf{N}, \mathbf{r}, \mathbf{l}, \mathbf{p}, \text { in, out })
$$

where $\mathbf{C}$ is a set of categories, which are identified by category id and $\mathbf{F}$ is a set of files, which are identified by file id,

$\mathbf{N}$ is a set of named edges for categories and files, $\mathbf{r} \in \mathbf{C}$ is the unique root of the graph,

$\mathbf{l}$ is a function that maps a category to a number which is the level of the category in the graph,

$\mathbf{p}$ is a path function that maps the category to its paths from the root, in is a function that gives the in-degree of a node, out is a function that gives the out-degree of a node, such that the following hold

(1) $\mathbf{l}(\mathbf{r})=0$; i.e., the highest level has the least level value.

(2) For each $c \in \mathbf{C}$ other than $\mathbf{r}, \operatorname{in}(c) \geq 1$ and out $(c) \geq 0$; i.e., a category other than root must have a parent category and can have 0 or more child categories and files.

(3) For each $f \in \mathbf{F}, \operatorname{in}(f) \geq 1$ and $\operatorname{out}(f)=0$; i.e., a file must have a specified category.

(4) Let $c, c_{1}, c_{2}, \ldots, c_{n} \in \mathbf{C}$ such that for each pair $c_{i}$ and $c$, there is a directed edge $n_{i} \in \mathbf{N}$ from $c_{i}$ to $c$; i.e., $c$ is a child category of $c_{i}$, and then $\mathbf{l}(c)>\max \left(\mathbf{l}\left(c_{1}\right), \mathbf{l}\left(c_{2}\right), \ldots, \mathbf{l}\left(c_{n}\right)\right)$ and $\mathbf{p}\left(c_{i}\right) \subseteq \mathbf{p}(c)$ for $1 \leq i \leq n$. 
We also use Figure 4.2, to show an example. Category $\mathbf{0}$ is the root of the structure and at level $\mathbf{0}$. The circle nodes represent categories, and the rectangle nodes represent files. A category has at least one parent category and zero or more child categories and files. A file has at least one specified category. Category $\mathbf{1 4}$ is at level 5, which is lower than the level of its parent category 3 (at level 2) and $\mathbf{1 3}$ (at level 4). The paths of category 11 includes both ts parent categories” paths (“/ Beverage/ NonAlcoholic/ Caffeinated/ Cola" includes "/ Beverage/ NonAlcoholic/ Caffeinated/", and "/ Beverage/ Carbonated/ Cola" includes "/ Beverage/ Carbonated").

\subsection{Featured Properties}

The featured properties of the Multiple Hierarchical Categorization Structure are as follows:

Multiple Categorization Both files and categories can be classified to one or more categories. As shown in Figure 4.2, both file and category are classified to one or more categories. For example, the category 10,11, 12, 14 and the file 5 are all classified to multiple categories.

Multiple Paths Every category other than the root category may have multiple paths from the root category $\mathbf{1}(/)$. In Figure 4.2, the category $\mathbf{1 1}$ has two paths from the root, " Beverage/ NonAlcoholic/ Caffeinated/ Cola", and "/ Beverage/ Carbonated/ Cola". Multiple paths is a novel feature that provides users with more choices to locate their target in a fast and convenient way. It also makes it easier for users to find related categories and files of interest within them. For example, by examining the above two paths of the category 11, the users' intention of finding information related to "Cola" results in additional path choices or options that includes "NonAlcoholic" and "Carbonated". This thereby broadens 
the information on the search "Cola" to include additional information and choices.

can soon be swift to information related to "NonAlcoholic" or "Carbonated" as they provide users with more choices.

Same Category/File with Different Names The same category may have different names with regards to different parent categories. As shown in Figure 4.3, the category 9 has the name "Animation" with regard to its parent category 4, and another name "3D" with regard to its parent category $\mathbf{5}$. This property is a nice feature for naturally organizing and searching files. Users have freedom to classify a target category with different names according to their wishes, as a result, the target category may have multiple paths. This makes it easier to find the category and search its knowledge domain.

Different Categories/Files with Same Name As opposed to a hierarchical categorization structure, which does not allow for the same name to be under a certain category, our multiple hierarchical categorization structure makes no restriction on this matter. Different categories or files under a certain category may share the same name. As shown in Figure 4.3, category 10 and 11 share the same name "Java". However, the knowledge domain of these two categories are different: the category 10 refers to "MySQL java database programing", and the category 11 refers to "Berkeley DB java database programming". Another nice characteristic of this feature is that, these categories with the same name may have different priorities, so if an application needs to utilize the information under these categories, it can go into one of these categories based on its priority level, and search for information under it. 


\section{Chapter 5}

\section{DESIGN OF THE CASH2 SYSTEM}

In this chapter, we describe the existing problems of the previous CASH design and implementation work, and our improved system design to solve these issues with better solution.

\subsection{CASH Design and Issues}

\subsubsection{Database Schema}

MySQL database engine has been chosen as the back end storage engine in CASH. The database schema is shown in Figure 5.1.

The Category-Hierarchy table is used to show the relationship between categories. It is composed of four fields: "CID" is category id, "Parents" and "Children" are lists of $<$ category id $\mid$ category name $>$ pairs, e.g., $<2|a><4| b><5|c\rangle$, "Desc" is the description of the category.

The Category table is used to store category information. It is composed of two fields: "CID" as category id, and "CName" as category name. Different CID can have the same CName.

The Directory table saves all useful physical information in the file systems about 
directories. "Dino" is the inode number, "DPath" is the absolute directory path, "Mode" is the access mode, "Mtime" is the time of last modification, "Size" is the size of the directory, "Links" is the number of links the directory has, "Uid" and "Gid" is the user id and group id of the directory. The directory table is created to help locate a physical file in the file system by providing the absolute directory path of that file.

The Files table saves file information and their categorization information. "FName" is the name of a file without path, "DID" is the directory id of the file, which is actually the inode number of the directory, "Mode" is the access mode, "Ctime" is the creation time, "Atime" is the time of last access, "Mtime" is the time of last modification, "Size" is the file size, "Links" is the number of links the file has, "Uid" and "Gid" is the user id and the group id of the file, "Inode" is its inode number, and "Desc" is a short description of the file. The last field is "Categories", which is a string that composed of a list of <category id $\mid$ category name $>$ in a path order. An example is $<6|\mathrm{C}++><7| \mathrm{JAVA}><8 \mid \mathrm{SQL}>$, which means the file is classified under category path / C++/ JAVA/ SQL.

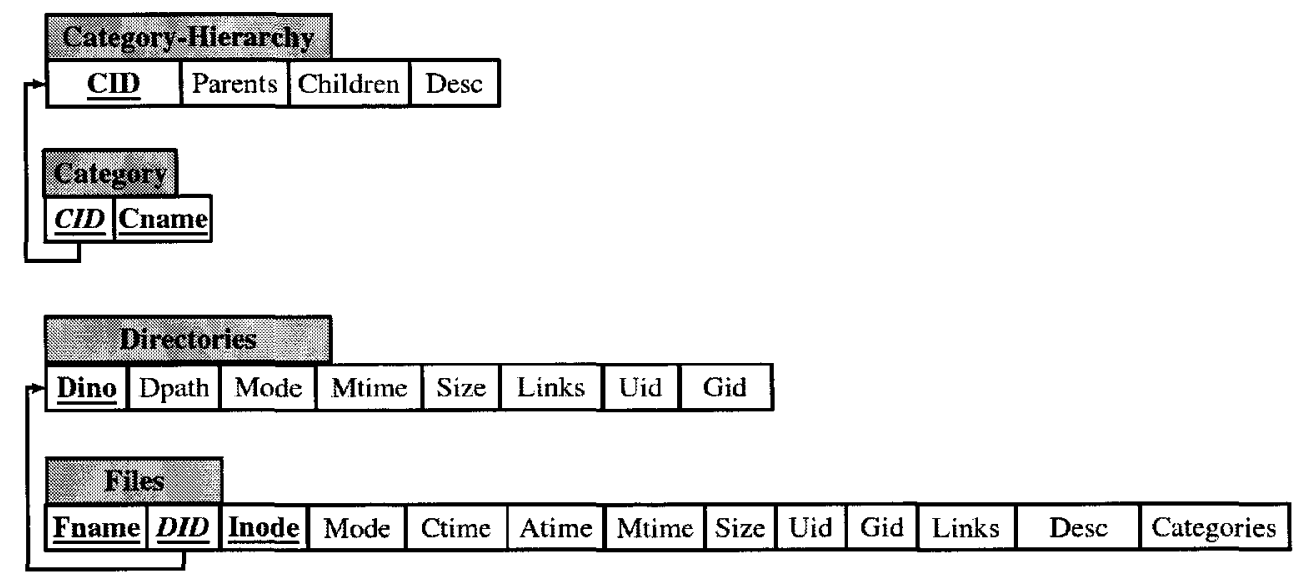

(bold underline $=$ primary key, italic $=$ foreign key)

Figure 5.1: Database Schema In CASH 


\subsubsection{Major Issues}

The design in CASH does not follow the basic theory of Entity-Relationship modeling method. Therefore, it leads to some major issues for the later implementation.

\section{Hierarchical Multiple Categorization Structure Modeling}

We mentioned in section 4.5 that from a DAG point of view, a category name is a name of a directed edge that connects two category nodes. Therefore, it is only meaningful and relevant when both parent category and child category is present. However in Category table, there are only two fields, "CID" and "Cname"; the parent category information is lost, so that the relationship between category was interpreted incorrectly. For example, we are trying to represent two category paths in the hierarchical multiple categorization structure, / a / c, and / b/d, as shown in Figure 5.3(a). The sample Category and Category-Hierarchy information stored in the database is shown in Figure 5.2. Because of the errors in the Category table schema, the interpreted relationship between these categories can only be drawn as in Figure 5.3(b). When a user issues a command to read the paths information from category $\mathbf{1}$ to $\mathbf{3}$, they actually get four paths, / a/ c, / a/ d, / b/c, and / b/ d, which is incorrect.

Cateory-Hierarcuy
\begin{tabular}{|c|c|c|c|}
\hline CID & Parents & Children & Desc \\
\hline 1 & Null & $\langle 2 \mid \mathrm{a}\rangle\langle 4 \mid \mathrm{b}\rangle$ & Null \\
\hline 2 & $<1 \mid />$ & $<3|\mathrm{c}\rangle$ & Null \\
\hline 3 & $<2|\mathrm{a}\rangle\langle 4 \mid \mathrm{b}\rangle$ & Null & Null \\
\hline 4 & $<1 \mid />$ & $<3|\mathrm{~d}\rangle$ & Null \\
\hline
\end{tabular}

Category
\begin{tabular}{|c|c|}
\hline CID & Cname \\
\hline 1 & $/$ \\
\hline 2 & $\mathrm{a}$ \\
\hline 3 & $\mathrm{c}$ \\
\hline 3 & $\mathrm{~d}$ \\
\hline 4 & $\mathrm{~b}$ \\
\hline
\end{tabular}

Figure 5.2: Sample Category table and Category-Hierarchy table

\section{Sequential Search In Database}

As we explained in Section 5.1.1, the field "Categories" in File table is a character 


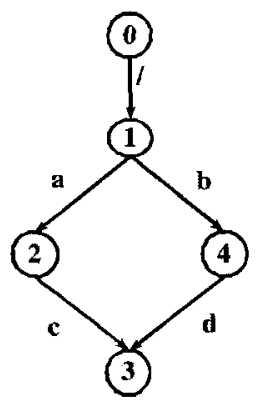

(a)

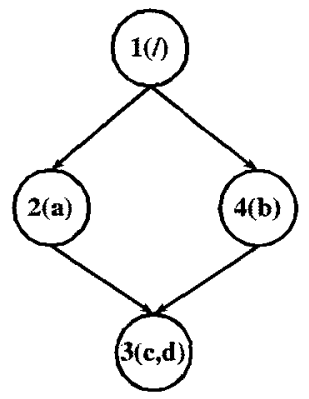

(b)

Figure 5.3: Graph Representations of Categories and Category-Hierarchy In CASH

string that stores a list of $<$ category id $\mid$ category name $>$ in a path order. Every time users issue commands to get all of the file information under a certain category name, the implementation program has to go into the table File, and sequentially search the field "Categories" for each record to find a character string that matches with the given category name. The sequential search is no different than traversing the whole directory hierarchy. The same problem occurs when users try to rename a category name, update a file information under a category name, delete a category, delete a category name or copy the classification information of a category. Further more, the Category_hierarchy table has two fields: "Parents" and "Children", whose value are string characters that store a collection of $<$ category id $\mid$ category name $>$ pairs. Apparently, the information of categories is repeatedly stored in both fields. Although the advantage of keeping both "Parents" and "Children" fields is that once a category is located, all the information about its parent categories and child categories is retrieved from database at once. But it causes a lot of database information redundancy and uses disk space as a trade off. This eventually slows down the performance of category information retrieval.

\section{Database Primary Key}


In the Directory table, "Dino" is defined as the primary key, but a directory in the file systems is uniquely identified by two values. One is the directory inode number, which is captured as "Dino" in the existing schema. and the other value, i.e., the device number, which identifies the file system that a directory locates at, has been missed out in the existing schema. As a result, the primary key in the Directory table is defined incorrectly. Consequently, the schema of the table File is defined wrong as well, since the "Dino" in Directory table is the foreign key in the File table.

\section{Engine Selection}

MySQL has been chosen as the back end database engine to store all the categories and its related information in CASH. MySQL is a popular open source relational database tool that applies a client server architecture, centralizes the database management in server side, and enables users to remotely access the database service via client interface and an application. However, CASH is developed for UNIX/Linux personal computer users. The application is running on the same machine that users reside, therefore, a client-server database is not necessary. In addition, users do not interact with back end database engine directly. The implementation program takes care of all the queries, and as a result, there is no dynamic queries issued from the users. Hence, a SQL query layer is not needed either. MySQL is obviously too heavily weighted for CASH applications. In section 5.2.3, we will detail our study on the features of different database engines, and explain our new choices.

\subsection{Our Approach}

As stated above and in objectives (4) and (5) of this thesis (on page 9), CASH has proposed a database model and realized its multiple hierarchical categorization structure. However it does not explain what methodology it applies for modeling the database while struggling 
with a structure that was poorly designed. Furthermore, it does not study different database engines nor choose the one that best fits the application of CASH. In this section we explain our modeling methodology, our choice of the storage engine, and our improved design in CASH2.

\subsubsection{System Architecture}

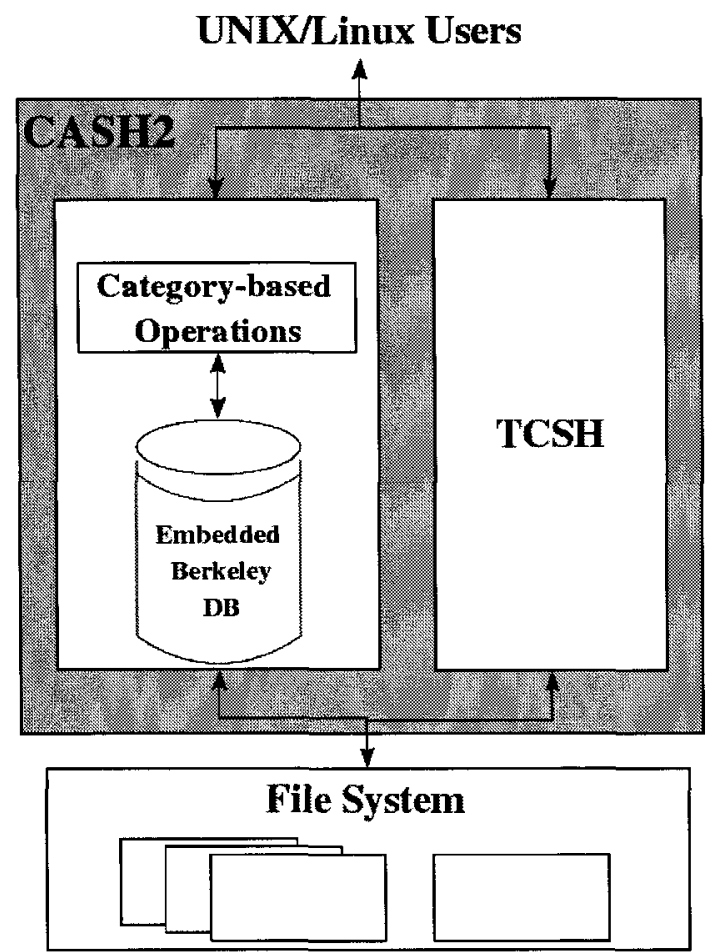

Figure 5.4: System Architecture

The system architecture of CASH2 is shown in Figure 5.4. CASH2 is written within the existing code package of TCSH version 6.12.00. It enables a multiple categorization management tool by building up a set of utility commands to implement category-based operations at the front end, and a category and file database at the back end of the system. $\mathrm{CASH} 2$ transparently transform those commands into queries, and returns the query results 
from the database back to users. CASH2 inherited all the functionalities of TCSH, so from this point of view, users should not notice any difference between CASH2 and TCSH. Our multiple categorization management tool acting as an add-on utility brings more options to users when they organize and retrieve their personal files.

\subsubsection{Modeling Methodologies}

As a multiple categorization file management tool, the design goal of $\mathrm{CASH} 2$ is to realize the multiple-categorization hierarchy structure in its application. To model this structure, we have studied two widely used modeling methodologies: Entity-Relationship Modeling and Object-Oriented Modeling. In Entity-Relationship Model (ER Model) [16], entities are information objects in the problem domain. Entities, their attributes and relationships between entities are represented in the form of ER diagrams. ER modeling allows us to store conceptual-level descriptions of the data in terms of entity and their relationship, into a database with a logical schema [14]. As a data model for high-level descriptions of conceptual data models, ER modeling has been widely used to develop a database design. Object-Oriented Modeling [17, 18, 19, 20] applies the concept of software classes to identify and model the objects in the problem domain, as well as the attributes of each class and the relationships among classes. The major difference between classes in static object oriented modeling and entities/relationships in ER modeling is that classes have operations but entities and their relationships do not. In addition, object oriented modeling also applies advanced information modeling concepts of aggregation and generalization/ specialization.

In CASH2 we choose ER modeling as our modeling methodologies for the following reasons:

1. ER modeling lacks the ability to model behavior of each entity, but it is adequate for modeling CASH2. The primary design goal of $\mathrm{CASH} 2$ is to realize the hierarchical 
multiple categorization data structure, and data modeling. This is what ER modeling is specialized for.

2. $\mathrm{CASH} 2$ uses the $\mathrm{C}$ programming language, which is a native language that is well known for its efficiency. $\mathrm{C}$ is the most popular programming language for writing system software. However it lacks some of the features that object-oriented programming languages have, such as class inheritance, and so forth. As such it is not suitable to use $\mathrm{C}$ language to implement an object oriented model.

\subsubsection{A Study Of Database Engines}

\subsubsection{Candidate Databases}

By applying ER modeling for the system design, $\mathrm{CASH} 2$ has to choose a database engine at the back-end to store all the information in categories, files and directories, and their relationships. Which engine would best suited for the implementation and functionality of $\mathrm{CASH} 2$ ? In the following sections we answer this question, and study different database engines while explaining our choice.

To choose a database engine for $\mathrm{CASH} 2$, we are mainly looking at the following criteria:

\section{Non-embedded vs Embedded}

There are two kinds of system architecture for a database, Non-embedded and Embedded. Almost all commercial database products are implemented as non-embedded database systems. They build a server to which remote clients connect, and the server does all the data management within it. An embedded database system builds an embedded database library and a simple function-call API. The library is linked directly into the application, and does all the database management locally [41]. Nonembedded databases have become very popular because the servers are centralized 
and managed by a separate process. This leads to a simple implementation, clean separation of functionality, and a more flexible and a more friendly user-interface running on inexpensive client machines rather than on the server [14]. However, centralized administration also means whenever an application is shipped and installed, the end user needs to install and administer a separate database server. The programmer must support not just one product, but two. In addition, adding a server process to the application can cause chances for installation problems and run-time errors [41]. In CASH2, the implementation program does all the work to query the database. We do not need a server administrator or a sophisticated client interface. A separate database server process running constantly and waiting for queries is unnecessary. For this reason, an embedded database that links into the application, runs locally and works as a component of the system is the best choice for CASH2.

\section{SQL Query Layer Not Needed}

In CASH2, we develop UNIX like commands for users to query the category databases. This transparently transfer a user command into queries and returns the result back to users. Since there are no dynamic queries involved, there is no need for a database that has an SQL query layer to perform complicated searches. A database that provides sophisticated storage facilities and provides fast and easy APIs for querying is adequate for the applications of CASH2.

\section{Fixed-length vs Variable-length}

When storing a record into the database, variable-length record is preferred because a fixed-length record occupies a fixed length of space on the hard disk. This decreases the utilization of hard disk space and cause the overhead of pre-setting the size of array.

\section{Fast, Easy, and Small}


We prefer a database that is written is $C$ language, easy to download and install, runs fast with a small footprint, and has a minimal external configuration.

\section{Concurrency Control}

In CASH2, the root user has the authority to classify all the files in the file system. Normal users are allowed to either build their own category database or utilize the one that the root user created to manage their own categories within it. In the latter case, we need a database which is able to provide sophisticated concurrency control that allows multiple users to access it at the same time.

\section{Free}

A database that is free for non-commercial use and open source is preferred.

Based on the above requirements we have included the following open source embedded databases in our brief survey.

\section{Embedded Firebird SQL}

Based on the beta version of InterBasev6.0 database that was published by Borland Software Corporation, a group of developers adopted the code and begun an independent development of Firebird [47]. Firebird is a relational database offering many ANSI SQL-99 features that runs on Linux, Windows, and a variety of Unix platforms. The major drawback of the embedded version of Firebird is that it does not allows multiple programs to concurrently connect to the same database. This is not the case for Firebird in client/server mode, but, in embedded mode, only one program can connect to the database at a time. In addition, it's SQL layer is unnecessary to the applications of CASH2.

\section{SQLite}


SQLite [49] is a C library that implements an embeddable SQL database engine. The library implements a large subset of SQL-92 standard. In SQLite, both the database engine and its interface are within a single library. The database also has the ability to store all the data in a single disk file. This solution claims to be simple to administer, operate, maintain and embed into large programs. We did not choose SQLite for the following two reason: first, the currency control has a drawback that it currently has only database-level locking, which is coarse grained. Second, it has a SQL layer which CASH2 does not need it.

\section{Derby}

IBM has developed a Java-based SQL database engine "CloudScape" as an open source project named "Apache Derby Project" [50]. It is a relational database implemented entirely in Java. Derby provides an embedded JDBC driver that allows users to embed Derby in any Java-based solution. It has a small foot print for the base engine, an embedded JDBC driver, and is easy to install, deploy, and use. However, Derby only allows a single process to have the database open at a time, so multiple users cannot access the database at the same time. Moreover, it development language, Java, does not not meet the requirements of CASH2 as well.

\section{Embedded MySQL}

MySQL [51] is a popular relational database, and the implementation of CASH is based on the client-server version of it. As we mentioned above, we needed to choose an embedded database for $\mathrm{CASH} 2$, as such we are looking at the embedded version of MySQL. Embedded MySQL [51]server library runs a full-featured MySQL server inside a client application. It supports a broad subset of the ANSI SQL 99 syntax, and advanced permissions and security systems,etc, thus the embedded library has a pretty large footprint (e.g., embedded MySQL server library builds on MyISAM engine is of the size of 44 megabytes), and many features are not necessary, and 
hence this becomes overhead on $\mathrm{CASH} 2$, such as SQL and advanced permissions supports.

\section{5. db.*}

The db.* [48] database management system is designed to be embedded for developing $\mathrm{C}$ language database applications. It combines the network and relational model technologies, and does not have a SQL query layer. The emphasis of $\mathrm{db}^{*}$ is on small size. $\mathrm{db}^{*}$ could have been the choice of database for CASH2, however, a shortcoming of $\mathrm{db}^{*}$ is that it uses a fixed-length record size for all records in a singe table, which does not meet the requirements of CASH2.

\section{Berkeley DB}

Berkeley DB [41] is an open source embedded database library. It is mainly a data storage layer, and does not support SQL and schema. The size of the Berkeley DB library built with current GNU gcc compilers is in the range of $425 \mathrm{~KB}$ to $700 \mathrm{~KB}$ on 32-bit x86 architectures, and in the range of $520 \mathrm{~KB}$ to $820 \mathrm{~KB}$ on 64 -bit $\mathrm{x} 86$ architectures, hence it has a small memory footprint, and is much faster. Berkeley DB library also provides scalable concurrent data management services to applications and a simple function-call API for data access and management.

Due to storage shortage, concurrency control drawback, or unnecessary SQL layer overhead, Embedded Firebird SQL, SQLite, Derby, Embedded MySQL, and db.* do not fulfill the requirements for $\mathrm{CASH} 2$. As a result, we narrowed down our choice to Berkeley DB, and a brief introduction of it is covered in the next section.

\subsubsection{A Brief Introduction Of Berkeley DB}

Berkeley DB(BDB) [41, 42] by Sleepycat Inc. is an open source embedded database library written entirely in ANSI C. It links directly into the application, and runs in the same 
address space as the application. There is no inter-process communication between processes on the same machine when they perform database operations. BDB also does not support the SQL query. This eliminates the overhead of query parsing, optimization, and execution. On the other hand, it results in more requirements for the programmer. They must understand the data structures and their representation, and they must write the code to do the work. By doing so, the application can run very fast. In addition, Berkeley DB does not have the notion of schema and data types as those in relational systems. It uses (key, value) pairs to identify records in the database and programmers need to present a key to identify the (key, value) pair when they call a Berkeley DB interface and issue database operations, such as insert, delete, find and update a record. The (key, value) pairs can have very rich internal structure, as much as ANSI C can provide, but the library is not aware of that, and only the programmers and applications know the structure and can operate on it. Moreover, If more than one application links in Berkeley DB, then more than one user can use the same database at the same time, the library handles coordination among the applications, and guarantees that they do not interfere with one another.

As shown in Figure 5.5, BDB is divided into five subsystems.

\section{Access Methods}

The access methods subsystem offers four access methods for creating and accessing database files: B-tree [44], Hashing [45], Queue and Recno. Recno is built on B-tree method and it is designed to provide better support for processing document data, e.g., data in text files [46]. All access methods store (key, value) pairs. In B-tree and Hash methods, the stored records can be of any type that the programming language support. The records are found based on the keys and comparisons which are made on the keys e.g. to solve the order of the records. In queue and Recno methods, the keys are determined by the system. Furthermore, the values in the queue method are limited to a fixed length. There is little difference in performance between the Hash 


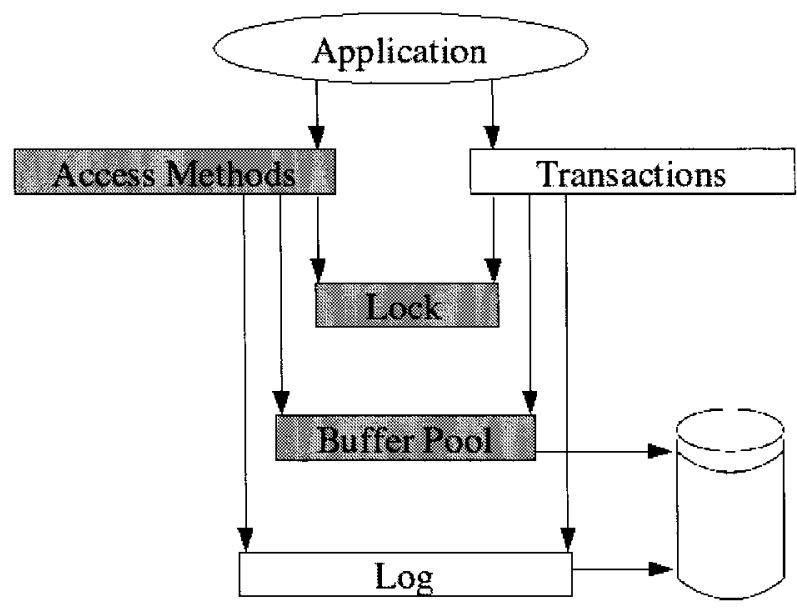

: Berkeley DB Subsystems used in CASH2

Figure 5.5: Berkeley DB Subsystems

and B-tree access methods on small data sets. For data sets larger than the cache, it is recommend to use the Btree access method, since the sorted order of records in B-tree helps access keys near to the key being accessed [41]. In CASH2, we choose B-tree access methods for BDB.

\section{Memory Pool}

The Memory Pool subsystem provides a general-purpose shared memory buffer pool used by any number of processes or threads within the processes to share access to the databases [41]. The database page size is configurable and determined at the database creation time. Locking happens on page level, and changing the page size affects the number of locks acquired when a record is accessed. In CASH2, we enable the memory pool subsystem in BDB to allow shared file access between different users in a same category database.

\section{Transaction}


The Transaction subsystem offers tools to implement a transactional recoverable database system. It allows a group of database changes to be treated as an atomic unit so that either all of the changes are done, or none of the changes are done [41]. In $\mathrm{CASH} 2$, we do not enable this in BDB because it is a heavy-weighted subsystem, and not all the file systems need to be transactional recoverable.

\section{Locking}

The Locking subsystem is the general-purpose lock manager. In BDB, Locking happens on page level. The database page size is configurable and determined at the database creation time. Locking happens on page level, and changing the page size affects the number of locks acquired when a record is accessed. However, if the page size is set smaller than what is the file system block size, the performance might suffer. By default, BDB sets the page size to be the same as the file system block size. In $\mathrm{CASH}$, we enable the locking subsystems in $\mathrm{BDB}$ to allow shared database file access between different users.

\section{Logging}

The Logging subsystem is the write-ahead logging used to support the Berkeley DB transaction model. We do not enable the transaction subsystem, as such we do not enable it in BDB.

\subsubsection{Database Design}

The very first step of database design is the Requirements Analysis. We need to understand what data is to be stored in the database, and what application must be built on top of it. The second step is the Conceptual Database Design. All the information gathered in the requirements analysis step is used to develop a high-level description of the data to be stored in the database. The last step is the Logical Database Design, where we choose a 
DBMS to convert the conceptual database design into a database schema. [14].

We have completed our first step in design in chapter 4. In the following two sections, we describe our work to accomplish the second and the third steps.

\subsubsection{Conceptual Database Design}

In the stage of conceptual database design, we represent the hierarchical multiple categorization structure in CASH2 with the ER model diagram. As shown in Figure 5.6, there are three entities and three relationships in the model.

\section{Entities:}

\section{- Category}

It represents the category node in the the rooted directed-acyclic-graph, and consists of seven attributes:

Cid: a numerical id by which each category is uniquely identified.

Cmode: Access and execution permission associated with the category.

Cctime: Creation time of the category.

Catime: Time of the last category access.

Cmtime: Time of the last category modification.

Cuid: Owner id for the category.

Cgid: Group id for the category.

\section{- File}

It represents the file node in the the rooted directed-acyclic-graph, and consists of ten attributes:

Fdev: Device number of the file.

Fino: Inode number of the file. 


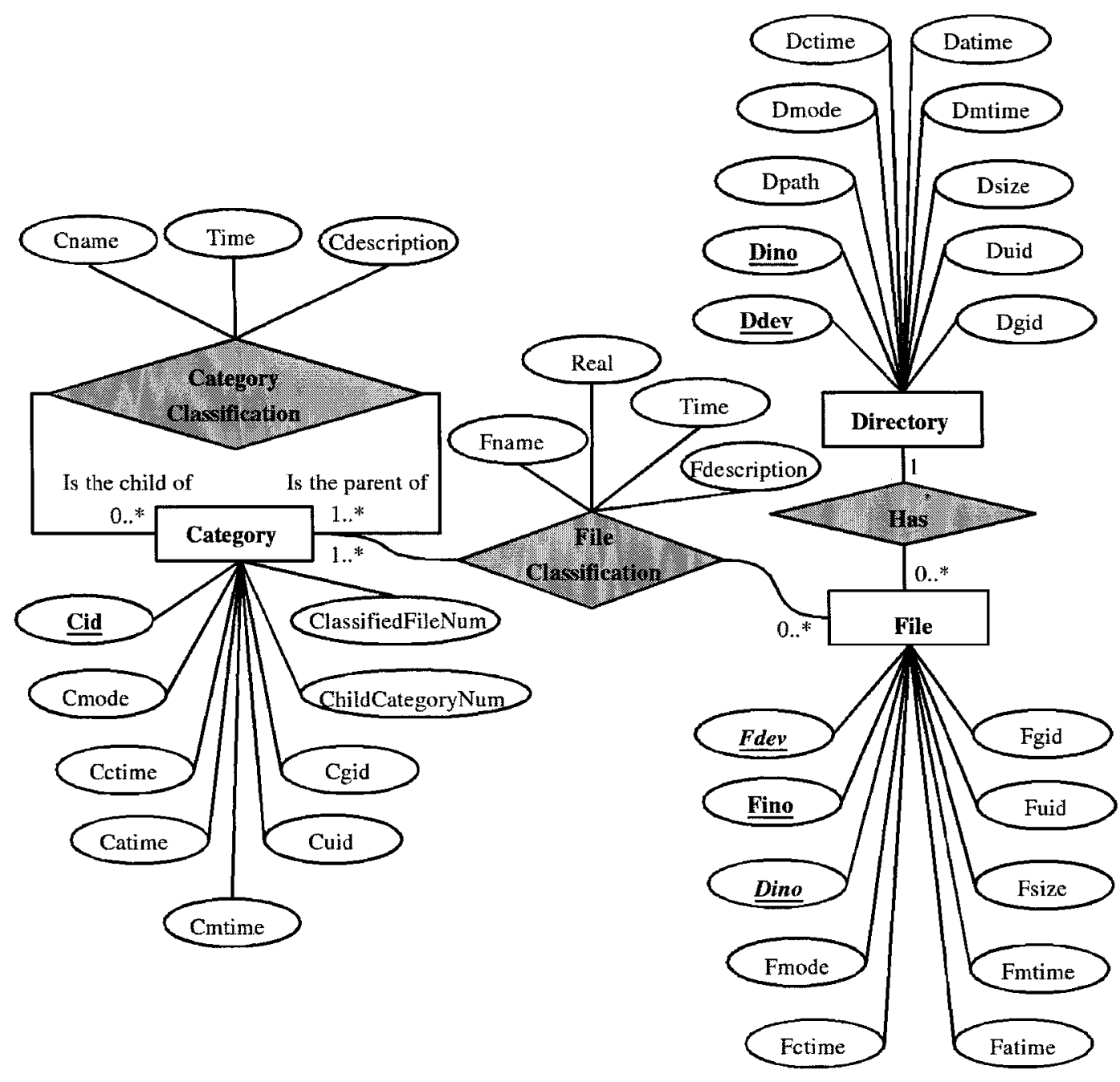

Figure 5.6: Entity relationship diagram 
Dino: Inode number of the directory that the file is under.

Fmode: Access and execution permission associated with the file.

Fctime: Creation time of the file.

Fatime: Time of the last file access.

Fmtime: Time of the last file modification.

Fsize: The size of the file.

Fuid: Owner id for the file.

Fgid: Group id for the file.

\section{- Directory}

It represents the concept of directory in the directory hierarchy, and consists of ten attributes:

Ddev: Device number of the directory.

Dino: Inode number of the directory.

Dmode: Access and execution permission associated with the directory.

Dctime: Creation time of the directory.

Datime: Time of the last directory access.

Dmtime: Time of the last directory modification.

Dsize: The size of the directory.

Duid: Owner id for the directory.

Dgid: Group id for the directory.

\section{Relationships:}




\section{- Category Classification}

It represents the relationship between categories. A category can classify one-tomany child categories, and at the same time be classified to zero-to-many parent categories (category $\mathbf{0}$ has no parent category). Category classification relationship has three attributes:

Cname: Category name.

Time: Creation time of the category name.

Cdescription: A short description of the relationship.

\section{- File Classification}

It represents the relationship between files and categories. A file can be classified to one-to-many categories, and a category can classify zero-to-many files. File classification has three attributes:

Fname: Logical file name.

Real: This field is used to identify if the logical file name is a real physical file name.

Time: Creation time of the logical file name.

Fdescription: A short description of the relationship.

\section{- Has}

It represents the relationship between files and directories. A directory has zero-tomany files, and a file can be placed in one-to-many directories.

\subsubsection{Logical Database Design}

Based on the conceptual database design, we convert the ER diagram into a relational database schema. Figure 5.7 displays the schema of all the tables in the CASH2 system. 
Figure 5.8 shows the data stored in them based on the sample category structure showed in Figure 4.2 .

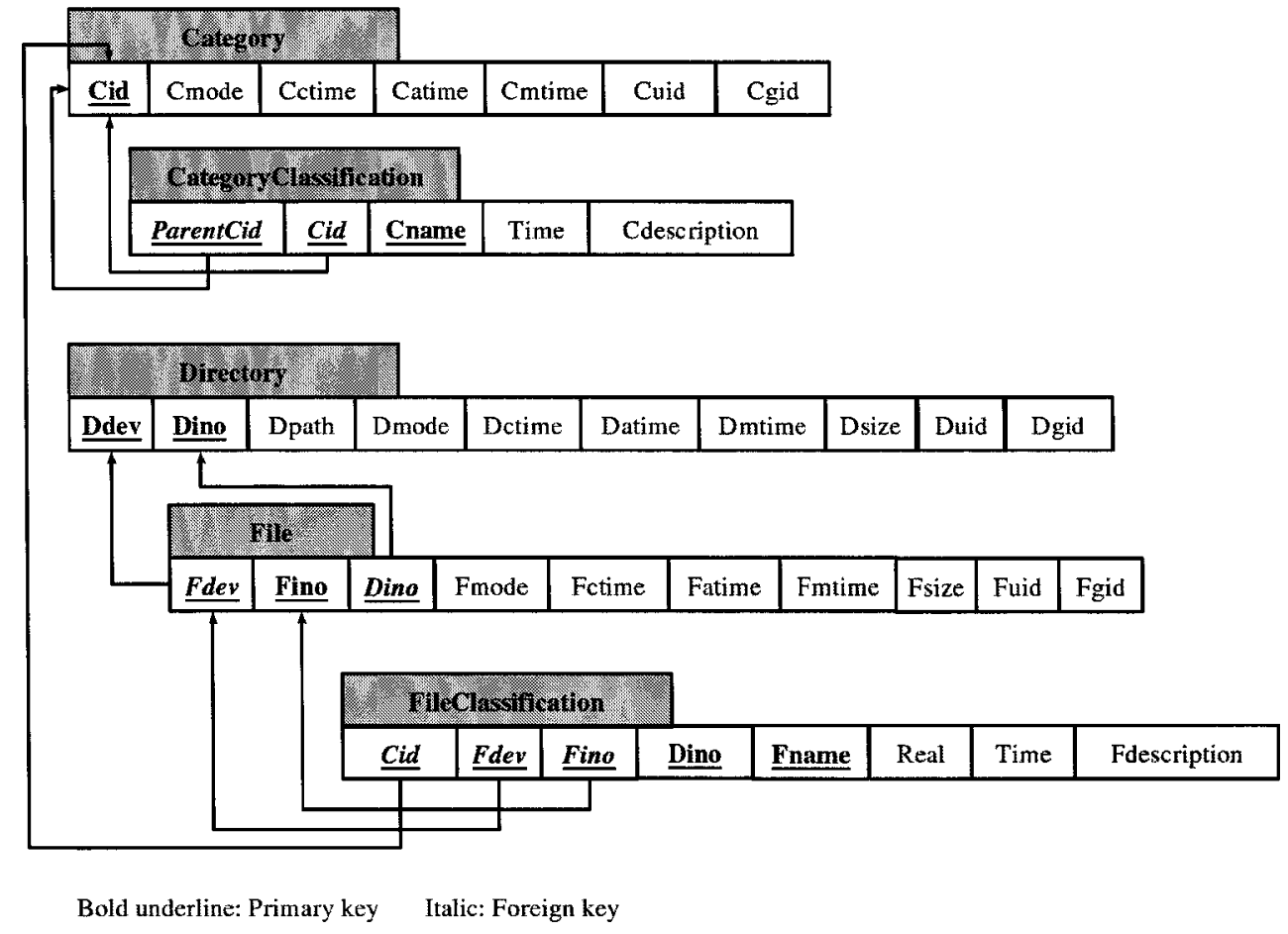

Figure 5.7: Database Schema

- The entity Category is mapped to the table Category. "Cid" is the unique numerical number that identifies the category, so it becomes the primary key in the table.

- The entity Directory is mapped to the table Directory. In a directory record, the combination of "Ddev" and "Dino" is unique, so it becomes the primary key in the table.

- The entity File is mapped to the table File. A file record is uniquely identified by the field "Fdev", "Fino", and "Dino". So the combination of these three attributes forms the primary key in the table. The relationship Has is mapped in this table as well. The primary key in the table Directory serves as the the foreign key in the table File. 
- The relationship Category Classification is mapped to the table CategoryClassification. The primary key in table Category is mapped as foreign keys in this table, and the combination of "ParentCid", "Cid" and "Cname" uniquely identifies a category classification record, and becomes the primary key in the table.

- The relationship File Classification is mapped to the table FileClassification. The primary keys in both table Category and File are mapped as the foreign keys in this table. The combination of "Cid", "Fdev", "Fino", "Dino" and "Fname" uniquely identifies a file classification relationship, and becomes the primary key in the table. 


Categor
\begin{tabular}{|c|c|c|c|c|c|c|}
\hline Cid & Cmode & Cctime & Catime & Cmtime & Cuid & Cgid \\
\hline 0 & 16877 & $2006-03-31$ & $2006-04-01$ & $2006-04-01$ & 1089 & 590 \\
\hline 1 & 16877 & $2006-03-31$ & $2006-04-01$ & $2006-04-01$ & 1089 & 590 \\
\hline 2 & 16877 & $2006-03-31$ & $2006-04-01$ & $2006-04-01$ & 1089 & 590 \\
\hline 3 & 16877 & $2006-03-31$ & $2006-04-01$ & $2006-04-01$ & 1089 & 590 \\
\hline$\ldots$ & $\ldots$ & $\ldots$ & $\ldots$ & $\ldots$ & $\ldots$ & $\ldots$ \\
\hline
\end{tabular}

Cateblydassification
\begin{tabular}{|c|c|c|c|c|}
\hline ParentCid & Cid & Cname & Time & Cdescription \\
\hline 0 & 1 & Beverage & $2006-04-01$ & Null \\
\hline 1 & 2 & NonAlcoholic & $2006-04-01$ & Null \\
\hline 1 & 3 & Carbonated & $2006-04-01$ & Null \\
\hline$\ldots$ & $\ldots$ & $\ldots$ & $\ldots$ & $\ldots$ \\
\hline
\end{tabular}

File
\begin{tabular}{|c|c|c|c|c|c|c|c|c|c|}
\hline Fdev & Fino & Dino & Fmode & Fctime & Fatime & Fmtime & Fsize & Fuid & Fgid \\
\hline 8388736 & 102272 & 102133 & 33188 & $2006-04-01$ & $2006-04-01$ & $2006-04-01$ & 27610 & 1089 & 590 \\
\hline 8388736 & 102273 & 102136 & 33188 & $2006-04-01$ & $2006-04-01$ & $2006-04-01$ & 2923 & 1089 & 590 \\
\hline 8388736 & 102274 & 102139 & 33188 & $2006-04-01$ & $2006-04-01$ & $2006-04-01$ & 896 & 1089 & 590 \\
\hline$\ldots$ & $\ldots$ & $\ldots$ & $\ldots$ & $\ldots$ & $\ldots$ & $\ldots$ & $\ldots$ & $\ldots$ & $\ldots$ \\
\hline
\end{tabular}

Fleclassfreation
\begin{tabular}{|c|c|c|c|c|c|c|c|}
\hline Cid & Fdev & Fino & Dino & Fname & Real & Time & Fdescription \\
\hline 2 & 8388736 & 102273 & 102136 & PerrienMineralWater & y & $2006-04-01$ & Null \\
\hline 3 & 8388736 & 102273 & 102136 & PerrierMineralWater & y & $2006-04-01$ & Null \\
\hline 9 & 8388736 & 102272 & 102133 & LiptonHerbalTea & y & $2006-04-01$ & Null \\
\hline$\ldots$ & $\ldots$ & $\ldots$ & $\ldots$ & $\ldots$ & $\ldots$ & $\ldots$ & $\ldots$ \\
\hline
\end{tabular}

Directery
\begin{tabular}{|c|c|c|c|c|c|c|c|c|c|}
\hline Ddev & Dino & Dpath & Dmode & Dctime & Datime & Dmtime & Dsize & Duid & Dgid \\
\hline 8388736 & 102133 & /usr/qwang/Tea & 16877 & $2006-03-22$ & $2006-03-22$ & $2006-03-22$ & 512 & 1089 & 590 \\
\hline 8388736 & 102134 & /usr/qwang/Coffee & 16877 & $2006-03-22$ & $2006-03-22$ & $2006-03-22$ & 512 & 1089 & 590 \\
\hline 8388736 & 102135 & /usr/qwang/Cola & 16877 & $2006-03-22$ & $2006-03-22$ & $2006-03-22$ & 512 & 1089 & 590 \\
\hline$\ldots$ & $\ldots$ & $\ldots$ & $\ldots$ & $\ldots$ & $\ldots$ & $\ldots$ & $\ldots$ & $\ldots$ & $\ldots$ \\
\hline
\end{tabular}

Figure 5.8: Example Of Tables 


\section{Chapter 6}

\section{IMPLEMENTATION AND SYSTEM MEASUREMENT}

In the prior chapters, we mentioned that the previous work of CASH did not correctly implement our multiple hierarchical categorization structure, and the system had no means to measure its performance. In this chapter, we aim to improve our work in the corresponding aspects to achieve our objectives (6), (7), and (8) (on page 9).

\subsection{Operations}

We divide all the operations in $\mathrm{CASH} 2$ into three categories, basic operations, category and file operations, and other operations. We discuss the details in the following sections.

\subsubsection{Basic Operations}

The basic operations are fundamental to all the other operations, which serves as the groundwork of CASH2. In CASH2, we have two basic operations: directed cycle detecting and path computing. 


\subsubsection{Directed Cycle Detecting}

This operation is used to check whether there is a directed cycle existing in the multiple hierarchical categorization structure to guarantee the correctness of the structure. To determine if there is a cycle between a category node End and its parent category node Begin, we conduct a breadth first search in the steps shown in Figure 6.1.

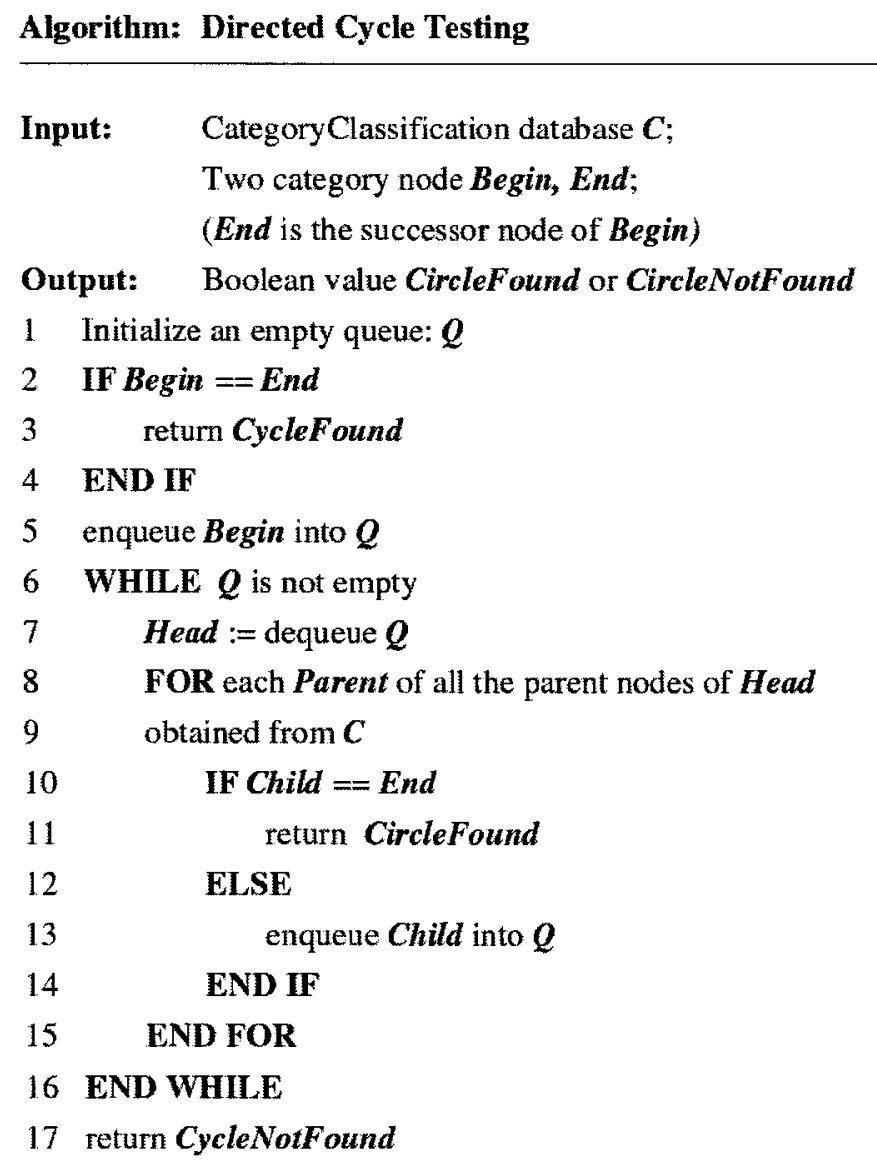

Figure 6.1: Algorithm of Directed Cycle Detecting

To give an example, as shown in Figure 6.2, we intend to classify the category 10 to the category $\mathbf{1 5}$ with the name "f", i.e., to add a directed edge in our category DAG from 15 to 10 with edge name $f$. Before we conduct this operation, we need to check if 
adding this edge will create a cycle within our structure. First, we examine if 15 is equal to 10 . Since the result is negative, we add 15 into an empty queue $Q$. Then, we dequeue 15 and examine its parent node ( the parent node information obtained by querying on the CategoryClassification database), 12. Because 12 does not equal to 15 , we enqueue 12 into $Q$. To continue, we dequeue 12 , and examine its parent node, here we find 10 , which means a cycle is detected, then we return "A cycle is found". As a result, the edge $f$ cannot be added, i.e., the category $\mathbf{1 0}$ cannot be classified to the category $\mathbf{1 5}$.

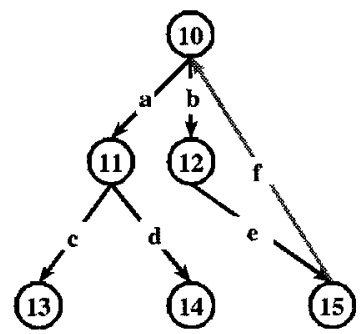

Figure 6.2: Example: Directed Cycle

\subsubsection{Path Computing}

The operation is used to compute category paths. Its purpose is to display all the pathrelated information in CASH2.

Top-To-Bottom Path Computing To find all the path information from a category node Begin downwards, we conduct a depth first search. The algorithm is shown in Figure 6.3.

To give an example, as shown in Figure 6.4, we intend to find out all the path information starting at the root category $\mathbf{0}$. First, we create a temporary Berkeley DB database $T$, and insert all the records in CategoryClassification database that satisfy "ParentCid $==0$ " into it, this means we insert edge $a$ and $b$ int $T$. Then, we add 0 into an empty stack $S$, and check if 0 is a leaf category node. Since the result is negative, we get one unprocessed edge from $T$ with 0 as the parent node, i.e., the edge a, and push $a$ and 2 into $S$, at the same time, 


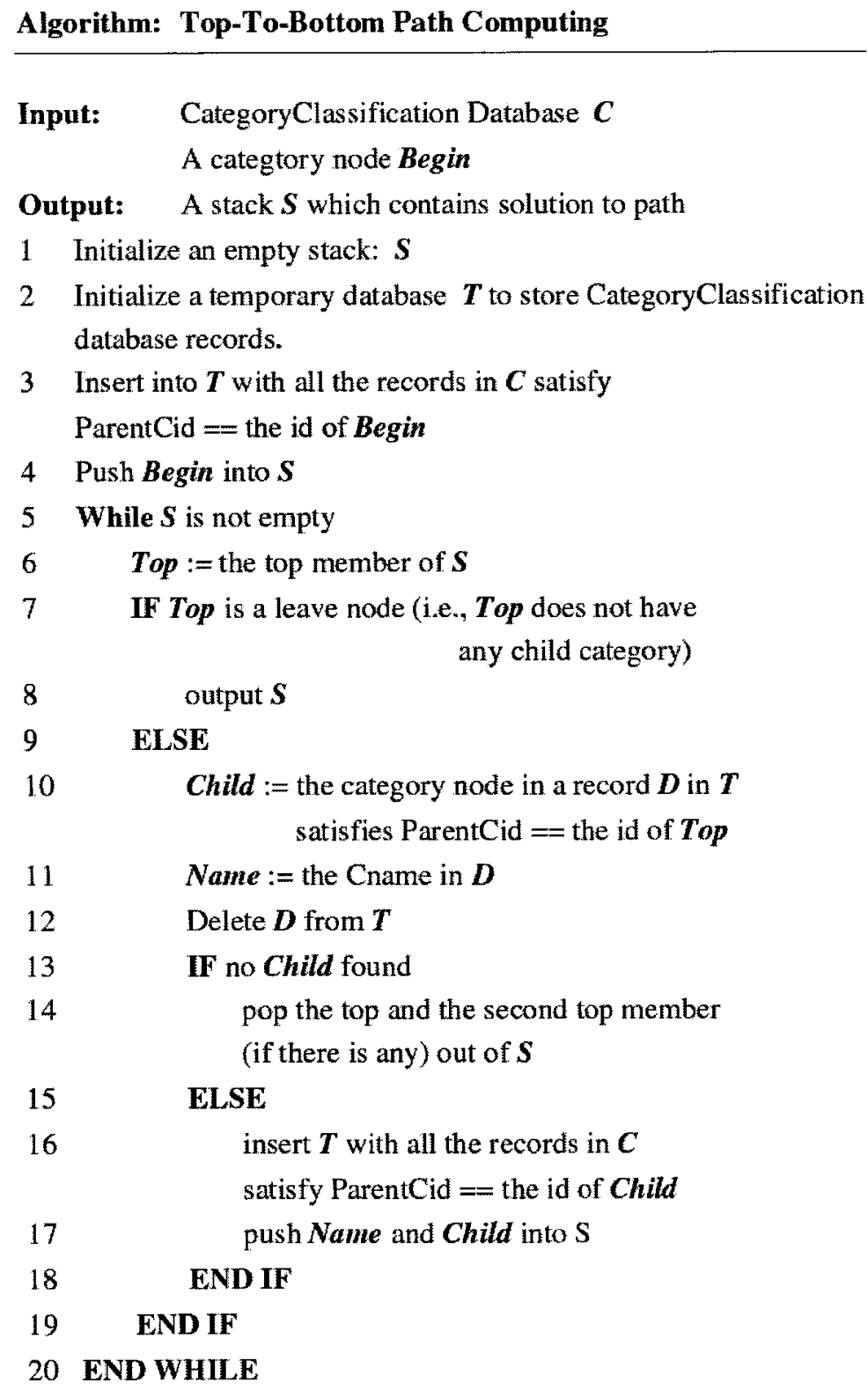

Figure 6.3: Algorithm of Top-To-Bottom Path Computing 
we delete the record of the edge $a$ from T. In this way, we mark the edge $a$ as a processed edge. For now, the data in $S$ from bottom to top, is “ 0 , a, 2 ", and the unprocessed edges left in $T$ is " $b$ ". To continue our depth first search approach, we check if 2 is a leaf node. Since the result is positive, we output, the first time, the path information contained in $S$, i.e., "/ a". Meanwhile, we pop out "2" and "a" from $S$.

To continue, we get the top member in $S$, i.e., " 0 " and check if any unprocessed edge with 0 as the parent node is left in $T$, and we find the edge $b$. Then we push $b$ and 1 into $S$, at the same time, we delete the record of the edge $b$ from $T$. Then, we insert all the edges that have 1 as their parent node into $T$, i.e., we insert edge $c$ and $d$ into $T$. For now, the data in $S$, from bottom to top, is " $0, \mathrm{~b}, 1$ ", and the unprocessed edges left in $T$ is "c" and " $\mathrm{d}$ ". To continue, we check that $S$ is not empty, we get the top member in $S$, i.e., "1". Since 1 is not a leaf node, we get one of the unprocessed edges left in $T$ that have 1 as their parent node, i.e., the edge $c$, and push $c$ and 2 into $S$, at the same time, we delete the record of the edge $c$ from $T$. Since 2 does not have any child node, no edge is added into $T$ in this round. For now, the data in $S$ from bottom to top, is “ $0, \mathrm{~b}, 1, \mathrm{c}, 2$ ", and the unprocessed edges left in $T$ is "d". To continue, we get the top member of $S$, i.e., 2 , and check if 2 is a leaf node, and the result is positive. So we output, the second time, the path information contained in $S$, i.e., "/ b/ c". Meanwhile, we pop out "2" and "c" from $S$.

We continue our depth first search again by getting the top member in $S$, i.e., "1" and check if any unprocessed edge with 1 as the parent node is left in $T$, and we find the edge $d$. Then, we push $d$ and 3 into $S$. In the meantime, we delete the record of the edge $d$ from $T$. Since 3 does not have any child node, no edges is added into $T$ in this round. For now, the data in $S$ from bottom to top, is " $0, \mathrm{~b}, 1, \mathrm{~d}, 3$ ", and there is no unprocessed edges left in $T$. To continue, we check that $S$ is not empty, then we get the top member in $S$, i.e., "3", and check if it is a leaf node. Since the result is positive, we output, the third time, the path information contained in $S$, i.e., "/ b/ d". Meanwhile, we pop out "3" and "d" from $S$.

To finish, we check again that $S$ is not empty, so we get the top member "1". Since 1 
has child nodes, however there is no unprocessed edges left in $T$, we pop out " 1 " and "b" from $S$. Then, we continue to check and find that only " 0 " is left in $S$. Since 0 has child nodes, but there is no unprocessed edges left in $T$, we pop out " 0 " from $S$. Now $S$ is empty and our procedure ends.

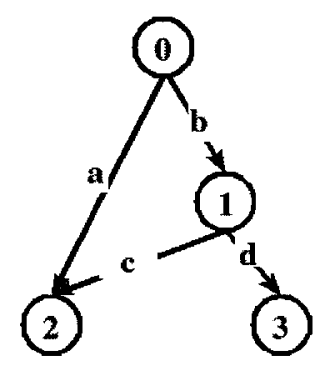

Figure 6.4: Example: Path Computing

Bottom-To-Top Path Computing To find all the path information from a category node End upwards, we conduct a depth first search backwards. The algorithm is shown in Figure 6.5 .

We still use Figure 6.4 to demonstrate this example. This time, our intent is to find out all the path information end at the category 2 . First, we create a temporary Berkeley DB database $T$, and insert all the records in CategoryClassification database that satisfy "Cid $==2$ " into it. This means we insert all the edges that have 2 as their child node into $T$, i.e., the edge $a$ and $c$. Then, we add 2 into an empty stack $S$, and check if 2 is the root category node. Since the result is negative, we get one unprocessed edge from $T$ with 2 as the child node, i.e., the edge $a$, and push $a$ and 0 into $S$, meanwhile, we delete the record of the edge $a$ from $T$. In this way, we mark the edge $a$ as a processed edge. Since 0 does not have any parent node, no edge is added into $T$ in this round. For now, the data in $S$, from bottom to top, is " 0 , a, 2 ", and the unprocessed edges left in $T$ is "c". to continue, we get the member of $S$, i.e., 0 , and check if 0 is the root category node. Since the result is positive, we output, the first time, the path information contained in $S$, i.e., "/ a". Meanwhile, we pop out " 0 " 


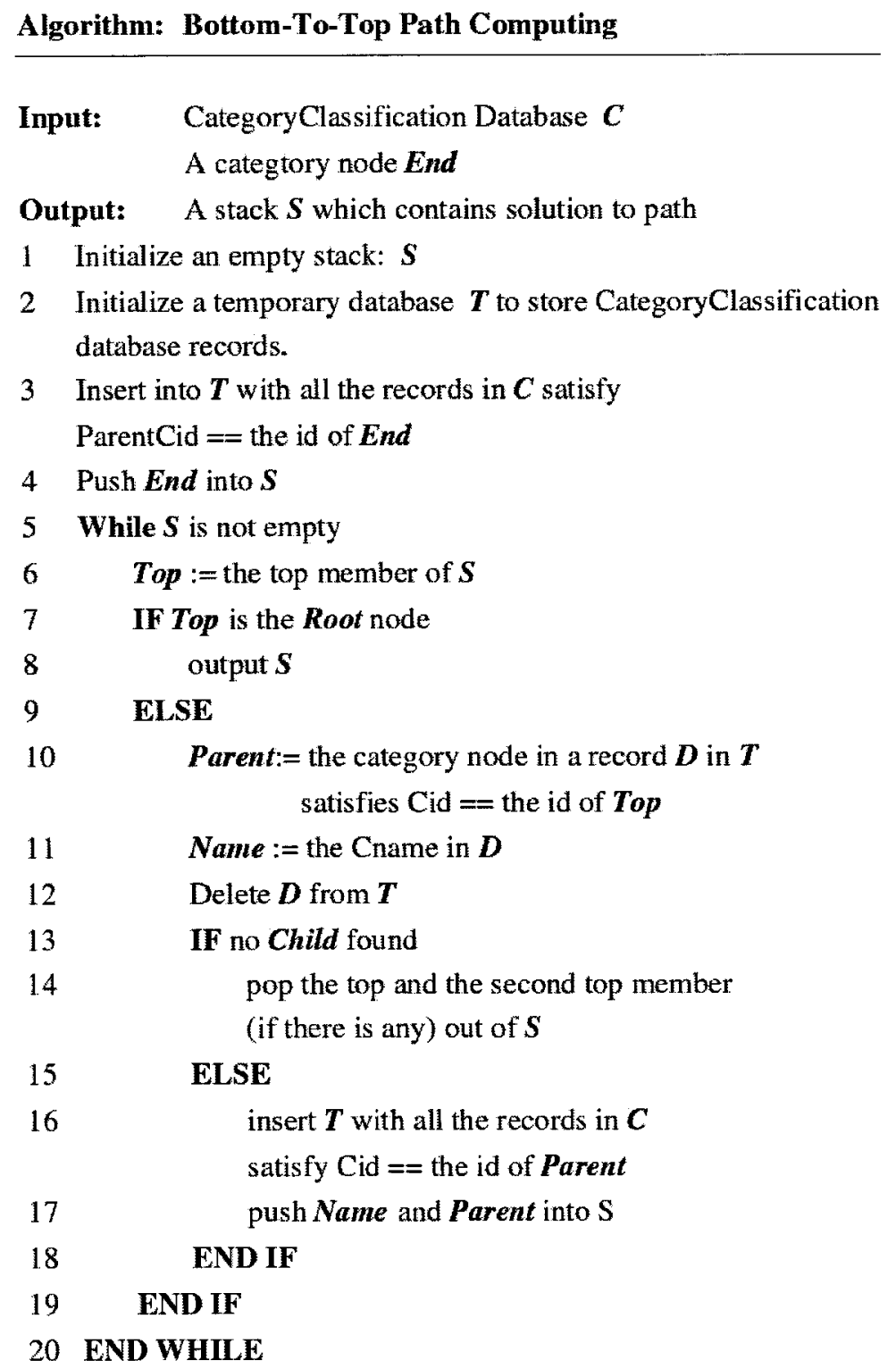

Figure 6.5: Algorithm of Bottom-To-Top Path Computing 
and "a" from $S$.

To continue, we get the top member in $S$, i.e., " 2 ", and check if there are any unprocessed edge with 2 as the child node is left in $T$, we find the edge $c$. Then, we push $c$ and 1 into $S$, at the same time, we delete the record of the edge $c$ from $T$. Since 1 has parent nodes, we insert all the edge that have 1 as their node into $T$, i.e, we insert the edge $b$ into $T$. For now, the data in $\mathrm{S}$, from top to bottom, is " $1, \mathrm{c}, 2$ ", and the unprocessed edges left in $T$ is $b$. To continue, we check that $S$ is not empty, then we get the top member in $S$, i.e., "1", since 1 is not the root node, we get the unprocessed edge left in $T$ that has 1 as its child node, i.e., the edge $b$, and push $b$ and 0 into $S$, at the same time, we delete the record of the edge $b$ from $T$. Since 0 does not have any parent node, no edge is added into $T$ in this round. For now, the data in $S$, from top to bottom, is " $0, \mathrm{~b}, 1, \mathrm{c}, 2$ ", and there is no unprocessed edges. to continue, we get the top member of $S$, i.e., 0 , and check if 0 is the root node. Since the result is positive, we output, the second time, the path information contained in $S$, i.e., "/ b/ $c$ ". Meanwhile, we pop out " 0 " and "b" from $S$.

To finish, we check again that $S$ is not empty, so we take the top member "1". Since 1 has parent nodes, but, there is no unprocessed edges left in $T$, we pop out " 1 " and "c" from $S$. We continue to check and find that only " 2 " is left in $S$. Since 2 has parent nodes, however there is no unprocessed edges left in $T$, we pop out " 2 " from $S$. Now, $S$ is empty and our procedure ends.

\subsubsection{Category and File Operations}

In CASH2, we implement category and file operations with Unix/Linux like commands (See appendix for the detail). The syntax of commands in CASH2 is similar to that in $\mathrm{CASH}$. Because of the improved design in $\mathrm{CASH} 2$, we have rewritten the implementation program to realize the correct category structure. Additionally, we add more options into the existing command set to realize some features in $\mathrm{CASH} 2$ that did not get implemented 
in CASH. In this section, we explain some of the major category and file related operations, such as search, add, and remove.

First, we would like to introduce several basic terms that are used when we work on the multiple hierarchical categorization structure. The working category means the current category a user is working with. The utility command cdcat allows the user to move around in the category structure to reach the working category. The working category path means the category path that the user chooses to work under. For example, as shown in Figure 6.6, the command cdcat /b/e changes the current working category to 5 , and sets the working path to "/ $\mathrm{b} / \mathrm{e}$ ", and the working category name becomes $e$. In CASH2, the user can only choose one path to work under. The parallel edge $f$ of $e$ is called the self alias category name of the working category name. The path "/ b/ f" is called the self alias category path. The edge $d$ and $g$ are called the non-self alias category name of the working category name. The category path "/ a/ d" or "/ c/ g" is called the non-self alias category path.

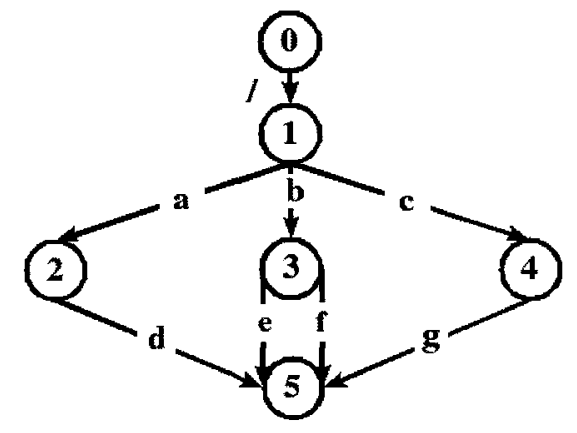

Figure 6.6: Working Category and Aliases

In the following content, unless otherwise specified, we will be using Figure 4.2 (on page 43) to illustrate our example. 


\subsubsection{Category-based Search Operations}

These types of operation are used to find category and file information based on our multiple hierarchical category structure by querying related databases in the system. In CASH2, we use lscat and cdcat to implement them.

\subsection{Command: 1scat}

The following shows the command syntax.

lscat [option] [target]

Options: none: List the content of the target category, including all the category classification and file classification information with the target as the parent category.

-w: Displays the target category (a wild card matching on category name is enabled) information in the system.

-1 : Displays in long form.

$-\mathrm{R}$ : Recursively displays all category and file information in the sub-DAG rooted at the target category.

$-s$ : Displays the self-information of the target category.

$-i$ : Displays the alias information of the target category.

$-\mathrm{P}$ : Displays all the path information in the sub-DAG rooted at the target category.

$-\mathrm{C}$ : Displays all the path information from root to the target category.

$-\mathrm{p}$ : Gives uses options to choose a parent category of the target category.

$-\mathrm{f}$ : Displays the target file information under its specified category.

$-\mathrm{F}$ : Displays the target file information under its predecessor category.

\section{Option: - w}

The command lscat - a displays the target category information in the system. A wild card matching on category name is enabled. The target is in a single category name form. 
Example(s):

\begin{tabular}{|c|c|c|c|}
\hline Total Category & Name (s): 21 & & \\
\hline 1 & Caffeinated/ & Cola/ & MadeFromGrape/ Wine/ \\
\hline Alcoholic/ & Champagne/ & Cola/ & NonAlcoholic/ \\
\hline Beer/ & Champagne/ & HervalTea/ & RedWine/ \\
\hline Beer/ & Coffee/ & Hot/ & Carbonated/ \\
\hline Beverage/ & Coffee/ & MadeFromGrain/ & Whitewine/ \\
\hline
\end{tabular}

Explanation:

1. This line shows the total number of category names.

2. This line and the following lines show all the category names in alphabetical order.

\section{Option: -1}

The command Iscat -1 displays all the information in long form.

\section{Example(s):}

We combine -1 with - w option to display the category name information in long form.

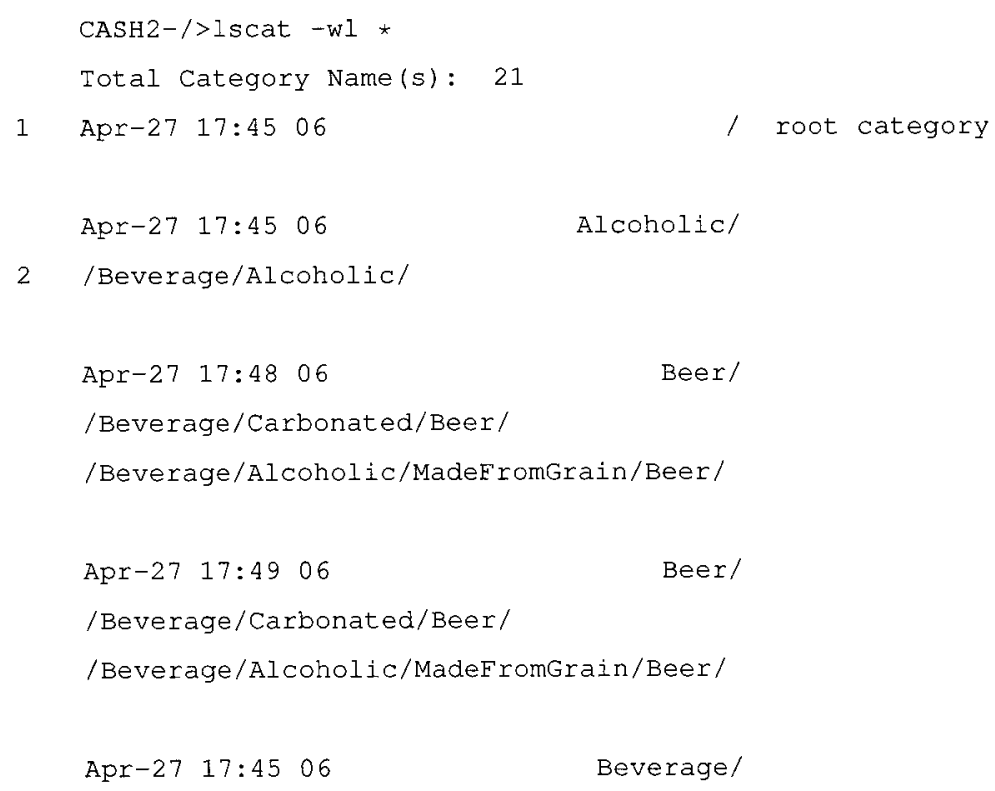




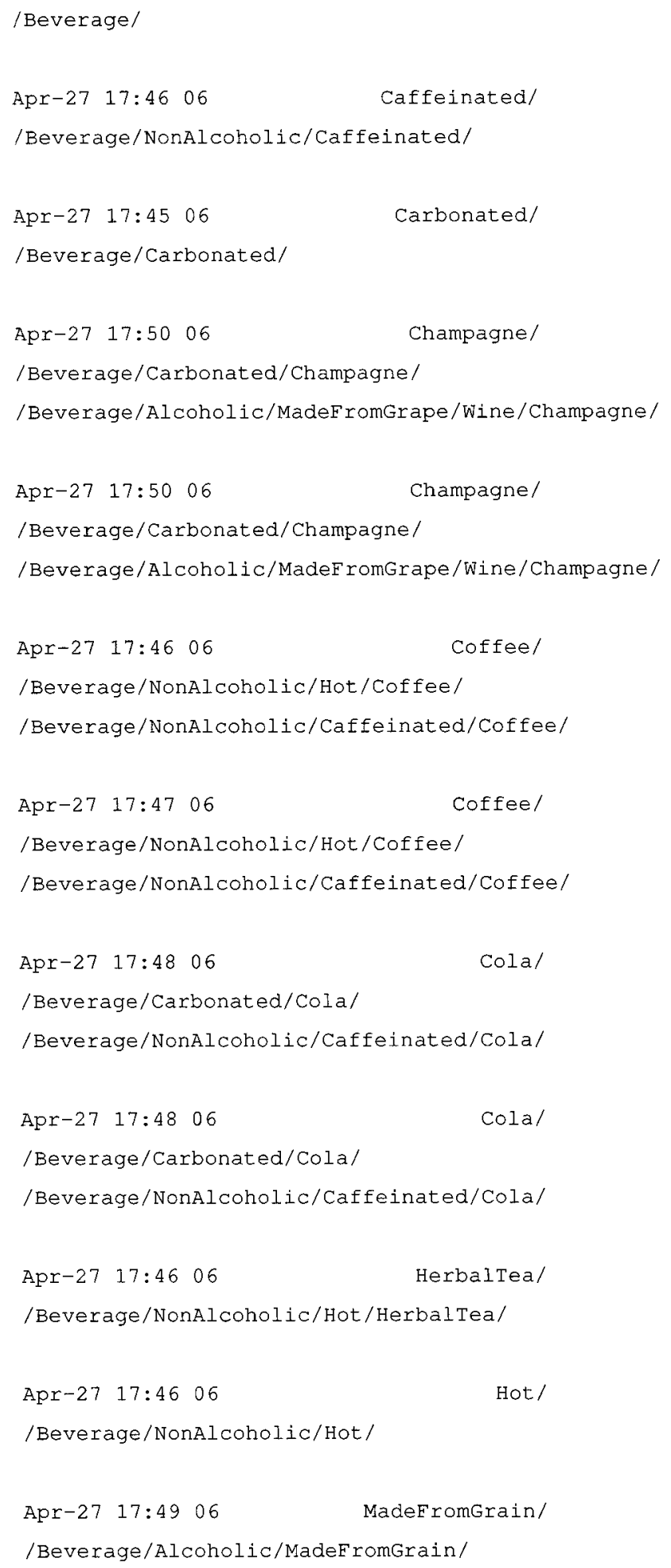




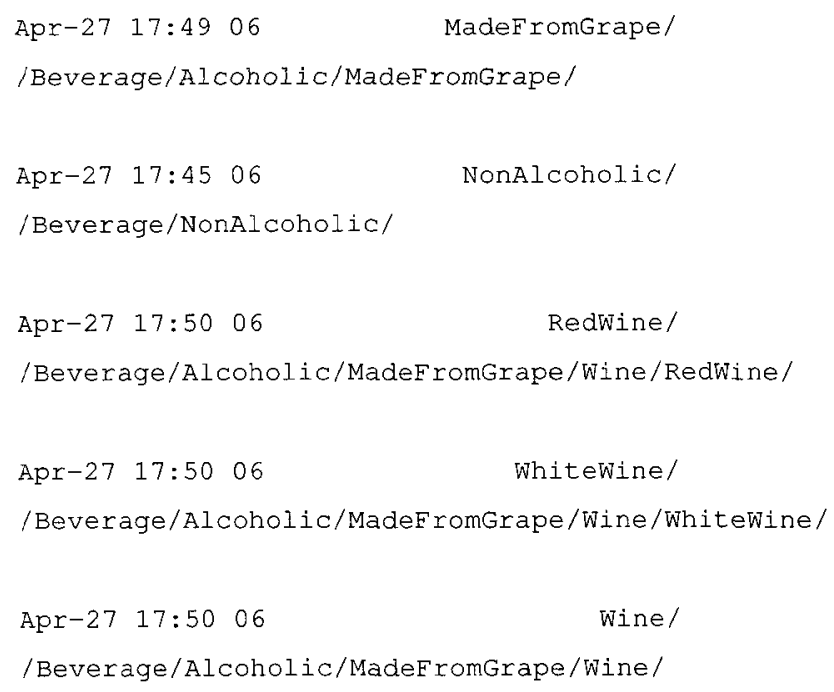

\section{Explanation:}

1. The first column shows the creation time of a category classification, the second column shows the category names, and the third column shows a short description of the category classification, if there is any.

2. This line shows the path information of the displayed category name (multiple lines are required if the category has multiple paths).

\section{Option: $-\mathbf{R}$}

The command lscat $-\mathrm{R}$ displays, recursively, the categories and file information in the sub-DAG which is rooted at the target category.

\section{Example(s):}

We would like to display the category and file information in the sub-DAG rooted at 1 with name "Alcoholic", as shown in Figure 6.7.

1 CASH2-/>lscat $-\mathrm{R}$ Alcoholic

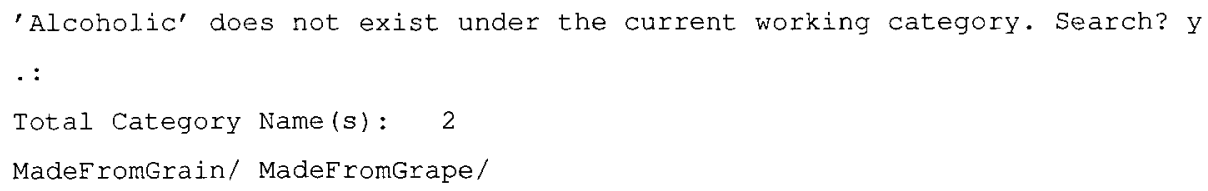




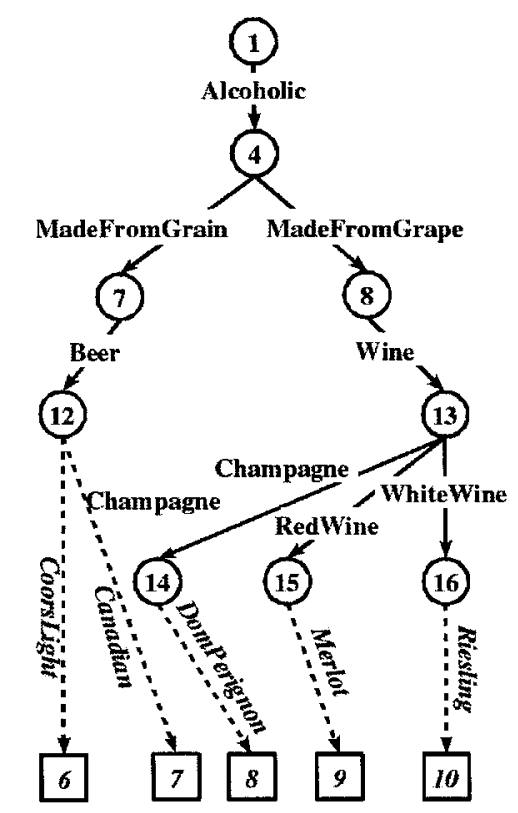

Figure 6.7: Example: Sub-DAG Rooted at "Alcoholic"

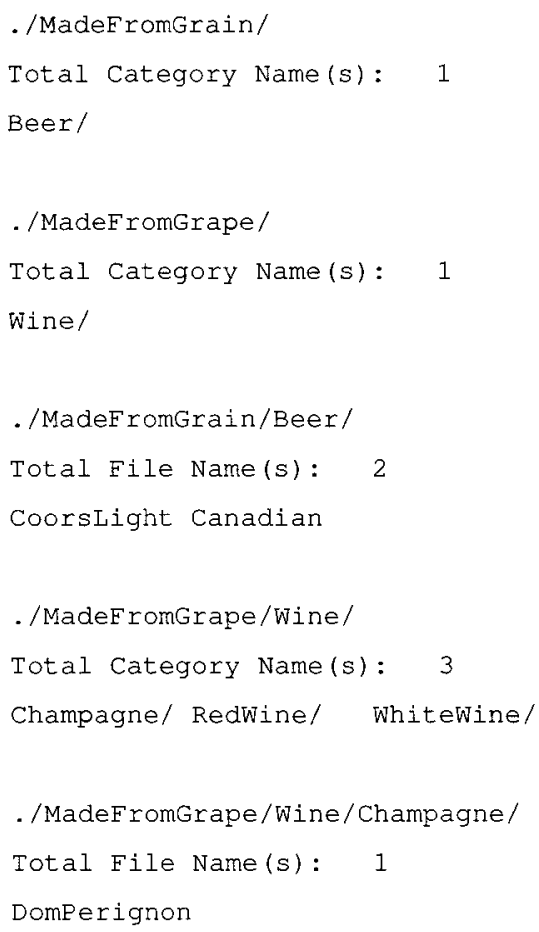




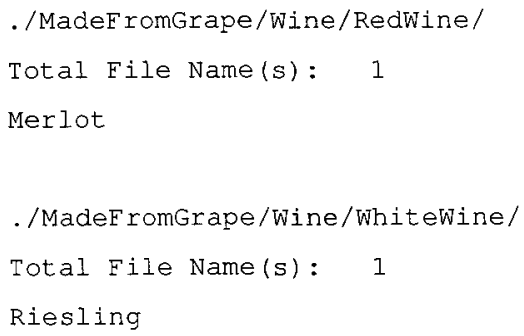

Explanation:

1. The command lscat $-\mathrm{R}$ Alcoholic is meant to display all the information in the sub-DAG which is rooted at the target category "Alcoholic". Because it is not under the current working category $\mathbf{0}$, the system will ask if users want to search for it in the wholecategory structure. After we answer "yes", the result is displayed recursively.

\section{Option: $-s$}

This Iscat - s command displays the current working category information itself. Example(s):

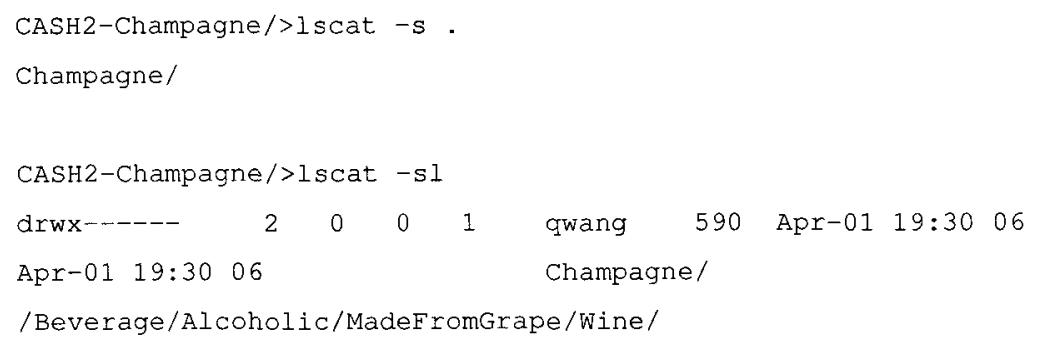

Explanation:

1. The category prompt shows that the current working category is "Champagne". The lscat $-s$. command is meant to display the self-information of the input category ". ", which means the current working category 5 with name "Champagne".

2. This line displays the name of the category 15 .

3. The $-\mathrm{s}$ option combined with - I option is used to display the self-information of 
the target category with name "Champagne" in a long form.

4. This line displays the general information about the category $\mathbf{1 5}$, from left to right, the access mode, the number of its name(s), the number of the category name(s) with regard to the category $\mathbf{1 5}$ as the parent category, the number of self-alias category name(s), the number of non-self alias category name(s), the ownership, the group ownership, and the creation time.

5. This line shows the information of the working category name "Champagne", from left to right, the creation time of the file classification, and the file logical name.

6. This line shows the working category path that the user has used to reach category 15.

\section{Option: $-i$}

The lscat -i command displays all the alias category names or path information. It is an extended command option which is not included in the CASH command design.

Example(s):

The following command-line example shows the alias information of the category $\mathbf{1 0}$ in Figure 6.8 .

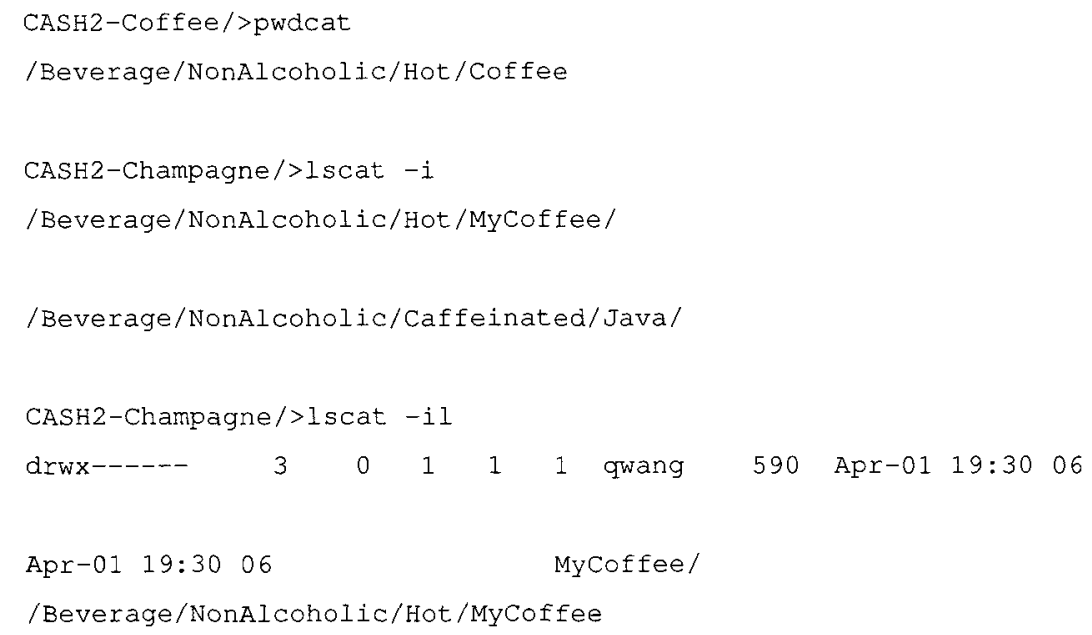




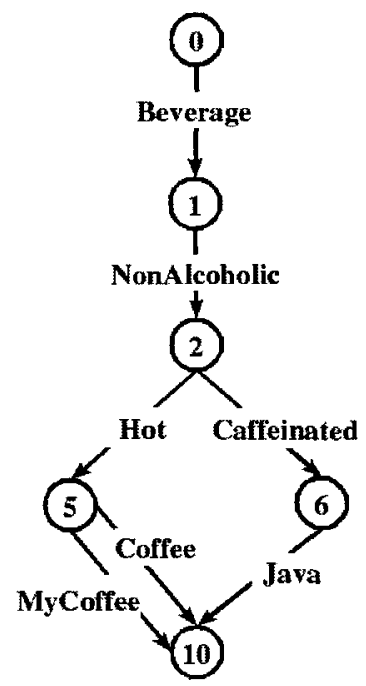

Figure 6.8: Example: 1scat -i

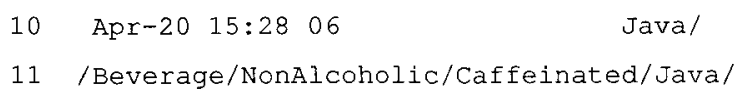

\section{Explanation:}

1. the pwdcat is used to display the working category path information.

2. This line shows that we are currently working under "/ Beverage/ NonAlcoholic/ Hot/ Coffee".

3. Iscat $-i$ is meant to display the alias information of "Coffee".

4. This line displays the category path information of the self alias category, "MyCoffee".

5. This line displays the category path information of the non-self alias, "Java".

6. the $-i$ option combined with -1 option is used to display the alias category information in long form.

7. This line displays the general information about the category $\mathbf{1 5}$, from left to right, the first is the access mode, the second is the number of its name(s). Here, category 
$\mathbf{1 5}$ has three category names, "Coffee", "MyCoffee", and "Java", therefore, the number shown here is shown as 3 . The next number is the number of the category name(s) with regard to the category $\mathbf{1 5}$ as the parent category, and since category $\mathbf{1 5}$ does not have any child category, this number is shown as 0 . The next number is the number of self alias category name(s), and category $\mathbf{1 5}$ has one self alias name "MyCoffee", therefore, the number is shown as 1 . Next, is the number of non-self alias category name(s). Category 15 has one non-self alias name "Java", therefore, the number is shown as 1 . The next number is the number of files classified to category $\mathbf{1 5}$, and we have 1 here. Continue on, is the ownership, the group ownership, and the creation time of category 15.

8. This line displays the information of the self alias category name "MyCoffee". The first column is the creation time of this category classification, and the second column is the category name.

9. This line displays the category path information of the self alias, "MyCoffee". 10. this line displays the information of the non-self alias category name "Java". The first column is the creation time of the category classification, and the second column is the category name.

11. This line displays the category path information of the non-self alias, "Java".

\section{Option: $-\mathrm{P}$}

The lscat $-\mathrm{P}$ command displays all the category path(s) information rooted at the target category. It is an extended command option which is not included in the CASH command design.

Example(s):

CASH2-/>lscat $-\mathrm{P}$ Alcoholic

'Alcoholic' does not exist under the current working category. Search? y

1 /MadeFromGrain/Beer/ 
/MadeFromGrape/Wine/Champagne/

/MadeFromGrape/Wine/RedWine/

/MadeFromGrape/Wine/Whitewine/

Explanation:

1. After we issue the command lscat $-\mathrm{P}$ Alcoholic, the result shows four paths in the sub-DAG that is rooted at the category $\mathbf{4}$ with the name "Alcoholic".

\section{Option: $-\mathrm{C}$}

The lscat $-\mathrm{P}$ command displays all the category path(s) information from root to the target category.

Example(s):

Explanation:

1. After we issue the command lscat $-\mathrm{C}$ Coffee, the result shows two paths information of category $\mathbf{1 0}$.

\section{Option: $-p$}

The lscat $-p$ command gives users options to choose a parent category for displaying information. It is an extended command option which is not included in the CASH command design.

Example(s):

1 CASH2 $-/>$ lscat $-p$ Beer 


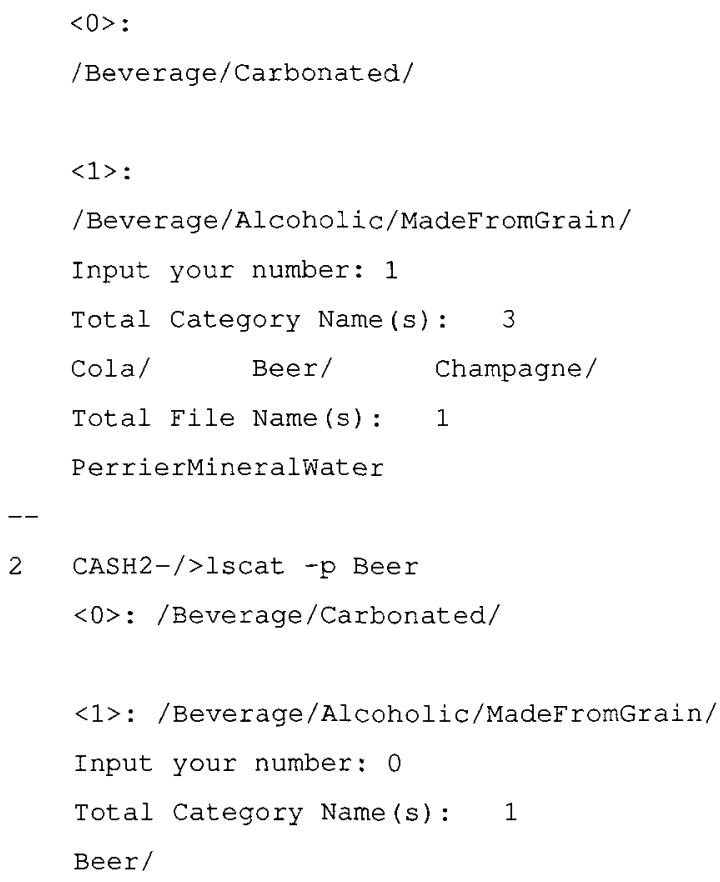

Explanation:

1. The command lscat -P Beer gives two parent category path options of category $\mathbf{1 2}$ for users to choose one to display information, and we choose the second option, then the system displays the categories and file information classified under category 3.

2. In this example, we choose the first parent path option, then the system displays the categories and file information classified under category 7.

\section{Option: $-\mathbf{f}$}

The lscat $-f$ command displays the target file information under its parent category. The format of the command is as follows:

lscat - $f$ [file logical name in category form]

Example(s):

1 CASH $2-/>1$ scat $-f$ *

CASH $2-/>$ 
CASH2 - / > Iscat -f Beer/*

'Beer' does not exist under the current working category. Search? y

/Beverage/Carbonated/Beer/CoorsLight

/Beverage/Alcoholic/MadeFromGrain/Beer/CoorsLight

/Beverage/Carbonated/Beer/Canadian

/Beverage/Alcoholic/MadeFromGrain/Beer/Canadian

Explanation:

1. Iscat $-f \star$ is meant to display all the file information classified under the current working category (i.e., the root category). The result shows there is no files directly classified to root.

2. Iscat $-f$ Beer $/ *$ is meant to display all the file information classified under the category with the name "Beer". The system displays all the file names in their category path form.

10. Option: $-\mathbf{F}$

The lscat $-\mathrm{F}$ command displays all the target file information under its predecessor category. Further more, it enables logical operations on sets of file information obtained from different sub-DAGs. the logical operations includes OR, AND and NOT. In $\mathrm{CASH} 2$, we use $+{ }^{\wedge}{ }^{\wedge}$ and - to represent them. The $-\mathrm{F}$ option in lscat command is an extended command option, which is not included in CASH design.

The format of the command is as follows:

lscat [logical operator]

[file logical name in category form]

Example(s):

1 CASH2-/>1scat $-F$ *

/Beverage/Carbonated/Beer/Canadian

/Beverage/Alcoholic/MadeFromGrain/Beer/Canadian 


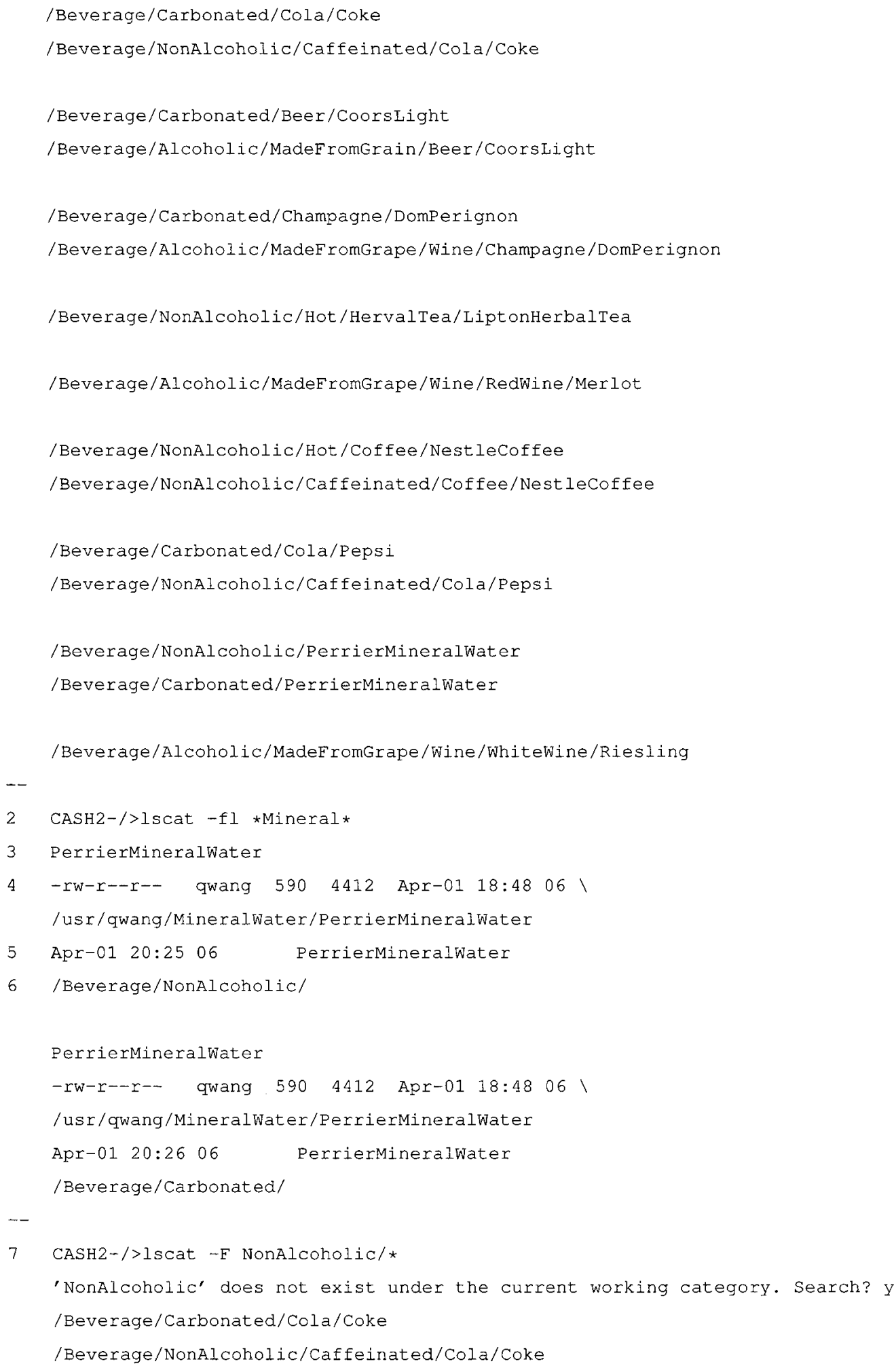




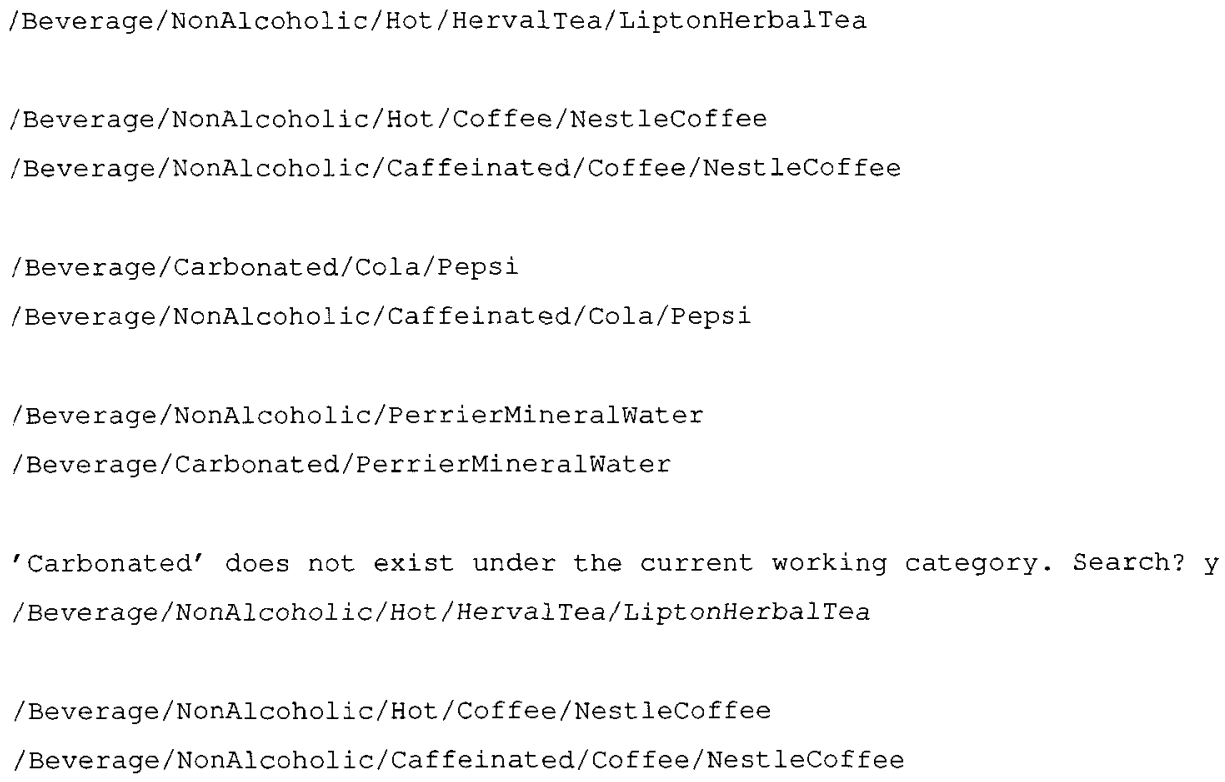

Explanation:

1. The Iscat $-F *$ command displays all the file classifications information. * represents a wild card matching against the logical file names. The results are formatted as a logical file name followed by its category path(s) information.

2. The lscat $-F l \star M i n e r a l *$ command displays all the file classifications information in long form with the logical file name(s) that matches *Mineral*.

3. This line shows the matching logical file name "PerrierMineralWater".

4. This line shows the physical file information, from left to right, the access mode, the ownership, the group ownership, the file size, the creation time, and the directory path.

5. This line shows the logical file name, and the creation time of it.

6. This line displays the category path information for the file "PerrierMineralWater".

7. The lscat $-F$ NonAlcoholic/* command displays all the file information under category 2 and all its descendants, i.e., 5, 6, 9, 10 and 11 . As a result, all the 
logical file names in category path form are displayed.

8. The lscat $-\mathrm{F}$ NonAlcoholic/* - Carbonated/* command displays all the file information under category 2 and its descendants, excluding those who are under category 3 and its descendants.

\subsection{Command: cdcat}

The cdcat command changes the current working categories, and the command syntax is as follows:

cdcat [options] [target category name or path]

Options:

none: Change into the child category of the current working category.

$-u$ : Forces to search the target category name or path information in the whole system.

$-\mathrm{p}$ : Gives parent category options of the target category for users to choose one and work under it.

Example(s):

We use Figure 6.9 to show our example.

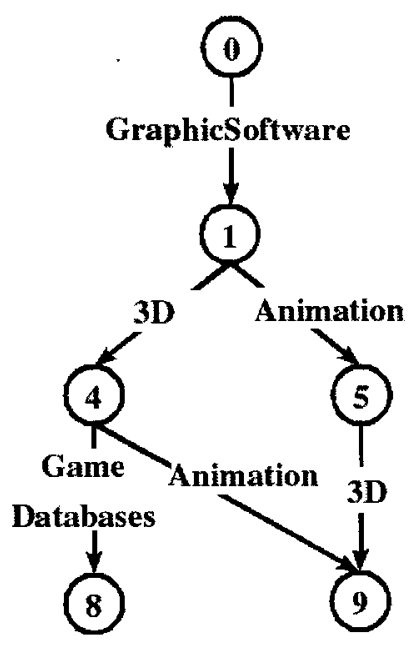

Figure 6.9: Example: cdcat 


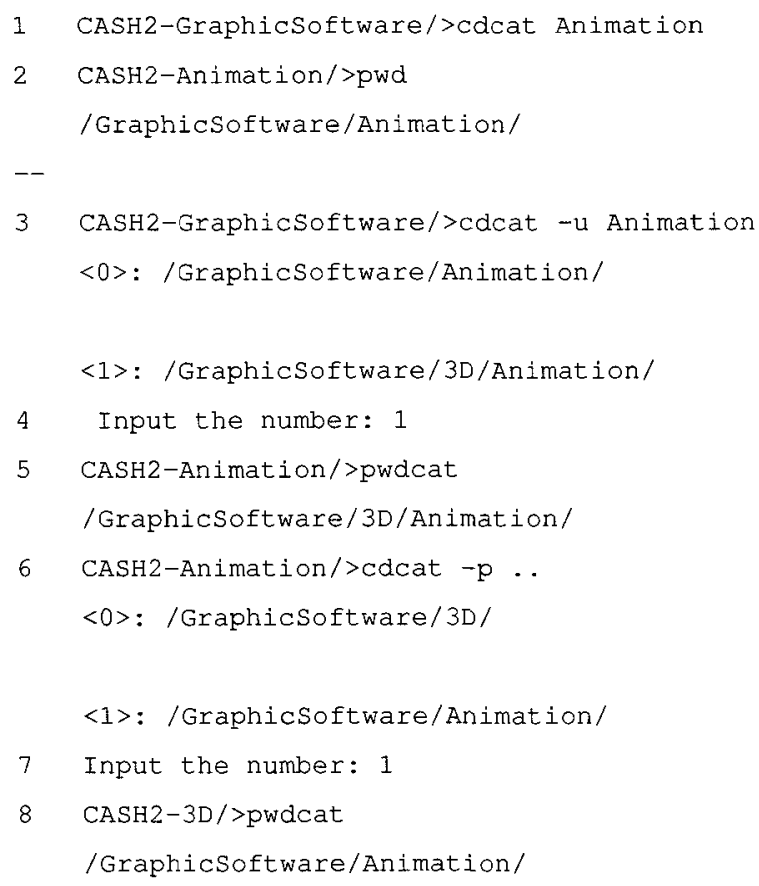

Explanation:

1. The category prompt shows that the current working category is $\mathbf{1}$ with the name "GraphicSoftware". The command cdcat Animation changes the working category to 1's child category 5 with the name "Animation".

2. The command pwdcat shows the current working category path. "/ GraphicSoftware/ Animation/"'.

3. In this line, the category prompt shows that the current working category name is "GraphicSoftware" again, however, if we use the command cdcat -u Animation, instead of changing the working category into the child category automatically, they system is forced to search for "Animation" in the whole category structure. The following two lines show two path information for "Animation".

4. In this line, we are asked to input our choice of which "Animation" we want to work with. Our choice is the second path. 
5. The command pwdcat shows the current working category path is changed to "/GraphicSoftware/3D/Animation/", which also means we are currently working under category 9.

6. The command cdcat $-\mathrm{p} \ldots$ is meant to change the working category into one of the parent categories of the input category. The following two lines show two parent category path options of the input category “.." (i.e., category 9).

7. We choose the second option, i.e., to work under category 5.

8. The command pwdcat shows the current working category path is changed to "/GraphicSoftware/ Animation/".

\subsubsection{Add Operations}

There are basically four add operations: add category, add category classification, add file, and add file classification operations. In $\mathrm{CASH} 2$, we use three commands to implement them, mkcat, classify and lncat.

\subsection{Command: mkcat}

This command is used to implement the add category operation, which creates a category entity in the Category table and establish the category classification relationship in the CategoryClassification table. The command syntax is as follows:

mkcat [options] [search path] [category name or path]

Options:

none: Creates new category under the current working category.

$-\mathrm{p}$ : Search path is presented, under which new category is created.

Example(s):

As shown in Figure 6.10, we would like to add three categories 14, 15 and 16 under the category 13, with their corresponding name "Champagne", "RedWine", and "WhiteWine". 


Calogory
\begin{tabular}{|c|c|c|c|c|c|c|}
\hline Cid & Cmode & Cotime & Catime & Cmtime & Cuid & Cgid \\
\hline 0 & 16877 & $2006-02-11$ & $2006-02-11$ & $2006-02-11$ & 1089 & 590 \\
\hline$\ldots$ & $\ldots$ & $\ldots$ & $\ldots$ & $\ldots$ & $\ldots$ & $\ldots$ \\
\hline 8 & 16877 & $2006-02-11$ & $2006-02-11$ & $2006-02-11$ & 1089 & 590 \\
\hline$\ldots$ & $\ldots$ & $\ldots$ & $\ldots$ & $\ldots$ & $\ldots$ & $\ldots$ \\
\hline 13 & 16877 & $2006-02-11$ & $2006-02-11$ & $2006-02-11$ & 1089 & 590 \\
\hline
\end{tabular}

Carlegory Classication
\begin{tabular}{|c|c|c|c|c|}
\hline ParentCid & Cid & Cname & Time & Cdescription \\
\hline 0 & 1 & Beverage & $2006-02-11$ & Null \\
\hline$\ldots$ & $\ldots$ & $\ldots$ & $\ldots$ & $\ldots$ \\
\hline 8 & 13 & Wine & $2006-02-11$ & Null \\
\hline
\end{tabular}

Before

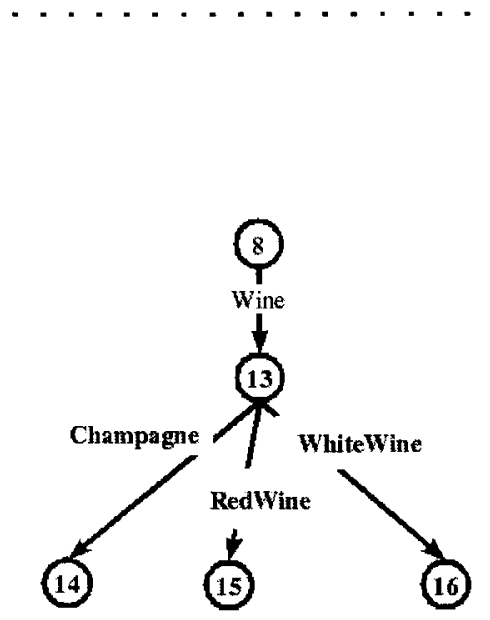

\begin{tabular}{|c|c|c|c|c|c|c|c|}
\hline \multicolumn{8}{|c|}{ Catcror } \\
\hline & Gid & Cmode & Cotime & Catime & Cmtime & Cuid & Cgid \\
\hline & 0 & 16877 & $2006-02-11$ & $2006-02-11$ & 2006-02-11 & 1089 & 590 \\
\hline & $\ldots$ & $\ldots$ & $\ldots$ & $\ldots$ & $\ldots$ & $\cdots$ & $\ldots$ \\
\hline Add & 13 & 16877 & $2006-02-11$ & 2006-02-11 & $2006-02-11$ & 1089 & 590 \\
\hline Add & 14 & 16877 & $2006-04-08$ & 2006-04-08 & $2006-04-08$ & 1089 & 590 \\
\hline Add & 15 & 16877 & 2006-04-08 & 2006-04-08 & $2006-04-08$ & 1089 & 590 \\
\hline Add & 16 & 16877 & 2006-04-08 & 2006-04-08 & 2006-04-08 & 1089 & 590 \\
\hline
\end{tabular}

\begin{tabular}{|c|c|c|}
\hline Cname & Time & Codescription \\
\hline Beverage & 2006-02-01 & Null \\
\hline$\ldots$ & & $\ldots$ \\
\hline Wine & $2006-04-01$ & Null \\
\hline Champagne & 2006-04-08 & Null \\
\hline RedWine & 2006-04-08 & Null \\
\hline WhiteWine & 2006-04-08 & Null \\
\hline
\end{tabular}

After

Figure 6.10: Add Category Operation 
1. If we are currently under the category $\mathbf{1 3}$ with the name "Wine", the command-line example is as follows:

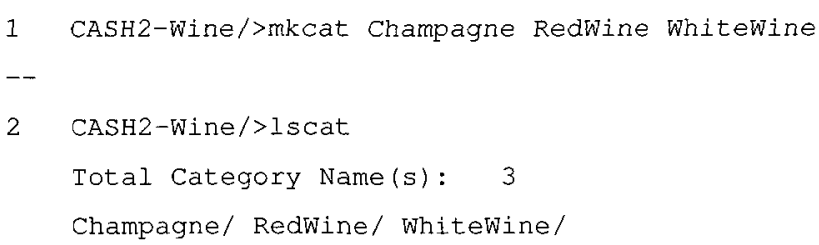

Explanation:

1. The command mkcat creates three categories with name "Champagne", "RedWine", and "WhiteWine" under the working category 13.

2. The command lscat shows the result.

2. If we are not currently under the category 13 , e.g., we are under the root category, we can use option $-\mathrm{p}$ to reach the category $\mathbf{1 3}$ and add new categories under it. Example(s):

1 CASH2-/>mkcat $-\mathrm{p}$ wine Champagne Redwine whitewine $--$

2 CASH2-1>lscat Wine

'Wine' does not exist under the current working category. Search? $y$ Champagne/ Redwine/ whitewine/

Explanation:

1. the system recognizes "Wine" as a category name or path that needs to be searched for, then locates category $\mathbf{1 3}$ and add the new categories under it.

2. The command lscat shows the result.

We describe the implementation in the following steps:

1. Add a record in table Category that contains the general information about the category. 
2. Add another record in table CategoryClassification, which represents the classification between the target category and an existing category.

\subsection{Command: classify}

This command is used to implement three operations: add category classification, add file, and add file classification operation. The following is the command syntax.

classify [options] [category name or path] [source]

[new category name or file logical name]

Options:

none: source is a category.

- $\mathrm{f}$ : source is a file or directory in directory format.

-c: source is a logical file name in category format.

We now explain the command with regards to the operation it implements.

1. Add Category Classification Operation

This operation is used to establish a category classification relationship between two categories in the CategoryClassification table. The command format is as the following:

classify [parent category name or path]

[child category name or path]

[new category name]

Example(s):

As shown in Figure 6.11, we would like to classify category $\mathbf{1 1}$ to $\mathbf{3}$ with the name "Cola", i.e., to build a parent-child relationship between $\mathbf{1 1}$ and $\mathbf{3}$. We are now under the root category, and the command-line example is as follows:

1 CASH2-/>classify/Beverage/Carbonated ।

/Beverage/NonAlcoholic/Caffeinated/Cola 


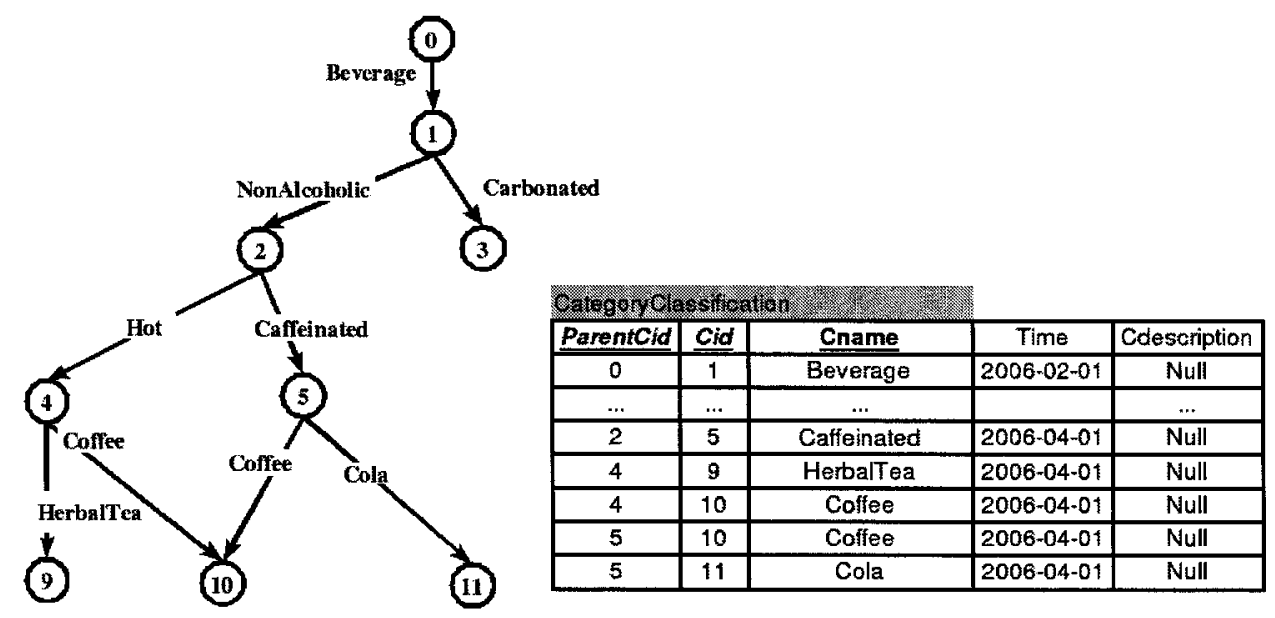

Before ....................

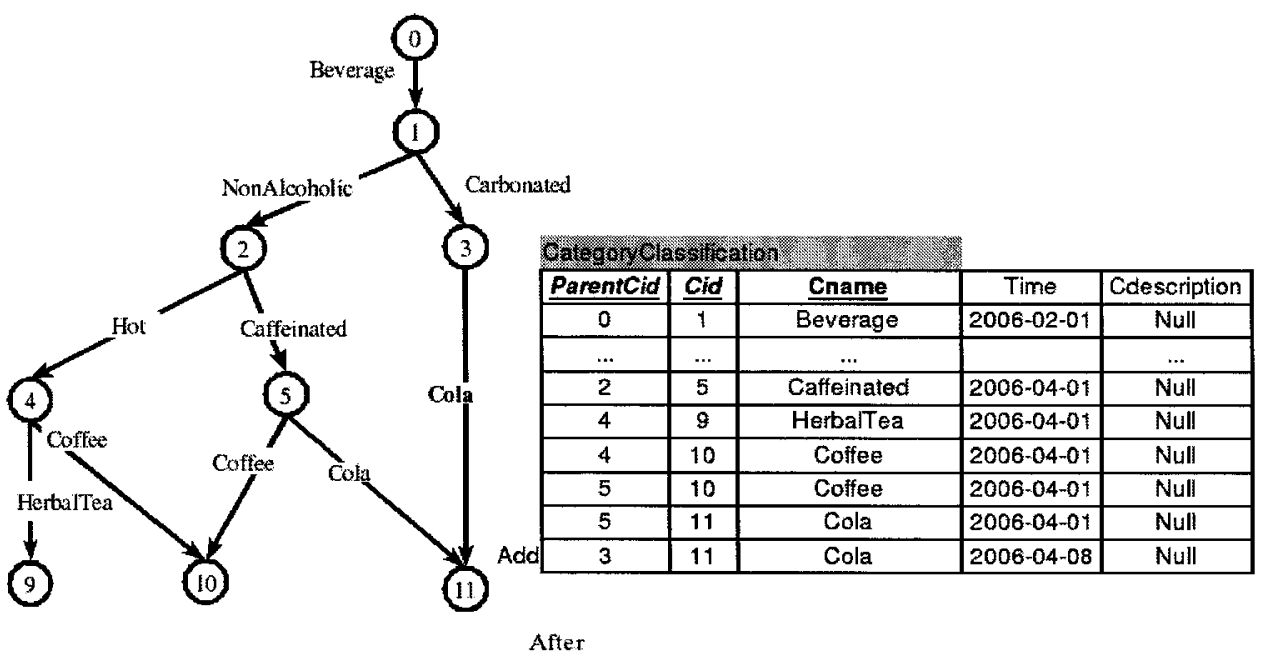

Figure 6.11: Add Category Classification Operation 
2 CASH2-1>lscat Carbonated

'Carbonated' does not exist. Search? y

Total Category Name(s): 1

Cola/

Explanation:

1. In this line, "/ Beverage/ Carbonated" is the parent category path information, and

“/ Beverage/ NonAlcoholic/ Caffeinated/ Cola" is the child category path information. Because no new category name is provided, by default, the system uses the last category name "Cola" in the child category path as the new category name.

2. Is cat shows the result that "Cola" is classified under "Carbonated".

After the operation, a new record that builds up the relationship between the category $\mathbf{1 1}$ and $\mathbf{3}$ is added in table CategoryClassification.

2. Add File Operation This operation is used to create a file entity in the File table, create a related directory entity in the Directory table (if necessary), and establish the file classification relationship in the FileClassification table. The command format is as the following:

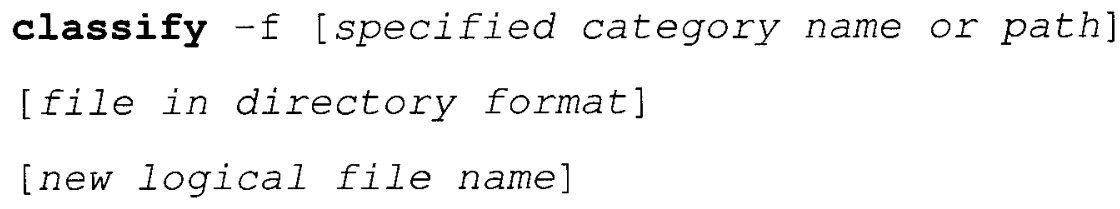

Example(s):

As shown in Figure 6.12, we would like to classify the file 8 to category 14 (File id $\mathbf{8}$ is used to simplify the graph representation. The actual id is the primary key value in table File). The command-line example is as follows:

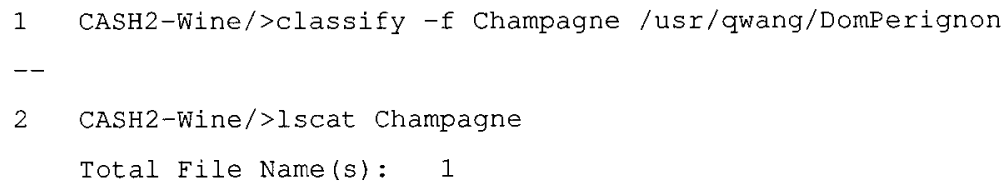


Explanation:

1. "/ usr/ qwang/ DomPerignon" is the file "DomPerignon" in a directory path form. "Champagne" is the category name we would like to classify the file under. Since no new logical file name is provided, by default, the system uses the physical file name "DomPerignon" as the new logical file name.

2. The command lscat shows the result.
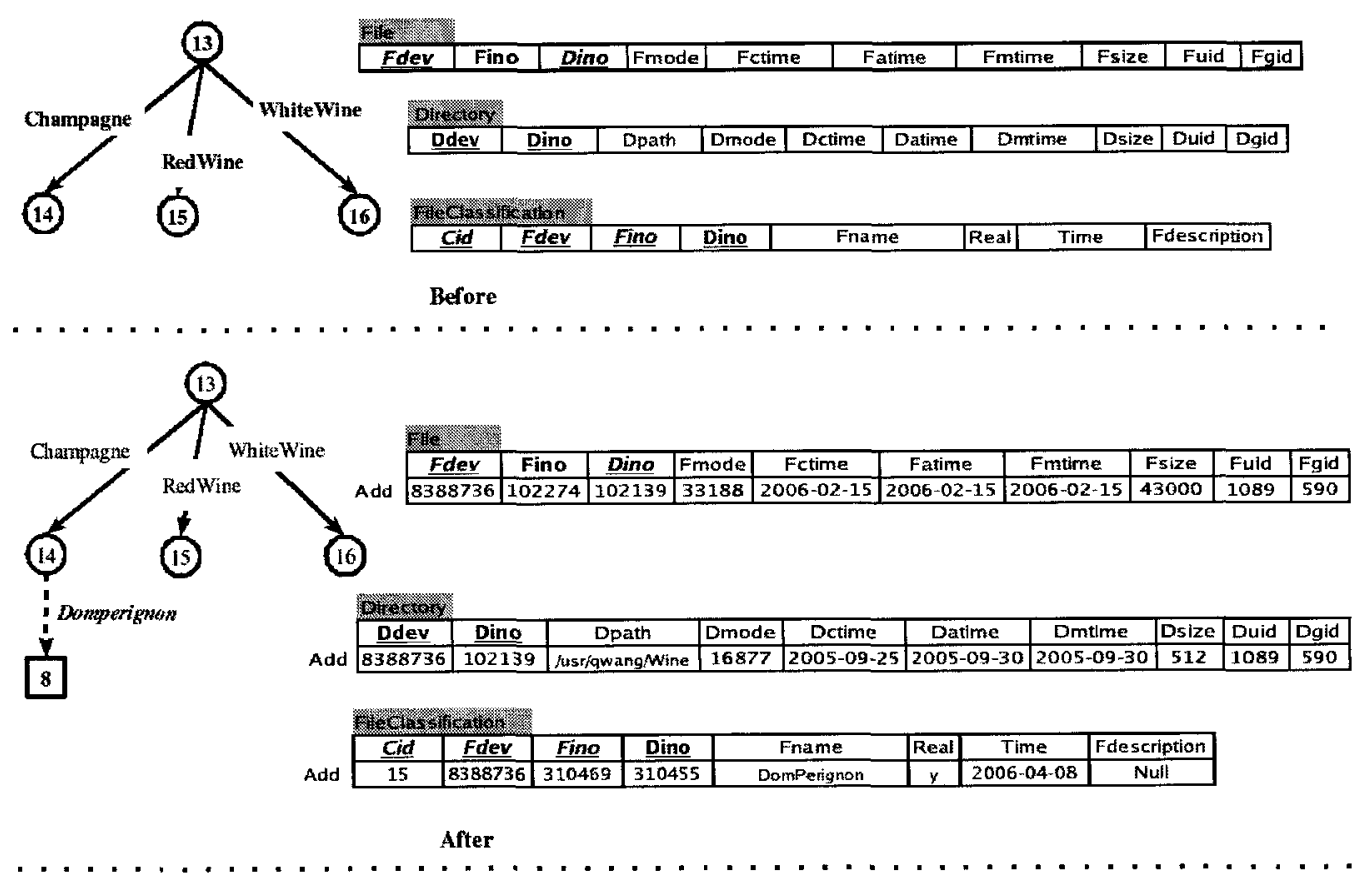

Figure 6.12: Add File Operation

We describe the implementation in the following steps:

(a) Add a record in table File that contains the general information about the target file.

(b) Add a record in table Directory that contains the general information about the directory the target file is located in, if the record already exists, skip this step. 
(c) Add another record in table FileClassification, which represents the classification between the target file and the category it classified to.

3. Add File Classification Operation This operation is used to build up a relationship between an existing file entity and a category in the FileClassification table. We use option $-\mathrm{c}$ to implement this operation. The command format is as the following:

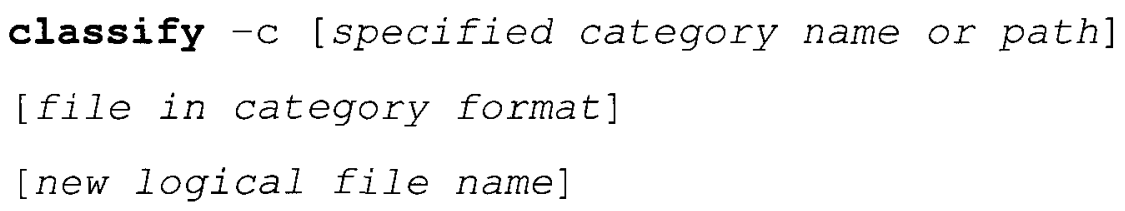

Example(s):

As shown in Figure 6.13, we would like to classify file 5 to category 3 with logical file name "PerrierMineralWater". Because file $\mathbf{5}$ is already classified to the category 2, and exists in our system, the operation only needs to add one more record in the table FileClassification. The command-line example is as follows:

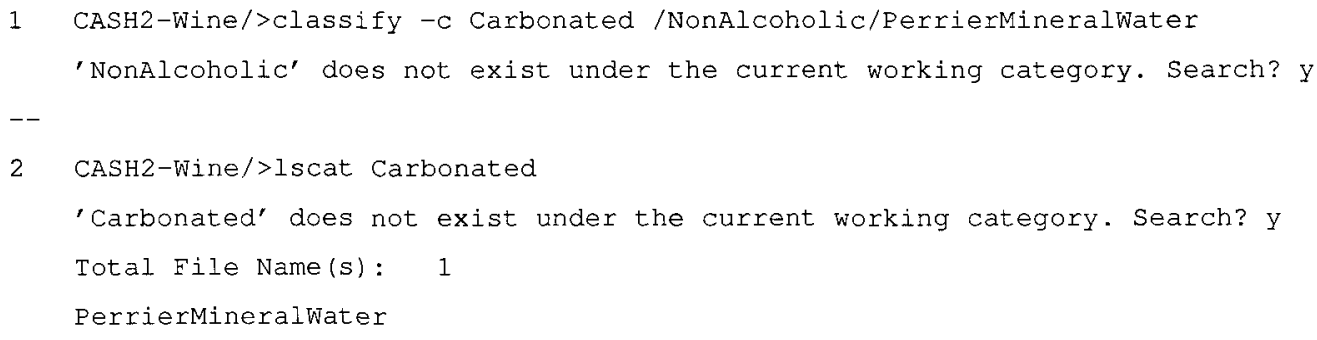

Explanation:

1. In this line, "/ NonAlcoholic/ PerrierMineralWater" is a category format for the target file "PerrierMineralWater", and "NonAlcoholic" is the category path information. "Carbonated" is the specified category path information, under which, we would like to classify the target file. Because no new logical file name is provide, by default, the system uses "PerrierMineralWater" obtained from the path information of the target file, as the new logical file name.

2. Iscat display the result. 
After the operation, one new record that builds up the relationship between the category $\mathbf{3}$ and the file $\mathbf{5}$ is added into table FileClassification.
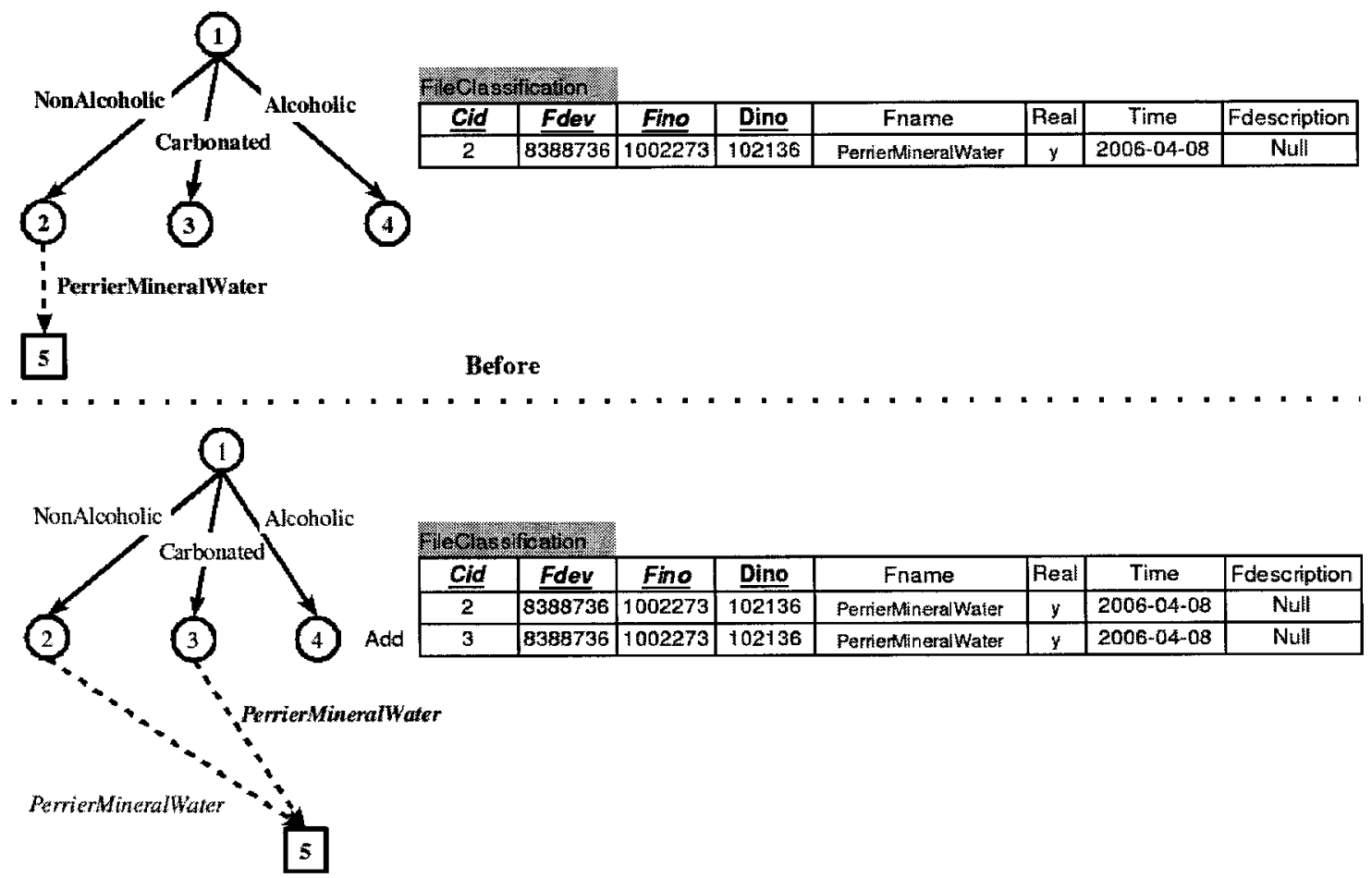

After

Figure 6.13: Add File Classification Operation

\subsection{Command: Incat}

This command is used to create alias for a category name. Beside the command classify, Incat also implements the add category classification operation. The command syntax is as follows:

lncat [ Target]

or

lncat [Target] [alias name]

Example(s): 
Figure 6.11 demonstrates this operation in two cases.

1 CASH2-Carbonated/>lncat /Beverage/NonAlcoholic/Caffeinated/Cola

$--$

2 CASH2-Carbonated/>Incat /Beverage/NonAlcoholic/Caffeinated/Cola myCola

Explanation:

1. This line shows that working category name is "Carbonated", and this command line is meant to create an alias category name "Cola" under the current working category, point to "/ Beverage/ NonAlcoholic/ Caffeinated/ Cola". The example is shown in Figure 6.14 (a).

2. This command line is meant to create an alias category name "myCola" under the current working category, point to "/ Beverage/ NonAlcoholic/ Caffeinated/Cola". The example is shown in Figure $6.14(\mathrm{~b})$.

After the operation, one new record that builds up the relationship between the category $\mathbf{1 1}$ and $\mathbf{3}$ is added into table CategoryClassification.

\subsubsection{Remove Operation}

There are four remove operations, remove file, remove file classification, remove category and remove category classification operations. In CASH2, we use two commands to implement them, rmcat and decat.

\subsection{Command: rmcat}

This command is used to implement two operations: remove files and remove categories. Both operations may involve physical files to be removed from the disk. the command syntax is as follows:

rmcat [option] [Target]

Options:

none: target is a category name or path.

$-\mathrm{f}$ : target is a logical file name in category format, and all the categories in the sub-DAG 

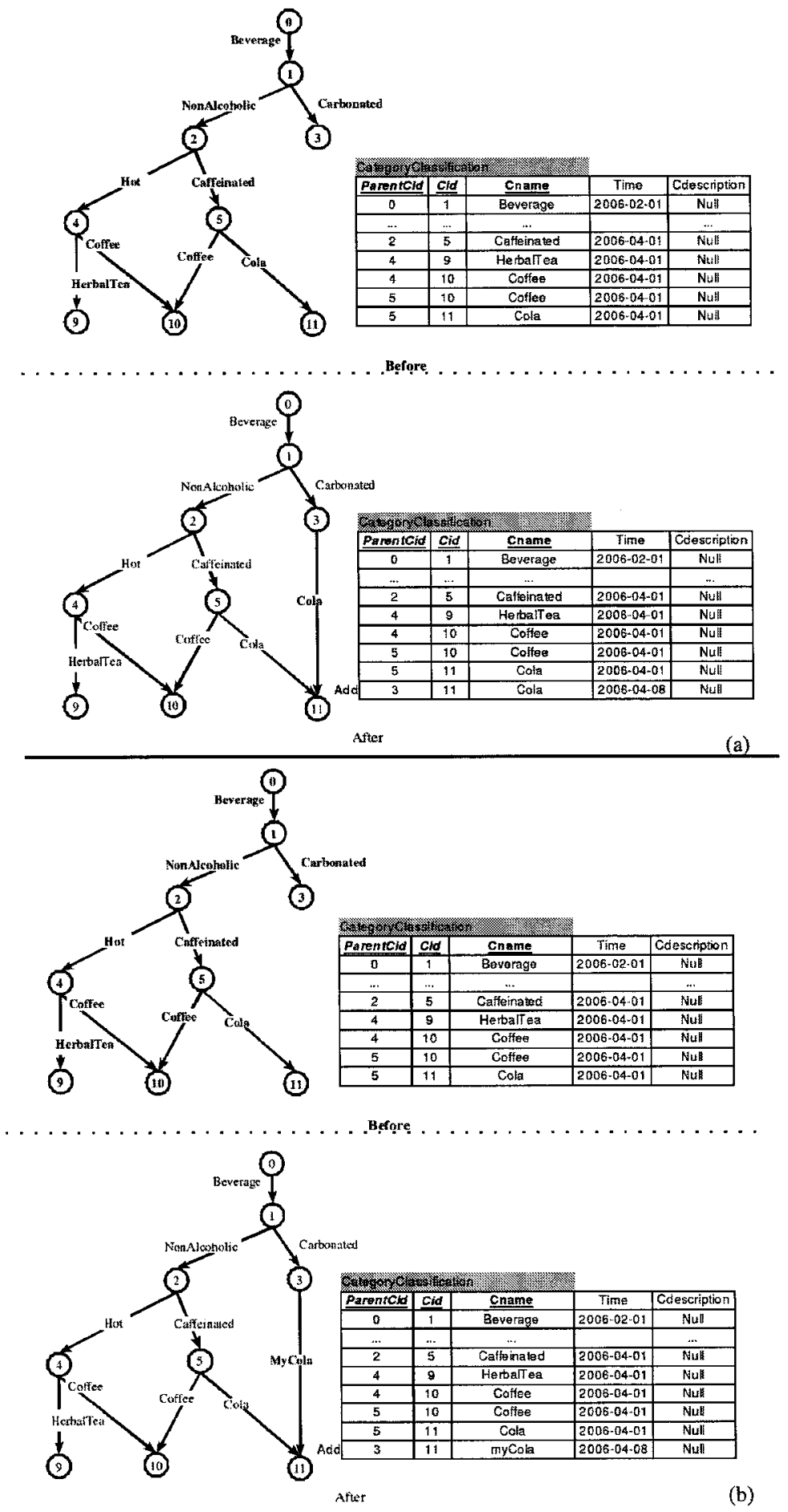

Figure 6.14: Example: Incat 
rooted at target will be removed.

$-\mathrm{k}$ : target is a category name or path, and all the categories in the sub-DAG rooted at target that do not have aliases that do not belong to the sub-DAG will be removed.

-a: Clear all the data in the system.

We now explain the command with regard to the operation it implements.

1. Remove File Operation

This operation is used to remove a target file entity from the File table, remove the file physically from the disk, remove the associated file classification relationships in the FileClassification table, and a related directory entity from the Directory table if necessary. The command format is as follows:

rmcat $-f$ [logical file name in category format]

Example(s):

As shown in Figure 6.15, we would like to remove the file $\mathbf{1 0}$ from our system. The command-line example is as follows:

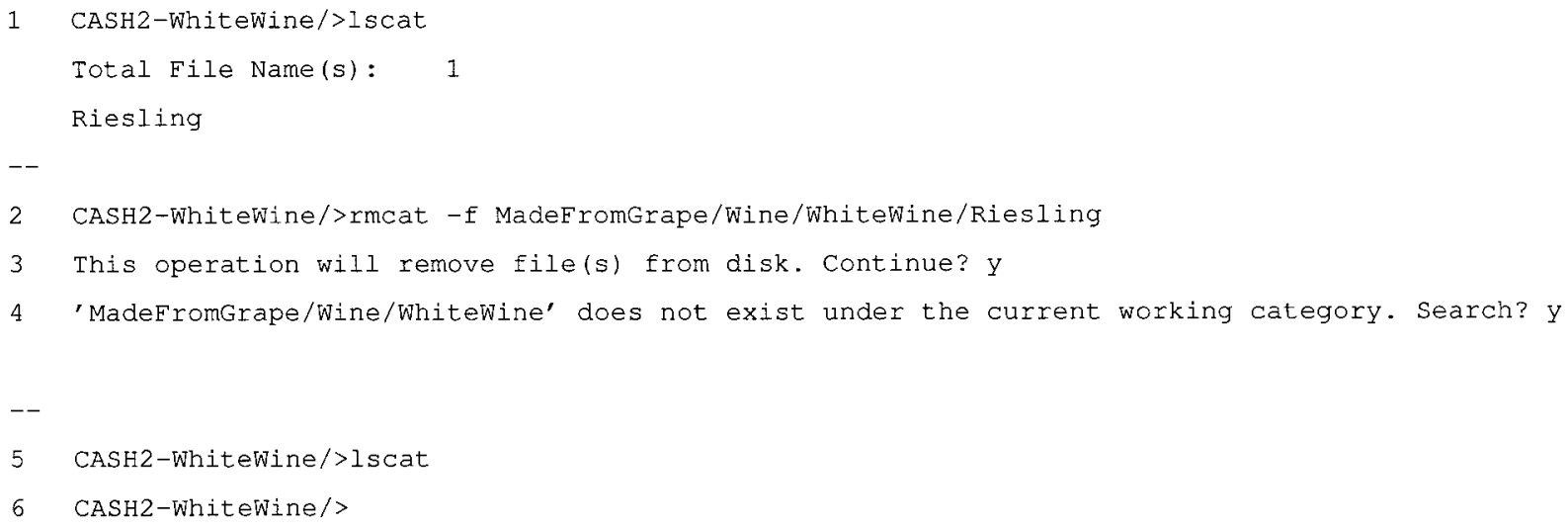

\section{Explanation:}

1. The command lscat shows that file "Riesling" is classified under "WhiteWine".

2. The file "Riesling" is in category format as it is under a category path "MadeFromGrape/ Wine". 

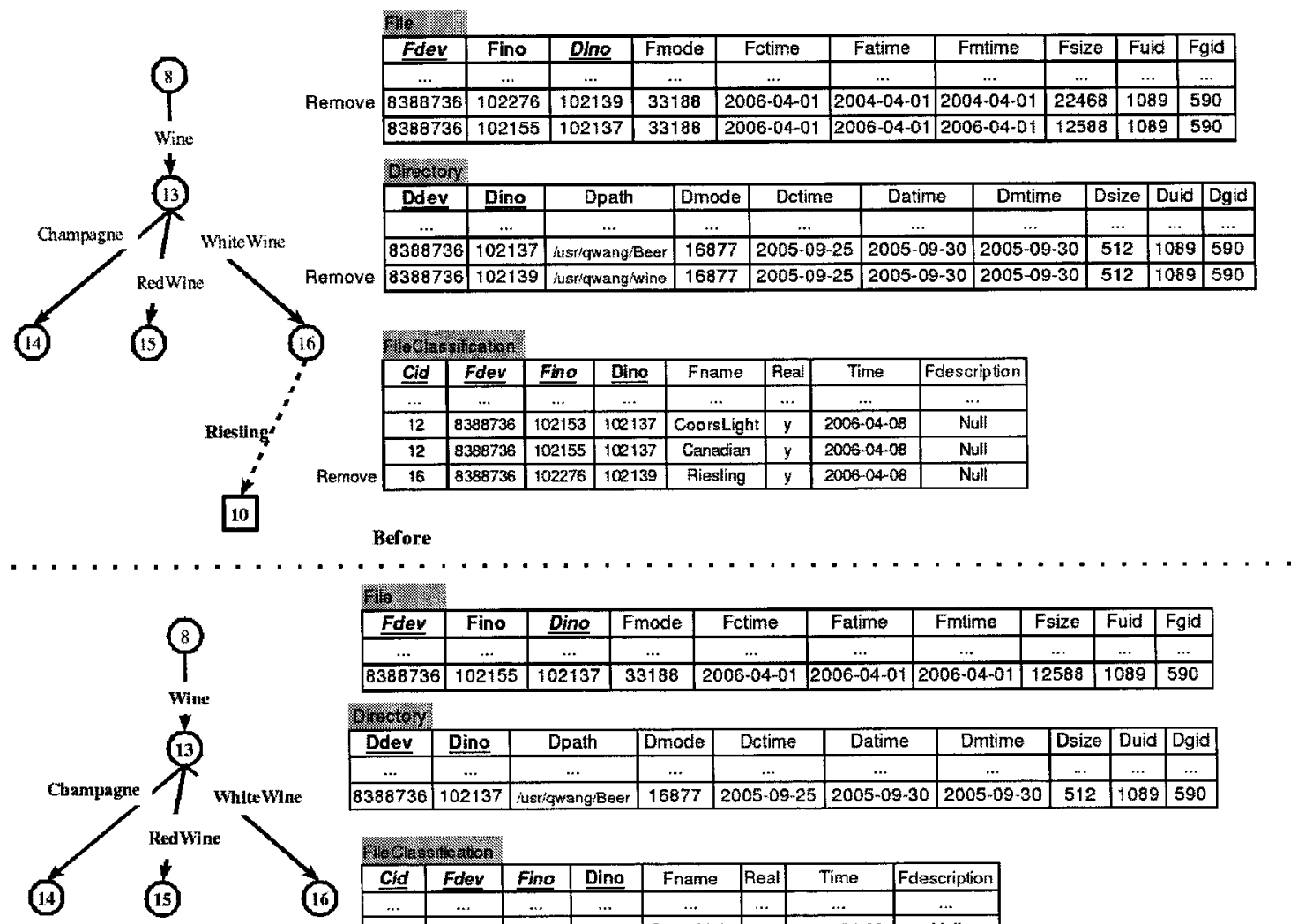

(m)

\begin{tabular}{|c|c|c|c|c|c|c|c|c|c|}
\hline Fdey & Fino & Dino & Fmode & Fctime & Fatime & Fmtime & Fsize & Fuid & Fgid \\
\hline$\ldots$ & $\ldots$ & $\ldots$ & $\ldots$ & $\ldots$ & $\ldots$ & $\ldots$ & $\ldots$ & $\ldots$ & $\ldots$ \\
\hline $\mathbf{B 3 8 8 7 3 6}$ & 102155 & 102137 & 33188 & $2006-04-01$ & $2006-04-01$ & $2006-04-01$ & 12588 & 1089 & 590 \\
\hline
\end{tabular}

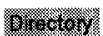

\begin{tabular}{|c|c|c|c|c|c|c|c|c|c|}
\hline Ddev & Dine & Dpath & Dmode & Dctime & Datime & Dmtime & Dsize & Duid & Dgid \\
\hline$\ldots$ & $\ldots$ & $\ldots$ & $\ldots$ & $\ldots$ & $\ldots$ & $\ldots$ & $\ldots$ & $\ldots$ & $\ldots$ \\
\hline 8388736 & 102137 & $\ldots$ & $\ldots$ & $\ldots$ & $\ldots$ & & $\ldots$ \\
\hline
\end{tabular}

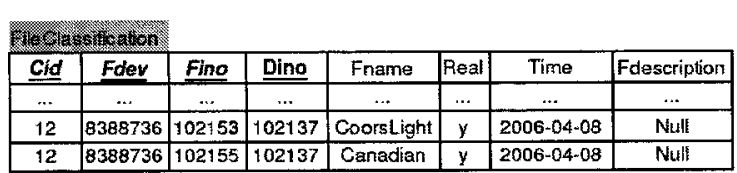

After

Figure 6.15: Remove File Operation 
3. The system gives a warning that this remove operation will remove file(s) physically.

4. As the current working category name is "Wine", and path "MadeFromGrape/ Wine" is not under it, the system asks if we want to search for it. After we confirm "yes", the file is removed from CASH2 and disk as well.

5. The command lscat shows the result. There is no file currently classified under "WhiteWine".

The implementation is described in the following steps:

(a) Remove the target file record in table File, and remove the physical file from the disk as well.

(b) Remove the directory record in table Directory which contains the directory information the target file is located in (skip this step if the table File still contains files that under this directory).

(c) Remove the record in table FileClassification, which represents the classification between the file and the target category it is classified to.

\section{Remove Category Operation}

This operation is used to remove the target category entity from table Category, remove all its classifications in the parent categories and all the descendants of the target category and their classification in their parent categories (if there is any) from table CategoryClassification, remove all the files classified to the target category and its descendants in table File (if necessary), and remove all the related directories in table Directory (if necessary). The command format is as follows:

rmcat [category name or path]

Example(s):

As shown in Figure 6.16, we would like to remove the category 13. The working category is the root, and the command-line example is as follows: 


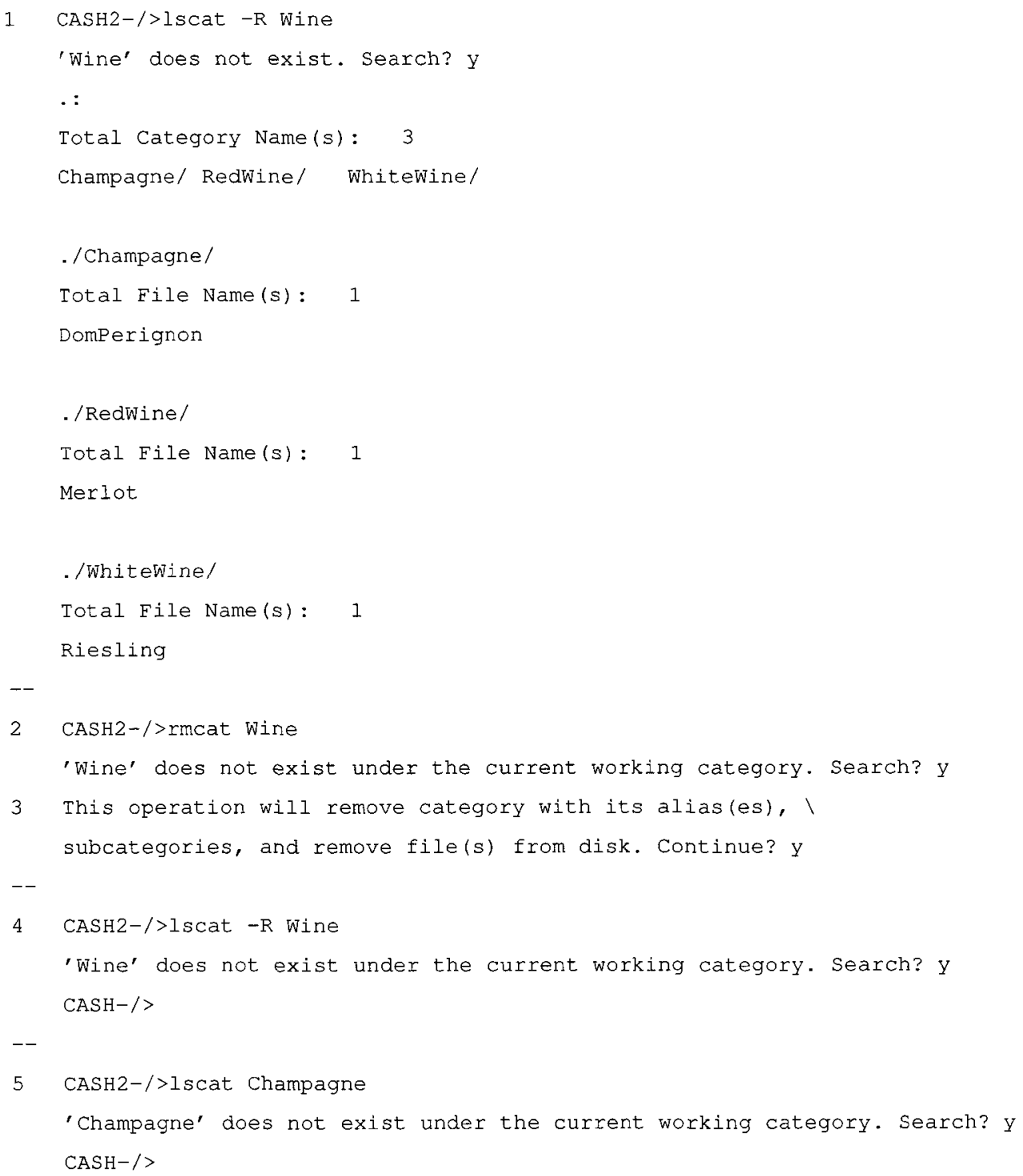

\section{Explanation:}

1. The command lscat -R shows that under the category name "Wine", there are there category "Champagne", "RedWine", and "WhiteWine", and each of them has a file under it. The file names are "DomPerignon", "Merlot", and "Riesling" accordingly.

2. rmcat Wine is meant to remove the category $\mathbf{1 3}$ with the name "Wine" from 


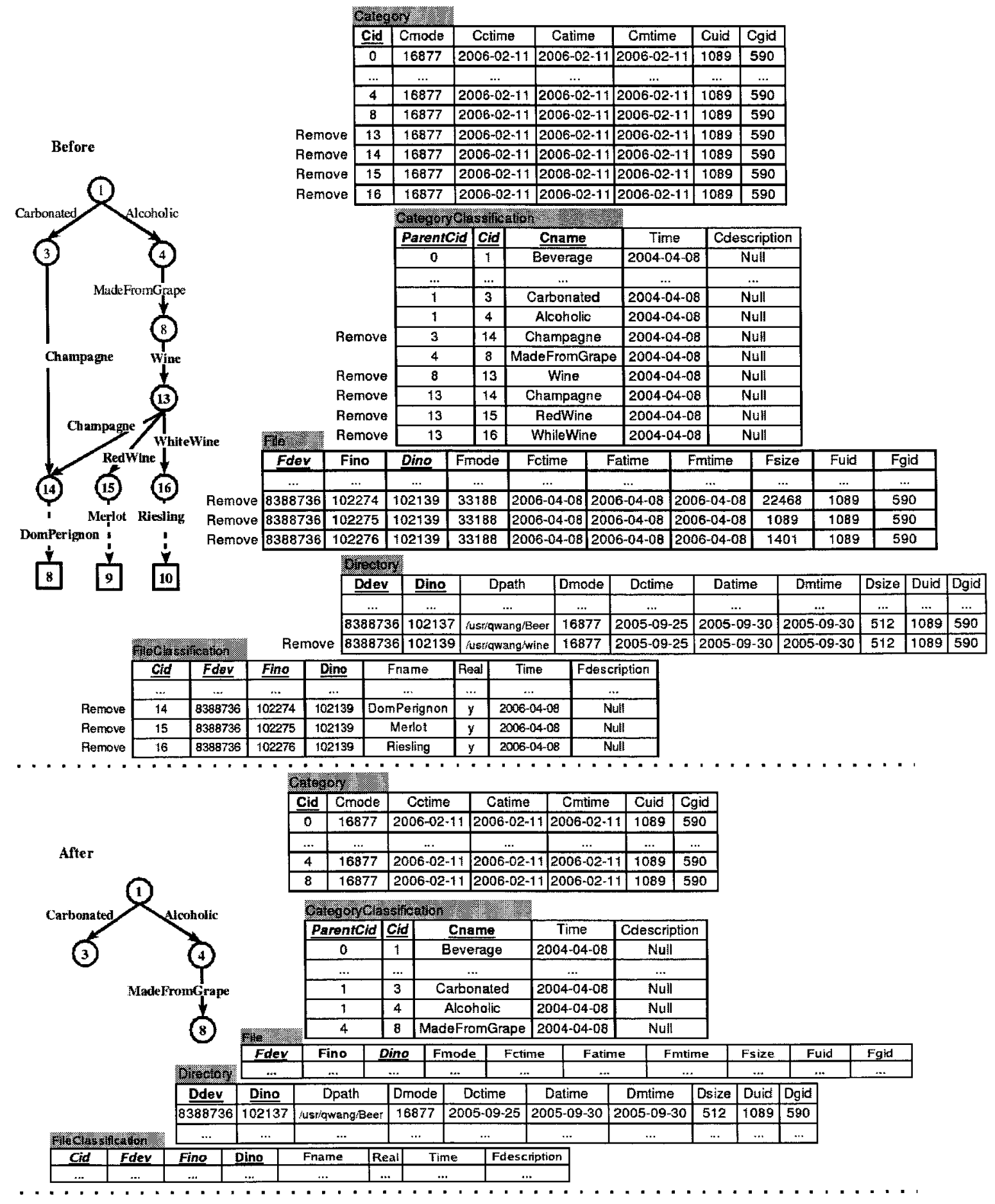

Figure 6.16: Remove Category Operation 
the system.

3. After locating the category $\mathbf{1 3}$ with the name "WhiteWine", they system recognizes the target category as an inner node, which means it may have alias(es) and descendant categories with files classified to them. Then, it gives a warning informing users that all of the related categories and files classified to them will be removed from CASH2 and from the disk. The system will then let users choose whether they want the operation to continue. After we confirm yes, all the information above is removed.

4. Iscat $-\mathrm{R}$ Wine shows that category "Wine", and all its sub-categories are removed.

5. Iscat Champagne shows that there is no "Champagne" in the system any more.

The implementation is described in the following steps:

(a) Remove the category record in the table Category.

(b) Perform the remove file operation for all the files classified to the target category.

(c) Remove all the classifications of the category in the parent category(s) by deleting the corresponding record(s) in the table CategoryClassification.

(d) Remove all the descendants of the target category by deleting all the corresponding category records in the table Category (Skip this step if the category does not have any descendant).

(e) Perform the remove file operations for all the files classified to all the descendant categories (Skip this step if the category does not have any descendant).

(f) Remove all the classifications of each descendant by deleting the corresponding record(s) in the table CategoryClassification (Skip this step if the category does not have any descendant). 
The command rmcat also have another option $-k$ to remove categories, the difference between the $-\mathrm{k}$ option and the operation we mentioned above is that, rmcat $-\mathrm{k}$ keeps some categories without being removed, under the condition that these categories have aliases that do not belong to the sub-DAG rooted at the input category. Example(s):

As shown in Figure 6.17, we would like to remove the category 13. The working category is the root, and the command-line example is as follows:

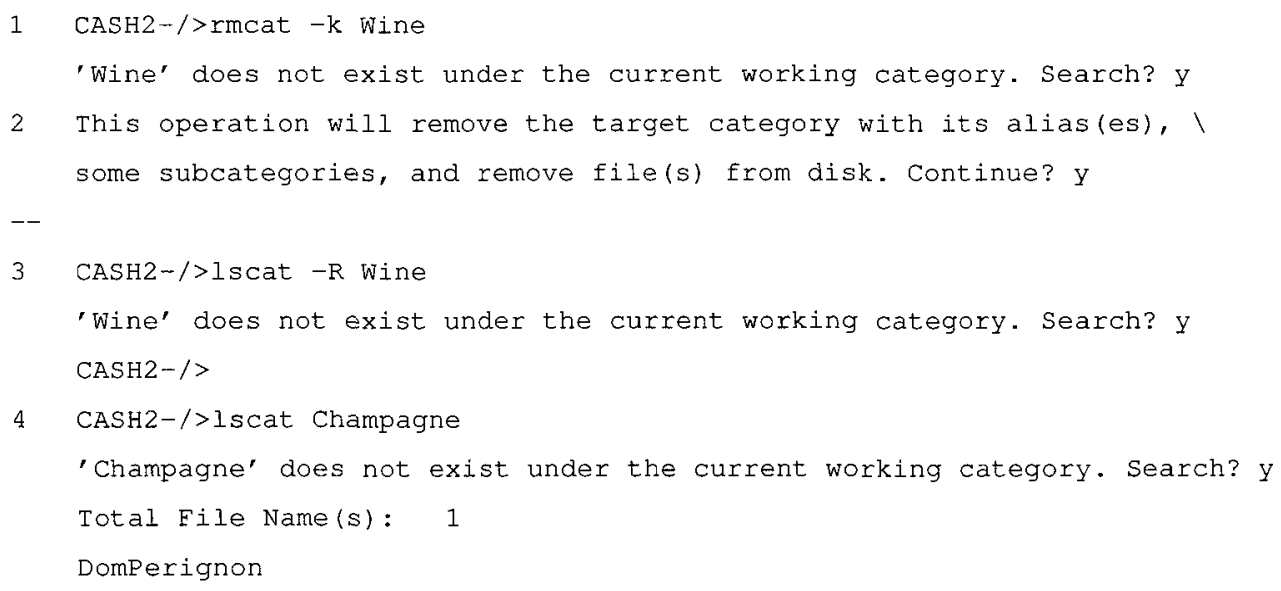

Explanation:

1. rmcat $-\mathrm{k}$ Wine is meant to remove the category $\mathbf{1 3}$ with the name "Wine" and all its sub-categories without aliases from the system.

2. After locating the category $\mathbf{1 3}$ with the name "WhiteWine", they system recognizes the target category as an inner node, which means it may have alias(es) and descendant categories with files classified to them. Then, it gives a warning informing users that all of the related categories and files classified to them will be removed from CASH2 and from the disk. The system will then let users choose whether they want the operation to continue. After we confirm yes, category 15 and $\mathbf{1 6}$ is removed. 3. Iscat $-\mathrm{R}$ wine shows that category $\mathbf{1 3}, \mathbf{1 5}$ and $\mathbf{1 6}$ are removed. 


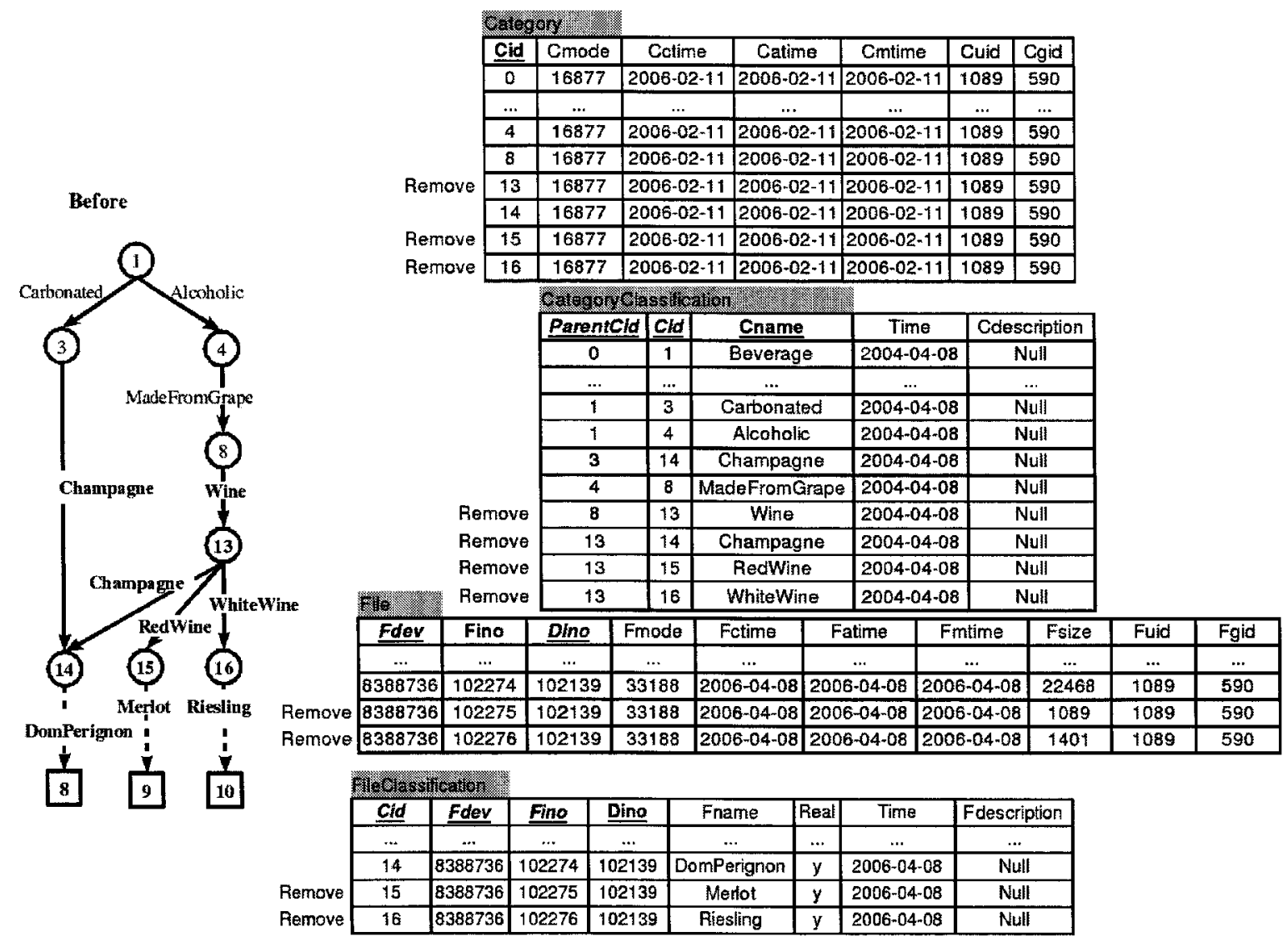

After

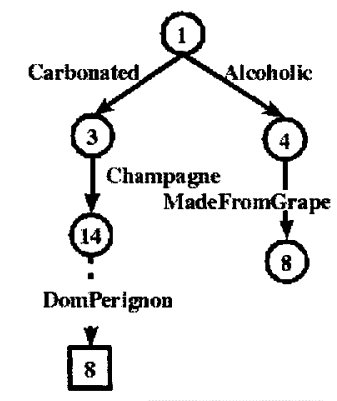

\begin{tabular}{|c|c|c|c|c|c|c|}
\hline Cid & Cmode & Cotime & Catine & Contime & Cuid & Cgid \\
\hline 0 & 16877 & 2006-02-11 & $2006-02-11$ & 2006-02-11 & 1089 & 590 \\
\hline$\ldots$ & $\ldots$ & $\ldots$ & $\ldots$ & $\ldots$ & $\ldots$ & $\ldots$ \\
\hline 4 & 16877 & $2006-02-11$ & $2006 \cdot 02-11$ & 2006-02-11 & 1089 & 590 \\
\hline 8 & 16877 & 2006-02-11 & 2006-02-11 & 2006-02-11 & 1089 & 590 \\
\hline 14 & 16877 & $2006 \cdot 02-11$ & 2006-02-11 & $2006-02-11$ & 1089 & 590 \\
\hline
\end{tabular}

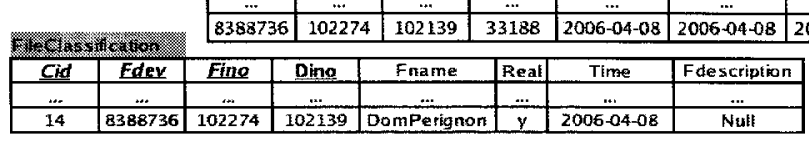

Figure 6.17: Example of rmcat $-k$ 
4. Iscat Champagne shows that category $\mathbf{1 4}$ and the file "DomPerignon" still exist.

\subsection{Command: decat}

This command is used to implement two operations: remove file classification and remove category classification. Compared to the command rmcat, decat removes categories and files neither from the system nor from the disk. The command syntax is as follows:

decat [option]

[Category name or path]

[ Target]

Options:

none: target is a category name.

- $\mathrm{f}$ : target is a logical file name.

We now explain the command with regard to the operation it implements.

1. Remove File Classification Operation

This operation is used to remove a file classification in its specified category from table FileClassification. The command format is as follows:

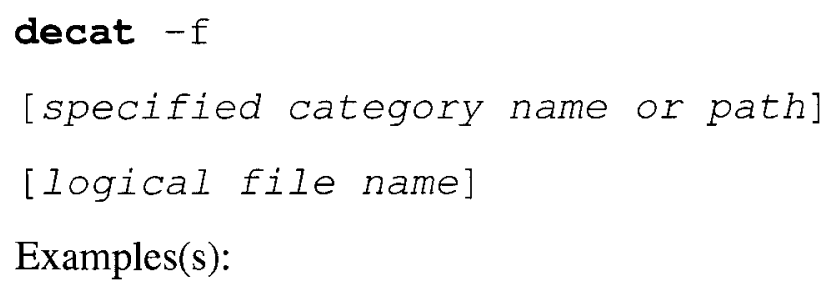

As shown in Figure 6.18, we would like to remove the file logical name "PerrierMineralWater" from the category 2. The working category is the root, and the command-line example is as follows:

1 CASH2-/>decat -f NonAlcoholic PerrierMineralwater

'NonAlcoholic' does not exist under the current working category. Search? y 


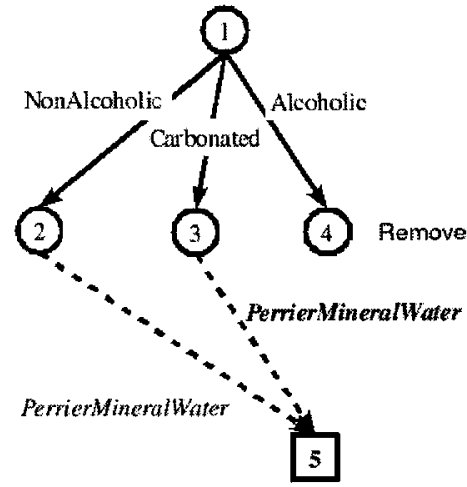

Ciectassifestien
\begin{tabular}{|c|c|c|c|c|c|c|c|}
\hline Cid & Fdey & Fino & Dino & Fname & Real & Time & Fdescription \\
\hline 2 & 8388736 & 1002273 & 102136 & PernerMineralWater & $y$ & $2006-04-08$ & Null \\
\hline 3 & 8388736 & 1002273 & 102136 & PernerMineralWater & $y$ & $2006-04-08$ & Null \\
\hline
\end{tabular}

Before

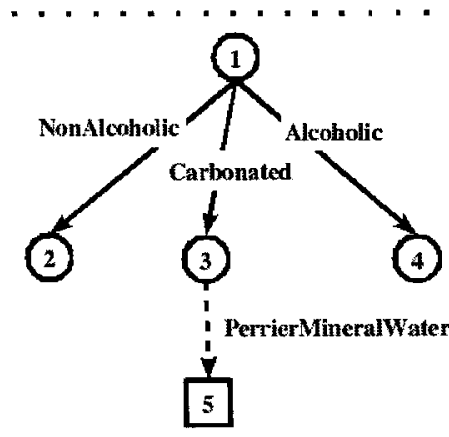

\section{(1) 8 .}

\begin{tabular}{l|l|l|l|l|l|l|l|}
\hline Cid & Fdev & Fino & Dino & Fname & Real & Time & Fdescription \\
\hline
\end{tabular}

\begin{tabular}{c|c|c|c|c|c|c|c|} 
Cid & Fdev & Fino & Dino & Fname & Real & Time & Fcescript \\
\hline 3 & 8388736 & 1002273 & 102136 & PerrierMineralWater & y & 2006-04-08 & Null
\end{tabular}

4.)

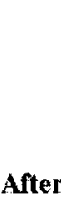

Figure 6.18: Remove File Classification Operation 
2 CASH2 $-/>$ lscat $-F$ Per*

/Beverage/Carbonated/PerrierMineralwater

Explanation:

1. decat $-\mathrm{f}$ is meant to remove the logical file name, "PerrierMineralWater" from its parent category with the name "NonAlcoholic".

2. After the removing operation, $l s c a t$ shows the result that "PerrierMineralWater" has only one category path associated with it. After the operation, the record in table FileClassification that represents the classification between file $\mathbf{5}$ and category $\mathbf{2}$ is removed.

2. Remove Category Classification Operation

This operation is used to remove a category classification in its parent category from table CategoryClassification. The command format is as follows:

decat

[parent category name or path]

[child category name]

Examples(s):

As shown in Figure 6.19, we would like to remove the category 11's classification in 3. The working category is the root, and the command-line example is as follows:

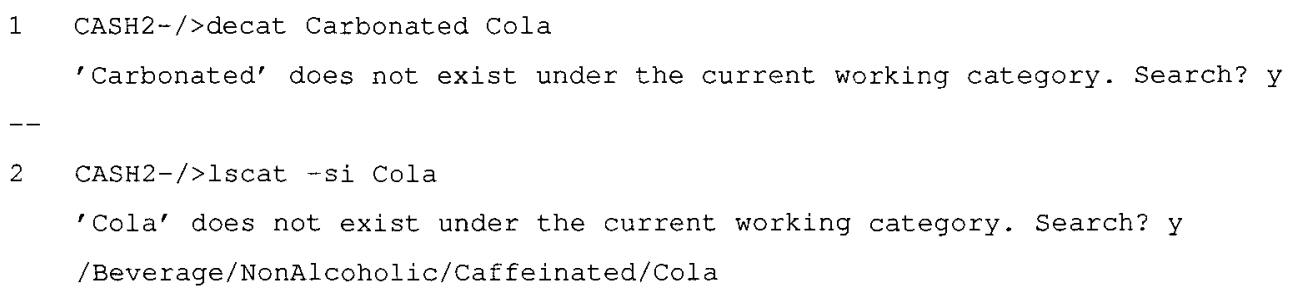

Explanation:

1. In this line, "Cola" is the child category name, and "Carbonated" is the parent category name. Since "Carbonated" is not under the root category, the system asks if we 

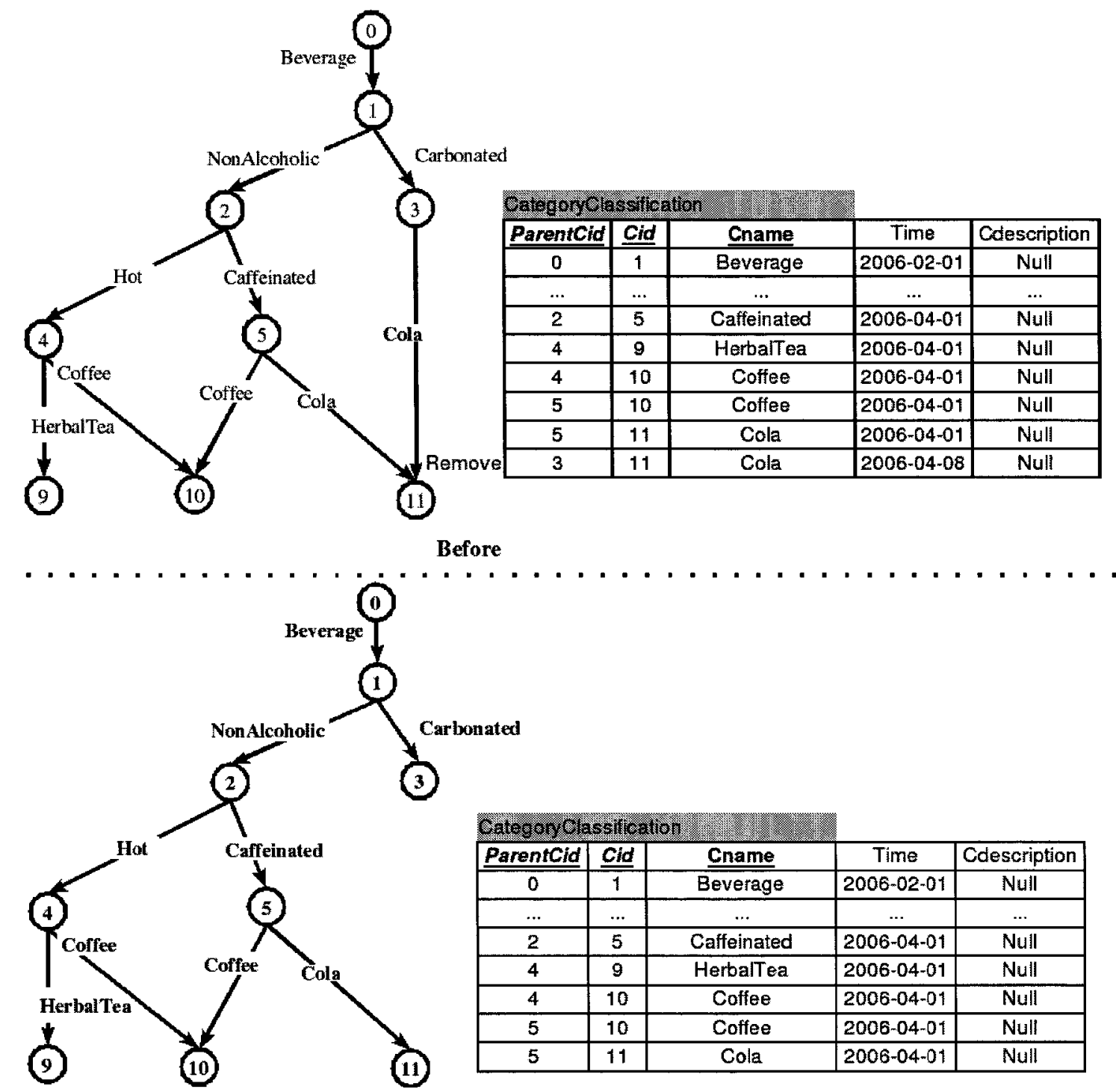

After

Figure 6.19: Remove Category Classification Operation 
want to search for it. After we confirm "yes", the system locates it and de-classifies its child category name "Cola" from it.

2. After the removing operation, lscat -si shows all the path information of "Cola". the result the only one path information left in the system, "/ Beverage/ NonAlcoholic/ Caffeinated/ Cola". After the operation, the record in table CategoryClassification that represents the classification between category $\mathbf{1 1}$ and category $\mathbf{3}$ is removed.

\subsubsection{Other Operations}

In this section, we explain some other operations implemented by the command category and directory, load, and pwdcat.

\subsubsection{Command: category and directory}

CASH2 provides two types of prompt displaying: Category Prompt and Directory Prompt. The default is the directory prompt. The command category switches the default prompt to the category prompt, and the command directory switches it back. The category prompt is formatted as follows:

CASH2 [current working category name]>

Example(s):

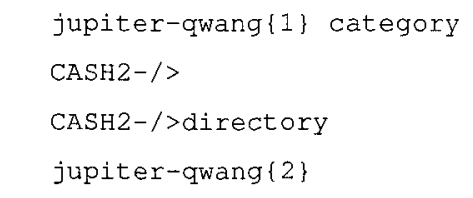

Explanation:

1. The command category switches the current directory prompt to the category prompt.

2. The category prompt shows that the current working category is the root category . 
3. The command directory switches the current category prompt to the directory prompt.

\subsubsection{Command: load}

The load command maps the existing directory hierarchy into CASH2. All the directories are mapped as pre-defined categories. This allows a file to automatically inherit this classification in the directory structure. The links are mapped as aliases, and the command syntax is as follows:

load [directory]

For example, the following command maps the root directory and all its sub-directories into CASH2.

CASH2-/>10ad /

\subsubsection{Command: pwdcat}

The pwdcat command shows the current working category path.

The following example shows that the current working category path is "/ Beverage/ NonAlcoholic/ Hot".

CASH2-Hot/>pwdcat

/Beverage/NonAlcoholic/Hot/

\subsection{Berkeley DB Implementations}

\subsubsection{Using C Structures with Pointers for Database Record}

In BDB, a database is a collection of records. They contains two parts, a key and some data. Both the key and its corresponding data are encapsulated in DBT structures, which provide a void * field that we can use to point to our data, and another field that identifies the 
data length. It is necessary to store data in structures to pack varied types of information into each database record. A C structure that contains fields without pointers is stored in the database with a fixed size. This, however, does not save any space from storing i.e. a category name with 2 characters versus a category name with 20 characters. The wasted space in each record will make the database larger than it should be, and require a larger cache and more disk $I / O$ than needed [41]. As a result, we choose to use fields with pointers in our $\mathrm{C}$ structures to create a variable size data. Figure 6.20 shows the $\mathrm{C}$ structures of records in all the tables. To store the structures, we pack the data into a single memory, and then store the data in that location. To retrieve the data, we use the data length that stored in the DBT structures to get the same number of bytes into the memory, then we unpack the data back into the $\mathrm{C}$ structures we defined.

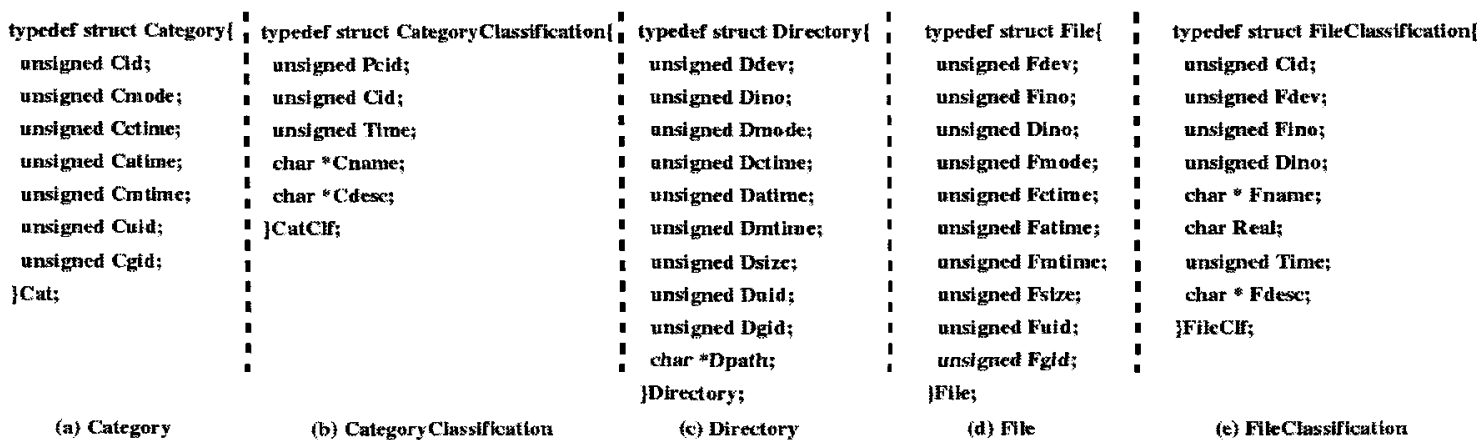

Figure 6.20: C Structures of Records in CASH2 Tables

\subsubsection{Primary and Secondary Databases}

In $\mathrm{BDB}$, the database that contains all the data is called a primary database. A database that provides an alternative set of keys to access that data is called a secondary database. In a secondary database, the key is the alternative index, and the data corresponds to a primary record's key [41] .

In CASH2 implementation, we created primary databases according to the database 
schema in Figure 5.7. The primary key of each table is stored in the key part of the record in corresponding primary database. Data is stored in the data part of the record in corresponding primary database.

Besides the primary database, we designed and implemented a number of secondary databases to provide fast lookup and rapid access to the data we want to retrieve.

CategoryClassification In this table, we created several secondary databases with their corresponding keys as shown below:

\section{Cid}

The secondary database is used in computing category path and used for detecting cycles operations.

\section{ParentCid}

The secondary database is used in computing category path, and for displaying category and file classifications information.

\section{Cname}

The secondary database is used in matching path operation, and for displaying category and file classifications information.

\section{ParentCid + Cname}

The secondary database is used in matching path operation.

\section{Cid + Cname}

The secondary database is used in declassify a category from its parent category.

\section{ParentCid + Cid}

The secondary database is used in displaying category classifications information. 
FileClassification In this table, we created several secondary databases with their corresponding key shown below:

\section{Cid}

The secondary database is used in displaying file classification information.

\section{Fdev + Fino}

The secondary database is used in displaying the physical file information.

\section{Fname + Fdev + Fino + Dino}

The secondary database is used in removing file classification operations.

\section{Fname}

The secondary database is used in displaying file classification information.

\section{Cid + Fname}

The secondary database is used in displaying file classification information.

\subsection{Solutions to Existing Problems in Directory Hierarchy}

In Chapter 2, we described the existing problems in a directory hierarchy found in UNIX/Linux file systems. Henceforth, we will summarize how CASH2 is able to provide better solutions.

1. Problem: The nature of file organization determines that files are more likely to have several different classifications, however a directory hierarchy can only have a single classification for them.

Solution: CASH2 is based on multiple hierarchical categorization structure, which allows a file to be classified to more than one category, and each category may also have multiple parent categories. 
2. Problem: In a directory hierarchy, files have to be placed under the same directory, otherwise, their relationship is lost.

Solution: CASH2 is able to bring back the relationship between files under different directories by classifying them together. For example, "MPlayer" is a popular movie player developed for UNIX/Linux machines. After users have installed the package, its files are scattered under many different locations in different file systems, e.g., executable file mplayer is under /usr/bin, configuration files mplayer.conf and menu.conf, etc., are under /etc, and other sharable files between different users such as mplayer.desktop, mplayer.png, mplayer.l.gz and mplayer.html, etc, are under / usr/ share. In CASH2, users can bring back the relationships between these files by classifying them (using the command classify) into category $\mathbf{1}$ (with category name MPlayer), and further refining files into child categories $\mathbf{2}$ to $\mathbf{1 0}$ according to their wishes. The example is shown in Figure 6.21. After the classifying operation, users can issue the command Iscat $-\mathrm{R}$ MP layer to retrieve all the related information at once, or issue the rmcat -c MP layer to remove all the related information according to their wishes.

3. Problem: A directory hierarchy is a static organization scheme. When retrieving information, this static organization becomes restrictive. Users must remember the ordered sequence of directories they have used to organize the file when attempting to retrieve it by location.

Solution: In CASH2, every category name and logical file name in the multiple hierarchical categorization structure is indexed. Users are not bounded to the ordered sequence of categories during information retrieval. They only need to enter a category name or logical file name, and the system will search for it and display the result.

4. Problem: If users forget the location of files in a directory hierarchy, they will have 


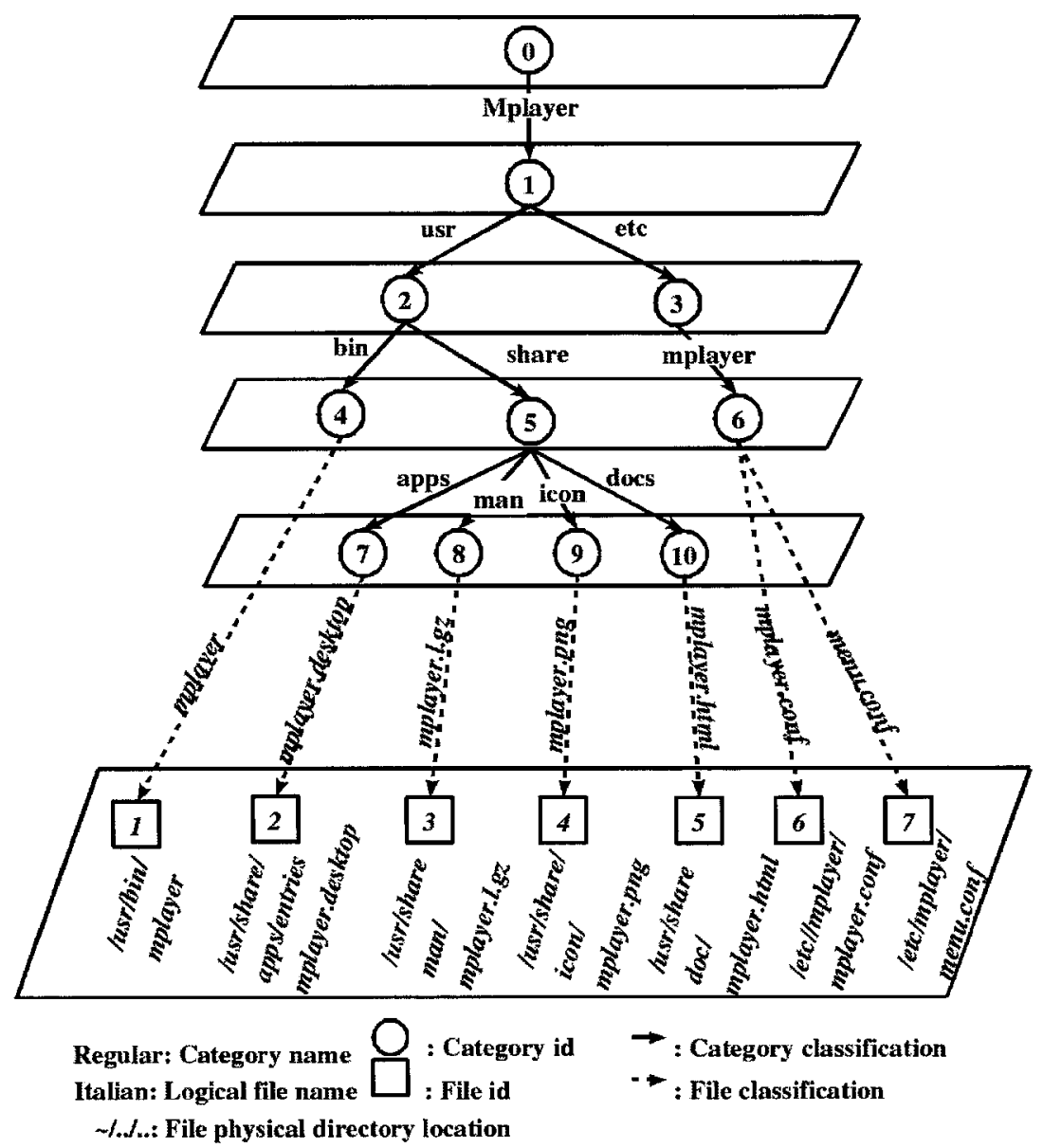

Figure 6.21: Example of Solution to Files with Lost Relationships 
to traverse the directory tree to find their files of interest.

Solution: Category names and logical file names in CASH 2 are indexed. Retrieving categories or files is done by indexed search instead of tree traversal. As a result, the search performance is improved significantly.

5. Problem: In the directory hierarchy, hard links cannot cross file system boundaries.

Solution: In CASH2, the File table has two attributes:

Fdev: Device number of the file, which represents the file system information where a file is located.

Fino: Inode number of the file, which uniquely identifies the file in the specified file system.

By saving these two attributes information, $\mathrm{CASH} 2$ is able to classify files in different file systems.

6. Problem: In the directory hierarchy, a symbolic link is a data file that contains the name of the file it is linked to, therefore, the connection between them is weak. Under the circumstance that the linked file is moved, renamed or deleted, the symbolic link would be left "dangling" and does not work properly anymore. In other words, symbolic links are poorly managed unless users manually maintain them.

Solution: In CASH2, the relationship between categories is a strong relationship as a result of the fact that once users delete a category by issuing the command rmcat $-\mathrm{c}$, all the relationships connected to it will be removed as well. Users are free from maintaining the category hierarchy.

7. Problem: UNIX/Linux system provides one command $\mathrm{rm}$ for both physical file deletion and link deletions. The system will delete the file without warning if the link is the last pointer to it, even through the users meant to delete a link and keep the file.

Solution: CASH2 provides two different utility commands to separate the operations 
of removing real files from removing links. The command rmcat $-\mathrm{f}$ is responsible for removing physical files, and the command decat $-f$ is responsible for declassifying a file from its specified category. Therefore, CASH2 system ensures that files will not be mistakenly removed.

8. Problem: In some cases a search in the directory hierarchy cannot be done by one tree traversal. Additional logical operations need to be applied to all the tree traversal results as well. However, there is no convenient way for users to work this out. Users must learn to write a shell script, looping through each tree traversals while calculating the result.

Solution: $\mathrm{CASH} 2$ provides a utility command $1 \mathrm{scat}-\mathrm{F}$ to operate on different file search results. It enables logical operations on sets of file information obtained from different sub-DAGs in the multiple hierarchical categorization structure. the logical operations includes AND, OR and NOT, which not only enriches the search features in $\mathrm{CASH} 2$, but also brings more convenience for file retrieval.

9. Problem: Information about all the links of a given directory or file in the directory hierarchy would help users to find related directories and files of interest within them. But due to the fact that the directory hierarchy can only provide top-to-bottom search, and not the other way around, there is no convenient way to achieve this task. Users have to again write a shell script to get the result.

Solution: CASH2 enables bidirectional searches (i.e., both top-to-bottom search and bottom-to-top search), therefore, the system is able to construct a full path information from a certain category or file. Users can issue a command Iscat $-\mathrm{si}$ to display all the path information of a target category, and Iscat $-\mathrm{f}$ or Iscat $-F$ to display files with all its paths information. Both commands provides a better and convenient way for users to manage their categories or files, and browser for more information under related categories. 


\subsection{Search-based Performance Comparison}

As we mentioned in previous chapters, the directory hierarchy in UNIX/Linux file systems applies tree traversals to locate a certain directory or file, while In CASH2, we save the information of files and categories in a database and apply the B-tree index for searching information in the database. Theoretically, B tree indexing provides a fast lookup for information, but, because of other overhead, such as the overhead caused by the fact that $\mathrm{CASH} 2$ is built on top the file systems ( not as part of the operating system, etc.), the final performance may suffer from the additional overhead.

To create a testing environment of our experiments, we mapped the UNIX file systems into CASH2. For simplicity, we only mapped real directories and files (not links) into the databases in CASH2. After mapping, the table File has 506,215 records, the table Directory has 41,840 records, the table Category has 41,840 records, the table CategoryClassification has 41,840 records, and the table FileClassification has 506,215 records.

\subsubsection{Experiment A: Directory/Category and File Search}

In this experiment, our intent is to find out the search performance between UNIX and $\mathrm{CASH} 2$ when the location of files is given.

The UNIX command we use is

Is -RI directory path

which means we list all the directories and files under the given directory path recursively in long form.

The CASH 2 command we use is

lscat -RI category path

which means to list all the categories and files under the given category path recursively in long form.

The searching result is shown in Figure 6.22. We see that for queries with a low file match, 
CASH2 is around $25 \%$ to $50 \%$ slower than UNIX. This is because CASH2, as an add-on application, has to interact with UNIX file system first before conducting operations in the operating system. However, as the number of files that match the query increases, the performance differences reduce as the search in UNIX suffers the overhead of swapping inode information into memory as well.

\begin{tabular}{|c|c|c|c|}
\hline $\begin{array}{c}\text { Number of file } \\
\text { that matched }\end{array}$ & $\begin{array}{c}\text { Number of } \\
\text { directory/category } \\
\text { that matched }\end{array}$ & $\begin{array}{c}\text { UNIX command: } \\
\text { Is -RI directory } \\
\text { Time (sec.) }\end{array}$ & $\begin{array}{c}\text { CASH2 command: } \\
\text { Iscat -RI category } \\
\text { Time (sec.) }\end{array}$ \\
\hline 506215 & 41840 & 162.884 & 162.013 \\
\hline 59200 & 3226 & 13.090 & 16.425 \\
\hline 48421 & 2321 & 11.028 & 15.111 \\
\hline 958 & 30 & 0.190 & 0.238 \\
\hline
\end{tabular}

Figure 6.22: Experiment A: Directory/Category and File Search

\subsubsection{Experiment B: Directory/Category Path Search}

In this experiment, our intent is to find out the performance of searching directory/category path information between UNIX and CASH2.

The UNIX command we use is

find directory path -type d

which means to list all the directory path information starting at the input directory.

The CASH2 command we use is

lscat -P category path

which means to list all the category path starting at the input category.

The search result is shown in Figure 6.23. We see that for queries with a low directory/category path match, CASH2 is around $50 \%$ to $80 \%$ faster than UNIX. This is because directory entry in UNIX is searched sequentially, while in CASH2, categories are all B-tree 
indexed, which improves the search significantly. As the number of directory/categories increases and it gets closer to its full volume in the system, the performance differences reduce as a B-tree search begins to approach the performance of a sequential search.

\begin{tabular}{|c|c|c|}
\hline $\begin{array}{c}\text { Number of } \\
\text { directory/category path } \\
\text { that matched }\end{array}$ & $\begin{array}{c}\text { UNIX Command: } \\
\text { find directory -type d } \\
\text { Time (sec.) }\end{array}$ & $\begin{array}{c}\text { CASH2 Command: } \\
\text { Iscat -P category } \\
\text { Time (sec.) }\end{array}$ \\
\hline 34729 & 100.496 & 94.496 \\
\hline 9859 & 27.519 & 27.490 \\
\hline 2284 & 5.024 & 1.451 \\
\hline 1740 & 2.912 & 0.590 \\
\hline
\end{tabular}

Figure 6.23: Experiment B: Directory/Category Path Search

\subsubsection{Experiment C: File Search}

In this experiment, our intent is to find out the performance of file search between UNIX and $\mathrm{CASH} 2$.

The UNIX command is

\section{find / -name "file name"'}

which means we list all the files with their name matches file name.

The CASH2 command is

lscat $-F$ ' 'file name'"

which means we list all the files in $\mathrm{CASH} 2$ with their logical name matches file name.

The search result is shown in Figure 6.24. CASH2 is around $21 \%$ to $50 \%$ faster than UNIX. This is because in UNIX, too much redundant time is spent on traversing the tree structure hierarchy to find the file, while in CASH2, th file name is indexed in table FileClassification, which result in better search performance. 


\begin{tabular}{|c|c|c|}
\hline $\begin{array}{c}\text { Number of } \\
\text { Files } \\
\text { that matched }\end{array}$ & $\begin{array}{c}\text { UNIX Command: } \\
\text { find } / \text {-name } \\
\text { Time (sec.) }\end{array}$ & $\begin{array}{c}\text { CASH2 Command: } \\
\text { Iscat }-F \\
\text { Time (sec.) }\end{array}$ \\
\hline 506215 & 111.985 & 81.230 \\
\hline 23477 & 106.485 & 67.827 \\
\hline 14381 & 96.482 & 53.267 \\
\hline 5875 & 95.289 & 50.198 \\
\hline
\end{tabular}

Figure 6.24: Experiment C: File Search

\subsubsection{Experiment D: Category-based Search by lscat -F}

In this experiment, our intent is to find out the search performance between UNIX and $\mathrm{CASH} 2$ when the location of a target file is unknown. For example, we have a file name "TestSampleBodyTag.java", and its physical location is under the directory path "/usr/ JBuilder2005/ thirdparty/ jakarta-cactus-13-1.6.1/ src/ integration/ maven/ samples/ servlet/ src/ test-cactus/ org/ apache/ maven/ cactus/ sample". We assume that a user forgets this long path information, however they may remember some piece of information about the file, such as the file is related to "src", "servlet", "sample", "apache" and "JBuilder2005". In UNIX, without knowing the exact location of a file, we can only search the whole file system for it.

The command we use is

find / -name 'TestSampleBodyTag.java',

In $\mathrm{CASH} 2$, because the searches are not restricted with the category path that a file is classified under, we have more choices to find the file by using lscat $-\mathrm{F}$.

Examples are as follows:

1. Iscat $-\mathrm{F}$ Test SampleBodyTag.java

This command will search the table FileClassification by the file name to find the file. 
2. Iscat $-\mathrm{F}$ sample/Test SampleBodyTag.java

This command will search the category name "sample" and locate the file.

3. lscat $-\mathrm{F}$ servlet/src/TestSampleBodyTag.java

This command will search the category path "servlet/src" and locate the file.

4. Iscat $-\mathrm{F}$ apache/Test SampleBodyTag.java

This command will search the category name "apache" and locate the file.

5. Iscat $-F$ JBuilder2005/TestSampleBodyTag.java

This command will search the category name "JBuilder2005" and locate the file.

The search result is shown in Figure 6.25. We see that

lscat $-F$ Test SampleBodyTag.java

is about 370 times faster than the UNIX find command.

The rest of the commands are around 30 to 50 times faster than UNIX find command, because more time was spent on searching the category name or path information of the target file.

\begin{tabular}{|c|c|c|}
\cline { 3 - 3 } \multicolumn{2}{c|}{} & Time (sec.) \\
\hline \multirow{3}{*}{ Command in UNIX } & find / -name TestSampleBodyTag.java & 111.428 \\
\hline & Iscat -F TestSampleBodyTag.java & 0.300 \\
\cline { 2 - 3 } & Iscat -F sample/TestSampleBodyTag.java & 2.072 \\
\cline { 2 - 3 } & Iscat -F servlet/src/TestSampleBodyTag.java & 1.530 \\
\cline { 2 - 3 } & Iscat -F apache/TestSampleBodyTag.java & 2.622 \\
\cline { 2 - 3 } & Iscat -F Jbuilder2005/TestSampleBodyTag.java & 3.222 \\
\hline
\end{tabular}

Figure 6.25: Experiment D: Category-based Search by lscat $-F$ 


\subsubsection{Summary}

To summarize, the CASH2 system has overhead compared to UNIX/Linux file systems. However, the performance of CASH2 is very good when considering the fact that it combines index and multiple hierarchical categorization structure for searching, and allow users to organize their information is a more convenient and better way. 


\section{Chapter 7}

\section{CONCLUSION REMARKS AND FUTURE WORK}

CASH2 is designed to be a novel file management tool for UNIX/Linux systems. Based on a multiple hierarchical categorization structure, which allows a file to be classified to more than one category, and a category could also have multiple parent categories, CASH2 overcomes some of the drawbacks of the existing directory hierarchy and provides a more flexible way for users to manage their categories and files. CASH2 made significant progresses on its predecessor. Below we summarize our contributions:

1. We studied the existing solutions related to file management and search technologies, such as semantic file systems, desktop search tools and category-based applications. We analyzed them briefly for their pros and cons in order to improve our research in a better way. This achieved objective (1) of this thesis.

2. We formalized the basic concepts in CASH2 design, such as Category, Category and File Classification, and Multiple hierarchical categorization structure, etc. This achieved objective (2) of this thesis.

3. We summarized the drawbacks of the existing directory hierarchy, and explained 
the improvements to solve these problems in CASH2 accordingly. This achieved objective (3) of this thesis.

4. We explained our modeling methodology, Entity Relationship Modeling, and our design work based on that. This achieved objective (4) of this thesis.

5. We studied different database engines and analyzed their pros and cons in regards to the requirements of CASH2 applications. We replaced the MySQL database engine with Berkley DB database engine to fit our needs. This achieved objective (5) of this thesis.

6. We implemented the CASH2 implementation, illustrated our algorithms, and achieved our objective (6) of this thesis.

7. We extended the command design with more features, such as Iscat -i, 1scat $-F$, Iscat $-p$, Iscat $-P$, and cdcat $-p$. This achieved objective (7) of this thesis.

8. We conducted search-based experiments, provided performance results as system measurement, and achieved objective (8) of this thesis.

Our work on CASH2, however, is far from complete. CASH2 has some limitations and there is still much research and implementation work yet to be done. Some of the work is mentioned below:

1. Category access control in CASH2 is not implemented as it is a separate research field, and this requires more study to be done in this aspect.

2. In CASH2, we did not provide a mechanism to let other applications plug in easily. More works need to be done in this aspect. 
3. More study can be done to provide a more visually user-friendly interactive interface for CASH2 applications.

4. More developing tools, such as OpenGL, 3Dfile, etc, can be considered as other approaches to implement the multiple hierarchical categorization structure in UNIX/Linux machine.

5. More research work can be done to realize the design ideas in CASH2 by modifying the UNIX/Linux kernels in operating system design. 


\section{Chapter 8}

\section{APPENDIX: BRIEF DESCRIPTIONS OF USER COMMANDS}

Command: Category

- Description: Switching the current prompt to category prompt.

Command: Directory

- Description: Switching the current prompt to directory prompt.

Command: load directory

- Description: Mapping the directories and files in UNIX/Linux file systems into CASH2.

Command: lscat [option] [target]

- Description: Displaying the information of categories, category classification, files, or file classification. 


\section{- Options:}

none: List the content of the target category, including all the category classification and file classification information with the target as the parent category.

-w: Displays the target category (a wild card matching on category name is enabled) information in the system.

-1 : Displays in long form.

$-\mathrm{R}$ : Recursively displays all category and file information in the sub-DAG rooted at the target category.

$-s$ : Displays the self-information of the target category.

- $i$ : Displays the alias information of the target category.

$-\mathrm{P}$ : Displays all the path information in the sub-DAG rooted at the target category.

$-\mathrm{C}$ : Displays all the path information from root to the target category.

$-\mathrm{p}$ : Gives uses options to choose a parent category of the target category.

$-\mathrm{f}$ : Displays the target file information under its specified category.

$-F$ : Displays the target file information under its predecessor category.

\section{Command: cdcat [option] [target]}

- Description: Changing the working category to target.

\section{- Options:}

none: Change into the child category of the current working category.

$-u$ : Forces to search the target category name or path information in the whole system.

$-\mathrm{p}$ : Gives parent category options of the target category for users to choose one and work under it.

Command: mkcat [option] [search path] [category name or path] 
- Description: Making new categories.

\section{- Options:}

none: Creates new category under the current working category.

$-\mathrm{p}$ : Search path is presented, under which new category is created.

\section{- Examples:}

mkcat newcat1 newcat2 newcat3/newcat4

mkcat -p search-path-cat1/cat2 newcat 1 newcat 2 newcat $3 /$ newcat 4

Command: classify [option] [category name or path] [source] [new category name or path]

- Description: Classifying files and categories.

\section{- Options:}

none: source is a category.

$-\mathrm{f}$ : source is a file or directory in directory format.

${ }_{-\mathrm{C}}$ : source is a logical file name in category format.

\section{Command: Incat}

- Syntax:

lncat [ Target]

or

lncat [Target] [alias name]

- Description: Creating aliases for a category classification.

- Examples:

lncat some/name \# create ./name point to /some/name

lncat some/name myname \# create ./myname point to /some/name 


\section{Command: rmcat [option] [target]}

- Description: Removing categories and files.

\section{- Options:}

none: target is a category name or path.

$-f$ : target is a logical file name in category format, and all the categories in the subDAG rooted at target will be removed.

$-\mathrm{k}$ : target is a category name or path, and all the categories in the sub-DAG rooted at target that do not have aliases that do not belong to the sub-DAG will be removed. -a: Clear all the data in the system.

\section{Command: decat [option] [category name or path] [target]}

- Description: Removing file classification and category classification.

\section{- Options:}

none: target is a category name.

$-\mathrm{f}$ : target is a logical file name.

\section{Command: edcat [option] source text}

- Description: Editing a category or file classification description

\section{- Options:}

none: source is a category name, text is a short description of the category classification.

$-\mathrm{f}$ : source is a file logical name, text is a short description of the file classification. 
- Examples:

edcat catname desc

edcat -f filename desc

Command: rename originalname newname

- Description: Renaming an existing category or logical file name.

\section{- Options:}

none: originalname is a category name.

-f: originalname is a logical file logical name. 


\section{Bibliography}

[1] John R. Doucer, William J. Bolosky. A large-scale study of file-system contents. ACM SIGMETRICS Conference on Measurement and Modeling of Computer Systems(Atlanta, GA, 1-4 May 1999). Published as ACM SIGMETRICS Performance Evaluation Review, 27(1):59-70. ACM Press, 1999.

[2] Craig A. N. Soules, Gregory R. Ganger. Toward automatic context-based attribute assignment for semantic file systems. Carnegie Mellon University Parallel Data Lab Technical Report CMU-PDL-04-105, June 2004.

[3] William Stallings. Operating Systems - Internals and Design Principles, Fifth Edition. Prentice Hall, 2005

[4] Staelin, C. and Garcia-Molina, H. File system design using large memories. Information Technology, 1990. 'Next Decade in Information Technology', Proceedings of the 5th Jerusalem Conference on 22-25 Oct. 1990 Pages: 11 - 21

[5] Florin Isaila. An Overview of File System Architectures. Lecture Notes in Computer Science, Springer-Verlag GmbH, Volume 2625 in 2003, Pages: 273 - 289

[6] Idaho State University. A Basic UNIX Tutorial. www.isu.edu/ departments/ comcom/ unix/ workshop/ fstour.html, Jan. 9, 1996, Idaho State University 
[7] Unix Workstation Support Group (UWSG), Indiana University. Unix System Administration Independent Learning. www.uwsg.iu.edu/usail/peripherals/disks/filesystems

[8] Red Hat, Inc. Red Hat Linux 9: Red Hat Linux Reference Guide, Chapter 3. File System Structure. 2004 Red Hat, Inc.

[9] M. Lansdale. The Psychology of Personal Information Management. 1998, Applied Ergonomics 19(1), page 55 - 66

[10] UNIX man pages: $\ln () .2002$, Free Software Foundation, Inc.

[11] Udi Manber and Sun Wu. GLIMPSE: a tool to search through entire file systems. Winter USENIX Technical Conference (San Francisco, CA, January 1994), pages 23 - 32. USENIX Association, 1994

[12] Dennis Quan, Karun Bakshi, David Huynh and David R. Karger. User Interfaces for Supporting Multiple Categorization. INTERACT September 2003.

[13] Lan Yang. CASH: A Category - based File Management System. Master Thesis, Computer Science, Carleton University, April, 2005.

[14] Raghu Ramakrishnan and Johannes Gehrke. Database Management Systems, Third Edition. p738, August 14, 2002

[15] Michael Erdmann. Formal Concept Analysis to Learn from the Sisyphus-Ill Material. Proceedings of the 11th Knowledge Acquisition for Know,ledge-bosed Systems Workshop (KSW's Ya), Alberta, Canada, Apr. 1998, pp. SIS-2.

[16] Chen, P. The Entity Relationship Model - Towards a Unified View of Data. ACM Transactions on Database System 1, no.1 (1976): 9 - 36

[17] Booch, G. Object-Oriented Design with Applications. 3nd edition. Addison-Wesley, 2004 
[18] Coad, P., and E.Yourdon. Object-Oriented Analysis. Englewood Cliffs, N.J.: Prentice Hall, 1991

[19] Rumbaugh, J., J. M. Blaha, W. Premerlani, F. Eddy, and W. Lorenson. ObjectOriented Modeling and Design with UML. Prentice Hall, November 29, 2004

[20] Shlaer, S., and S. Mellor. Object Oriented Systems Analysis. Englewood Cliffs, N.J.: Prentice Hall, 1988

[21] Barreau, D. and Nardi, B.A. Finding and reminding: File organization from the desktop. SIGCHI Bull 39-43 July, 1995

[22] Ellie Quigley. UNIX Shells by Example Fourth Edition. Chapter 1, Prentice Hall, July 12,1999

[23] David K. Gifford, Pierre Jouvelot, Mark A. Sheldon, and James W. O’Toole, Jr. Semantic File Systems. 13th ACM Symposium on Operating Systems Principles, October 1991

[24] Dominic Giampaolo. Practical File System Design with the Be File System. 1999 Morgan Kaufmann Publishers, Inc.

[25] Burra Gopal and Udi Manber. Integrating Content-Based Access Mechanisms with Hierarchical File Systems. Symposium on Operating Systems Design and Implementation, page 265-278, ACM, 1999

[26] Hui-I Hsiao and Inderpal Narang. DLFM: A Transactional Resource Manager. May 2000, ACM SIGMOD international conference on Management of data SIGMOD '00, Volume 29 Issue 2

[27] Google Desktop, desktop.google.com. May, 2006

[28] Yahoo Desktop, desktop.yahoo.com. May, 2006 
[29] MSN Toolbar, toolbar.msn.com, May, 2006

[30] Paula MacKinnon Looking for Mr. Goodtoolbar. 2006 Business Information Group. A member of the esourceNetwork.

[31] Verity Inc. Verity Collaborative Classifier Overview. www.verity.com Verity Inc.

[32] Inktomi's Content Classification Engine Module, searchmarketing.yahoo.com

[33] Inxight's Categorizer, www.inxight.com. May, 2006

[34] Mohomine's Mohn Classifier, www.kofax.com. May, 2006

[35] Autonomy Corporation plc., www.autonomy.com. May, 2006

[36] Entrieva Inc., www.entrieva.com. May, 2006

[37] Bernard Chester. Auto Categorization and Records Management. March/April 2004, E-Doc Magazine

[38] Tom Reamy. Cyborg Categorization Part 1 - The Salvation of Search. Intranet Professional, Jan/Feb 2002 Issue

[39] Bibliographix, www.bibliographix.com. May, 2006

[40] A wiki-based open content dictionary. en.wiktionary.org, May, 2006

[41] SLEEPYCAT SOFTWARE. Berkeley DB Reference Guide. Sleepcat Software, Inc. 1996-2004.

[42] Michael A. Olson, Keith Bostic, and Margo Seltzer. Berkeley DB. Proceedings of the FREENIX Track: 1999 USENIX Annual Technical Conference, Monterey, California, USA, June 6th, 1999 
[43] SLEEPYCAT SOFTWARE. Getting Started with Berkeley DB for C. SIGCHI Bull 22 September 2004.

[44] Comer, D. The Ubiquitous B-tree. ACM Computing Surveys Volume 11, number 2, June 1979

[45] Litwin, W. A new tool for file and table addressing. Proceedings of the Sixth International Conference on Very Large Data Bases IEEE Computer Society, 1980, p212 $-223$

[46] Stonebraker, M., Stettner, H., Kalash, J.,Guttman, A., and Lynn, N. Document Processing in a Relational Database System. Memorandum No. UCB/ERL M82/32, University of California at Berkeley, Berkeley, CA, May 1982.

[47] FireBird Relational Database. www.firebirdsql.org. 2000-2005.

[48] db.* User's Guide, www.ittia.com/dbstar. 2000-2005.

[49] SQLite, www.sqlite.org. September, 2005.

[50] Apache Derby Project, db.apache.org/derby. Feburary, 2006.

[51] MySQL 5.0 Reference Manual, dev.mysql.com/ doc/ refman/ 5.0/ en/ libmysqldoverview.html. 1995-2006 MySQL AB. 